\title{
A chemistry and cooling module for the NIRVANA code
}

\author{
U. Ziegler
}

\author{
Leibniz-Institut für Astrophysik Potsdam, An der Sternwarte 16, 14482 Potsdam, Germany \\ e-mail: uziegler@aip.de
}

Received 6 August 2018 / Accepted 3 October 2018

\begin{abstract}
Context. Gas cooling and other thermal processes in the interstellar medium are intimately related to its chemical evolution. To accurately model chemical processes in large-scale gas-dynamical simulations the usage of existing sophisticated astrochemical networks is presently impossible due to prohibitive computational costs. A viable way to deal with the problem is by the design of reduced chemical networks that satisfactorily reproduce the most important features of the more elaborate networks.

Aims. A chemistry and cooling module for the interstellar medium is developed that is realistic for temperatures $T \gtrsim 50 \mathrm{~K}$ and for densities up to $n \approx 10^{10} \mathrm{~m}^{-3}$ at the limit of non-ionizing and non-dissociating background radiation. The module is incorporated into the multiphysics, adaptive-grid code NIRVANA and aims at improving gas-dynamical simulations by explicitly following nonequilibrium chemistry and gas cooling.

Methods. The presented chemical network covers 121 species and 426 reactions. It includes a fully-fledged ionization subnetwork for the ten elements $\mathrm{H}, \mathrm{D}, \mathrm{He}, \mathrm{C}, \mathrm{N}, \mathrm{O}, \mathrm{Mg}, \mathrm{Ne}, \mathrm{Si}$, and $\mathrm{Fe}$, chemical schemes for the formation and destruction of the important molecular coolants $\mathrm{H}_{2}, \mathrm{H}_{2} \mathrm{O}, \mathrm{CO}$, and $\mathrm{OH}$, a model for dust-catalytic reactions and cosmic ray effects. Metal line cooling was computed from first principles by solving for the energy level population for each ion. Atomic data was adopted from the latest version of the Chianti database. The treatment of rotovibrational line cooling from molecules was based on various up-to-date literature sources. Results. The implementation has been validated by performing both equilibrium and non-equilibrium (time-dependent) computations. The equilibrium results overall confirm the temperature dependence of chemical abundances and the gas cooling rate that has been found in similar studies. In particular, the ionization structure in the high-temperature regime, at $T \gtrsim 2 \times 10^{4} \mathrm{~K}$, excellently agrees with literature results. In the non-equilibrium calculations, the occurrence of ionization lags are prominent and distinguishes the resulting non-equilibrium cooling from equilibrium cooling. In the low-temperature regime, at $T \lesssim 2 \times 10^{4} \mathrm{~K}$, the non-equilibrium cooling rate can be enhanced by up to two orders of magnitude compared to the equilibrium value.

Conclusions. The NIRVANA chemistry and cooling module has been successfully tested against literature results. The underlying chemical network is best compared to recently developed networks in the limit of no radiation with differences appearing in the treatment of dust, cosmic ray heating, and in the choice of reaction coefficients. The gas cooling experiments indicate that a reduction of the present network size seems possible under certain conditions by skipping the elements $\mathrm{N}$ and $\mathrm{Mg}$, which turn out to be less important coolants compared to the conglomerate of $\mathrm{C}, \mathrm{O}, \mathrm{Si}$, and $\mathrm{Fe}$ coolants.
\end{abstract}

Key words. astrochemistry - magnetohydrodynamics (MHD) - methods: numerical

\section{Introduction}

The composition of gas in the interstellar medium (ISM) can be subject to changes via chemical processes of great diversity. Knowledge of the underlying chemistry is potentially important because species abundances regulate the thermal properties of the gas. Thermal processes like radiation cooling can have, in turn, a strong impact on the gas dynamics. When the dynamics and thermal effects of gas get strongly coupled with its chemical changes, non-equilibrium behavior can occur. The assumption of instantaneous chemical equilibrium then becomes disputable and a time-dependent treatment of chemical processes seems imperative. For example, ionization equilibrium is quite assumed. This assumption breaks down, however, when gas cooling occurs more rapidly than ion recombination. In this case, ionization lags result, which lead to an enhancement or reduction of radiation cooling depending on the temperature regime (see, e.g., Gnat \& Sternberg 2007; Oppenheimer \& Schaye 2013a). In general, cooling rates can become rather inaccurate if chemical equilibrium is predisposed. The assumption of instantaneous chemical equilibrium can also break down in a flow that is sufficiently turbulent. If the eddy turnover time is short enough, physical conditions experienced by a gas parcel may change before chemical equilibrium can be established (Gray et al. 2015). Furthermore, in the presence of an ionizing radiation field that varies on a timescale shorter than the recombination time, the ionization balance is expected to be out of equilibrium and non-equilibrium effects come into play (Oppenheimer \& Schaye 2013b).

Atomic and molecular line cooling is central to many ISM problems. For a reasonable modeling of non-equilibrium cooling efficiencies, the chemical state of the gas must be known. This requires us to solve a chemical network in accordance with the gas-dynamical equations. The most comprehensive chemical networks for the ISM are found in astrochemical databases like the UMIST database (Le Teuff et al. 2000) or the Kinetic Database for Astrochemistry (KIDA, Wakelam et al. 2012), which contain thousands of reactions between hundreds of species. Combining such sophisticated networks with largescale dynamical simulations is far out of reach, however, due to prohibitive computational costs. One must therefore resort to reduced networks that are optimally constructed in such a way that relevant features of larger networks are subsumed. Of particular interest in this respect is to identify the most important 
coolants and to capture those reactions that have a significant influence on the abundance of those coolants. The selection of a proper subset of reactions from a larger network is also guided by the temperature and density range of the problem or diagnostic purposes. The size of a chemical network that can be tackled within gas-dynamical simulations is severely limited in any case by the available computing power to solve its rate equations.

An example of a reduced network is that of Nelson \& Langer (1999) focusing on the formation of carbon monoxide, CO. In this simplified network, the effects of intermediate hydrocarbon species like $\mathrm{CH}$ or $\mathrm{CH}_{2}$ and (hydro-)oxygen species like $\mathrm{OH}$ and $\mathrm{H}_{2} \mathrm{O}$ are consolidated in the form of (artificial) composite species $\mathrm{CH}_{x}$ and $\mathrm{OH}_{x}$. This greatly reduces the network complexity at the expense of not tracking distributions of such intermediate species. The benefit of the Nelson \& Langer (1999) approach has been proved by Glover \& Clark (2012) who compared different networks for modeling CO chemistry in molecular clouds. Another example of a useful simplification is the chemical network of Glover \& Jappsen (2007) designed to study low-density, low-temperature gas with low metal content. This network consists of 74 reactions that evolve 18 species. It includes the molecular coolants $\mathrm{H}_{2}$ and $\mathrm{HD}$ but ignores other molecular coolants like $\mathrm{CO}$ and $\mathrm{H}_{2} \mathrm{O}$ (relevant at higher densities), and it neglects higher ionization states of metals like $\mathrm{C}, \mathrm{O}$, and $\mathrm{Si}$ (relevant at higher temperatures). This network has been later extended by adding carbon and oxygen molecular chemistry in the work of Glover et al. (2010; GFM10). Recently, a chemistry and cooling model for the diffuse ISM has been constructed by Richings et al. (2014; RSO14) taking the GFM10 chemical network as a basis to represent the low-temperature regime $\left(T \lesssim 2 \times 10^{4} \mathrm{~K}\right.$ ), and combining it with the ionization and cooling model of Oppenheimer $\&$ Schaye (2013a) appropriate in the high-temperature regime $\left(T \gtrsim 2 \times 10^{4} \mathrm{~K}\right)$.

In an earlier paper (Ziegler 2016), a chemical reaction network solver for the magneto-hydrodynamics code NIRVANA (Ziegler 2008, 2011, 2012) was developed. For the solution of the ordinary differential equation (ODE) system resulting from the rate equations of chemical kinetics and thermal processes, a fourth-order stiff integrator of Rosenbrock-Wanner type was implemented. The thermo-chemistry solver was coupled to the magneto-hydrodynamics scheme in a Strang-type operator-splitting fashion. In conjunction with this development, a very rudimentary chemical model for the ISM was introduced describing $\mathrm{H} / \mathrm{He}$ ionization and gas-phase $\mathrm{H}_{2}$ formation through the well-studied $\mathrm{H}^{-} / \mathrm{H}_{2}^{+}$channel.

In this paper a substantially extended NIRVANA chemistry and cooling module $\left(\mathrm{NC}^{2} \mathrm{M}\right)$ is presented inspired by the work of RSO14. To begin with, the $\mathrm{NC}^{2} \mathrm{M}$ comes with two simplifications compared to RSO14, which significantly reduce the network size. First, the less abundant elements $\mathrm{S}$ and $\mathrm{Ca}$ are neglected because they are minor coolants in interstellar gas. Second, any photoionizing background is ignored at the current stage of development. This allows us to cut down photochemical reactions and to dispense with the process of Auger ionization by energetic photons. The second assumption is a critical one and, to be valid in reality, requires the gas to be somehow shielded from the background ultraviolet radiation field of the ISM. This is the case, for instance, in the interior regions of dense clouds where chemical processes are controlled by gas-phase reactions, cosmic ray ionization, and by reactions on grain surfaces, and where photoionization and photodissociation effects play only a minor role. In such shielded regimes, which generally tend to be cold, the high-temperature chemistry included in the $\mathrm{NC}^{2} \mathrm{M}$ becomes non-essential and can be switched off as an option. Photochemical processes will be added in a future version of the $\mathrm{NC}^{2} \mathrm{M}$ to account for the effects of an ionizing background.

There are further differences to the work of RSO14. The RSO14 network does not consider deuterium chemistry. Following Omukai et al. (2005) and Glover \& Abel (2008), it may become non-negligible in dense, low-metallicity gas. Although the $\mathrm{NC}^{2} \mathrm{M}$ aims at applications for metal-enriched gases in the ISM, a mini-network of deuterium chemistry has been included as an option. Fine-structure metal line cooling is treated in the $\mathrm{NC}^{2} \mathrm{M}$ from first principles by solving the statistical equilibrium equation for the ion level populations, thereby making use of the Chianti atomic database (Del Zanna et al. 2015). In RSO14 ionby-ion cooling tables are applied that were precomputed with help of the spectral synthesis code Cloudy (Ferland et al. 2013). Additional minor differences affect the used kinetic- and thermal rate coefficients, the number of charge transfer reactions between metal species, details in the dust model, and the more elaborate treatment of cosmic-ray heating in the $\mathrm{NC}^{2} \mathrm{M}$.

\section{The chemical model}

Following Ziegler (2016), the chemical evolution extends the system of gas-dynamical equations by adding advection-reaction equations for the number densities $n_{s}$ of different species $X_{s}, s=$ $1 \ldots N_{s}$, of the form

$\partial_{\mathrm{t}} n_{s}+\nabla \cdot\left(n_{s} \mathbf{v}\right)=R_{s}=\sum_{r=1}^{N_{r}}\left(\beta_{s, r}-\alpha_{s, r}\right) k_{r} \prod_{\substack{l \\ X_{l} \in R(r)}} n_{l}^{\alpha_{l, r}}$,

where $\mathbf{v}$ is the flow field, $k_{r}$ are the kinetic rate coefficients for the chemical reactions, $r=1 \ldots N_{r}$, and $\alpha$ and $\beta$ are stoichiometric coefficients. The reaction source terms $R_{s}$ in Eq. (1) derive from the set of chemical reactions

$$
\sum_{X_{s} \in R(r)} \alpha_{s, r} X_{s} \stackrel{k_{r}}{\rightarrow} \sum_{\substack{S \\ X_{s} \in P(r)}} \beta_{s, r} X_{s}, \quad r=1 \ldots N_{r}
$$

between reactants $X_{s} \in R(r)$ leading to products $X_{s} \in P(r)$ with $R(r)$ and $P(r)$ being subsets of $\left\{X_{s}\right\}_{s=1}^{N_{s}}$.

The $\mathrm{NC}^{2} \mathrm{M}$ evolves 121 species. Referring to standard solar abundances as cataloged in Grevesse \& Sauval (1998), the nine most abundant elements $\mathrm{H}, \mathrm{He}, \mathrm{C}, \mathrm{N}, \mathrm{O}, \mathrm{Ne}, \mathrm{Mg}, \mathrm{Si}$, and $\mathrm{Fe}$ are considered. It is supplemented by deuterium, $\mathrm{D}$, which is believed to play a role in primordial or low-metallicity contexts. The total set of species consists of all ionization states of these elements, the negatively charged species $\mathrm{H}^{-}, \mathrm{C}^{-}$, and $\mathrm{O}^{-}$, and the molecules $\mathrm{H}_{2}, \mathrm{H}_{2}^{+}, \mathrm{H}_{3}^{+}, \mathrm{HD}, \mathrm{C}_{2}, \mathrm{O}_{2}, \mathrm{O}_{2}^{+}, \mathrm{CH}, \mathrm{CH}^{+}, \mathrm{CH}_{2}$, $\mathrm{CH}_{2}^{+}, \mathrm{CH}_{3}^{+}, \mathrm{OH}, \mathrm{OH}^{+}, \mathrm{CO}, \mathrm{CO}^{+}, \mathrm{H}_{2} \mathrm{O}, \mathrm{H}_{2} \mathrm{O}^{+}, \mathrm{H}_{3} \mathrm{O}^{+}, \mathrm{HCO}^{+}$, and $\mathrm{HOC}^{+}$. The chemical network has a total of 426 reactions, which is roughly half the size of the RSO14 network with 907 reactions. As already mentioned, the saving is due to the neglect of $\mathrm{S}$ and $\mathrm{Ca}$ and because of the absence of photochemical reactions. The $\mathrm{NC}^{2} \mathrm{M}$ includes gas-phase (atomic, ionic, molecular) reactions, cosmic ray reactions, and dust-assisted reactions. A compilation of all chemical reactions with references to sources for the rate coefficients is given in the appendix. The $\mathrm{NC}^{2} \mathrm{M}$ within its assessed limitations represents cooling gases with some fidelity for temperatures $T \gtrsim 50 \mathrm{~K}$ and up to number densities of $n \approx 10^{10} \mathrm{~m}^{-3}$, say. These thresholds in density and temperature are not to be understood as sharp values but rather as plausible estimates that remind us of the fact that at even higher 
density and lower temperatures, more complex molecules may be formed contributing to cooling, but which are not covered by the $\mathrm{NC}^{2} \mathrm{M}$. Despite such an upper density limit of validity, the $\mathrm{NC}^{2} \mathrm{M}$ optionally includes higher density cooling processes for the important $\mathrm{H}_{2}$ molecule, namely, a correction for optically thick line cooling and collision-induced emission cooling. The neglect of photoreactions in the $\mathrm{NC}^{2} \mathrm{M}$ would, strictly speaking, also require us to set a lower density limit above which the assumption of shielded gas is reasonable.

Technically, the $\mathrm{NC}^{2} \mathrm{M}$ is applicable (and will be applied) for any temperature and density. In doing so, however, one has to ensure that the implementation does not suffer from unphysical behavior or unboundedness of the rate coefficients in the asymptotic limits $T, n \rightarrow 0, \infty$. All rate coefficients are checked in this respect and, if necessary, are reasonably modified. An example is the rate coefficient from Kingdon \& Ferland (1996) for the charge transfer reaction $\mathrm{C}^{+}+\mathrm{H} \longrightarrow \mathrm{C}+\mathrm{H}^{+}$given by

$k=4.88 \times 10^{-22} \frac{\mathrm{m}^{3}}{\mathrm{~s}}\left(\frac{T}{10^{4} \mathrm{~K}}\right)^{3.25}\left[1-1.12 \exp \left(-\frac{0.21 T}{10^{4} \mathrm{~K}}\right)\right]$,

with specified temperature range $\left[5.5 \times 10^{3} \mathrm{~K}, 10^{5} \mathrm{~K}\right]$. Below $T \approx 5390 \mathrm{~K}$ the rate becomes negative, which is unphysical. Therefore, $k$ is set to zero below this threshold. Likewise, an upper floor is set at $T=10^{9} \mathrm{~K}$. Although $k$ becomes inaccurate for $T \gg 10^{5} \mathrm{~K}$, it will not have a large impact on the overall chemical evolution since the total reaction rate, $k n_{\mathrm{C}^{+}} n_{\mathrm{H}}$, for this process is negligible at such high temperatures owing to small number densities $n_{\mathrm{C}^{+}}$and $n_{\mathrm{H}}$. Generally, reaction rate coefficients are often not very precisely known. Uncertainties of $100 \%$ or even larger sometimes appear in the literature, which reduces confidence in the outcome of any reaction network (see Wakelam et al. 2005).

\subsection{Gas-phase reactions}

\subsubsection{Ionization, recombination, and charge transfer}

In calculating the ionization state of the gas, the chemical network of the $\mathrm{NC}^{2} \mathrm{M}$ includes the processes of collisional ionization $(\mathrm{CI})$,

$\mathrm{X}^{n+}+\mathrm{e}^{-} \rightarrow \mathrm{X}^{(n+1)+}+2 \mathrm{e}^{-}$,

radiative recombination $(\mathrm{RR})$,

$\mathrm{X}^{(n+1)+}+\mathrm{e}^{-} \rightarrow \mathrm{X}^{n+}(+h v)$

and di-electronic recombination (DR),

$\mathrm{X}^{(n+1)+}+\mathrm{e}^{-} \rightarrow \mathrm{X}^{n+, *} \rightarrow \mathrm{X}^{n+}(+h v)$,

for all elements $\mathrm{X}=\mathrm{H}, \mathrm{D}, \mathrm{He}, \mathrm{C}, \mathrm{N}, \mathrm{O}, \mathrm{Ne}, \mathrm{Mg}, \mathrm{Si}$, and $\mathrm{Fe}$. In the DR process, $\mathrm{X}^{*}$ denotes an intermediate excited state. Case A recombination is assumed for hydrogen and helium. CI, $\mathrm{RR}$, and DR rate coefficients for the lighter elements $\mathrm{H}, \mathrm{D}$, and He are taken from various sources as specified in Table A.1, reactions $1-6,350,351$. CI rate coefficients for metal species are described by the analytical fits in Voronov (1997), which encompass a large temperature range. RR and DR coefficients for almost all metal species rely on web-published ${ }^{1}$ atomic data and fitting functions based on detailed calculations with the code AUTOSTRUCTURE (Badnell 2006). Unfortunately, for higher isoelectronic sequences, which concerns all the ionization states of

1 http://amdpp.phys.strath.ac.uk/tamoc/DATA/ iron below $\mathrm{Fe}^{13+}$, no data is available. Therefore, for those ions the older RR coefficients from Shull \& van Steenberg (1982) are used, and DR coefficients are taken from Arnaud \& Raymond (1992).

The network also covers charge transfer (CT) interactions of various species with hydrogen and helium. Either a neutral $\mathrm{H}$ (or $\mathrm{He}$ ) atom transfers an electron to a species reducing its ionization state and $\mathrm{H}$ (or $\mathrm{He}$ ) becomes ionized or, inversely, an electron is transferred from a species, increasing its ionization state, to a $\mathrm{H}^{+}$(or $\left.\mathrm{He}^{+}\right)$ion, which becomes neutral. CT reactions between metal species are neglected because they are of minor importance compared to $\mathrm{H}$ and $\mathrm{He} \mathrm{CT}$ processes. Contrary to $\mathrm{CI}, \mathrm{RR}$, and DR processes, CT is generally regarded as a secondary effect leading to only moderate changes in the ionization balance. The four different CT reaction types are

$\mathrm{X}+\mathrm{H}^{+} \rightarrow \mathrm{X}^{+}+\mathrm{H}$

for hydrogen $\mathrm{CT}$ ionization of concerned species $\mathrm{X}=\mathrm{D}, \mathrm{He}, \mathrm{C}$, $\mathrm{N}, \mathrm{O}, \mathrm{Mg}, \mathrm{Mg}^{+}, \mathrm{Si}, \mathrm{Si}^{+}, \mathrm{Fe}, \mathrm{Fe}^{+}, \mathrm{H}_{2}, \mathrm{O}_{2}, \mathrm{OH}$, and $\mathrm{H}_{2} \mathrm{O}$;

$\mathrm{X}+\mathrm{He}^{+} \rightarrow \mathrm{X}^{+}+\mathrm{He}$,

for helium CT ionization of concerned species $\mathrm{X}=\mathrm{D}, \mathrm{Si}, \mathrm{H}_{2}, \mathrm{O}_{2}$, $\mathrm{OH}$, and $\mathrm{H}_{2} \mathrm{O}$;

$\mathrm{X}^{+}+\mathrm{H} \rightarrow \mathrm{X}+\mathrm{H}^{+}$,

for hydrogen CT recombination of concerned species $\mathrm{X}=\mathrm{D}, \mathrm{He}$, $\mathrm{C}, \mathrm{C}^{+}, \mathrm{C}^{2+}, \mathrm{C}^{3+}, \mathrm{N}, \mathrm{N}^{+}, \mathrm{N}^{2+}, \mathrm{N}^{3+}, \mathrm{O}, \mathrm{O}^{+}, \mathrm{O}^{2+}, \mathrm{O}^{3+}, \mathrm{Ne}^{+}, \mathrm{Ne}^{2+}$, $\mathrm{Ne}^{3+}, \mathrm{Mg}^{+}, \mathrm{Mg}^{2+}, \mathrm{Mg}^{3+}, \mathrm{Si}^{+}, \mathrm{Si}^{2+}, \mathrm{Si}^{3+}, \mathrm{Fe}^{+}, \mathrm{Fe}^{2+}, \mathrm{Fe}^{3+}, \mathrm{H}_{2}$, and $\mathrm{CO}$; and

$\mathrm{X}^{+}+\mathrm{He} \rightarrow \mathrm{X}+\mathrm{He}^{+}$,

for helium CT recombination of concerned species $\mathrm{X}=\mathrm{D}, \mathrm{C}^{2+}$, $\mathrm{C}^{3+}, \mathrm{N}^{+}, \mathrm{N}^{2+}, \mathrm{N}^{3+}, \mathrm{O}^{+}, \mathrm{O}^{2+}, \mathrm{O}^{3+}, \mathrm{Ne}^{+}, \mathrm{Ne}^{2+}, \mathrm{Ne}^{3+}, \mathrm{Mg}^{2+}, \mathrm{Mg}^{3+}$, $\mathrm{Si}^{2+}, \mathrm{Si}^{3+}, \mathrm{Fe}^{2+}$, and $\mathrm{Fe}^{3+}$. CT rate coefficients come from different sources as specified in Table A.1. In particular, rates for the reactions involving lower ionized metal species are taken from the Stancil et al. (2015) web database ${ }^{2}$, which catalogs data from previous works (e.g., Kingdon \& Ferland 1996).

Atomic data and fitting functions for metal species in the $\mathrm{NC}^{2} \mathrm{M}$ are probably very similar, and in parts identical, to those used in other investigations. For example, the ionization model of Oppenheimer \& Schaye (2013a) and the code Cloudy version 10 both apply the same Voronov (1997) fits for the CI rate coefficients and the same Badnell (2006) fits for RR and DR rate coefficients. Moreover, $\mathrm{CT}$ rates for many reactions in the $\mathrm{NC}^{2} \mathrm{M}$ with non-molecular species are likely identical to those in Oppenheimer \& Schaye (2013a) since coefficients originate more or less from the same literature sources. Based on data common ground, the equilibrium ionization structure of metals in the high-temperature regime is therefore expected to be very similar. The ionization structure in the low-temperature regime, on the other hand, is more difficult since many processes involving molecules play a role, and calculations depend much more on model details.

\subsection{2. $\mathrm{H}_{2}$ chemistry}

Molecular hydrogen is the most abundant molecule in the ISM, and it controls much of the chemistry running at a lower gas temperature. Large $\mathrm{H}_{2}$ fractions are observed in dense and dusty regions where its formation is dictated by reactions on the

\footnotetext{
2 https://wWW. physast. uga.edu/ugacxdb/
} 
surface of interstellar dust grains, which act as catalysts. Such dust-surface-catalytic reactions are considered in more detail in Sect. 2.3. In the absence of dust, generally smaller $\mathrm{H}_{2}$ fractions are produced by pure gas-phase reactions.

Since the two-body association process $2 \mathrm{H} \rightarrow \mathrm{H}_{2}$ is excluded, there remain two principle $\mathrm{H}_{2}$ formation channels in the gas phase. The first pathway is initiated by the $\mathrm{H}^{-}$ion, which is produced by the radiative association reaction

$\mathrm{H}+\mathrm{e}^{-} \rightarrow \mathrm{H}^{-}(+h v)$.

$\mathrm{H}_{2}$ is then formed by associative detachment with $\mathrm{H}$ according to

$\mathrm{H}^{-}+\mathrm{H} \rightarrow \mathrm{H}_{2}+\mathrm{e}^{-}$.

This two-step $\mathrm{H}_{2}$ formation sequence is probably the most important gas-phase mechanism in warm interstellar gas with sufficient fractional ionization. However, the sequence is a relatively slow process hampered by the usually low abundance of $\mathrm{H}^{-}$and in general cannot compete with $\mathrm{H}_{2}$ grain catalysis. In addition to the above fundamental reactions, a couple of further reactions are taken into account involving also the ions $\mathrm{H}_{2}^{+}$ and $\mathrm{H}_{3}^{+}$. Some of the reactions contribute to $\mathrm{H}_{2}$ formation like charge transfer from $\mathrm{H}$ to $\mathrm{H}_{2}^{+}$(reaction 303) or the dissociative detachment of $\mathrm{H}_{3}^{+}$with free electrons and $\mathrm{H}$ (reactions 323 , $325)$. Other reactions are counterproductive, like the mutual neutralization of $\mathrm{H}^{-}$with $\mathrm{H}^{+}$(reaction 309), which competes with associative detachment above, or charge transfer from $\mathrm{H}_{2}$ to $\mathrm{H}^{+}$ (reaction 304).

At sufficiently high gas densities ( such as $n \gtrsim 10^{16} \mathrm{~m}^{-3}$ ), a second pathway for $\mathrm{H}_{2}$ formation is by ter-molecular reactions. Here, the following three important reactions are taken into account:

$2 \mathrm{H}+\mathrm{X} \rightarrow \mathrm{H}_{2}+\mathrm{X}$,

where $\mathrm{X}=\mathrm{H}, \mathrm{He}, \mathrm{H}_{2}$.

The $\mathrm{H}_{2}$ subnetwork in the $\mathrm{NC}^{2} \mathrm{M}$ has been compiled from the chemical networks in Glover \& Abel (2008) and GFM10. Reaction rates are adopted from those works except for some reactions that apply more recent rates: reaction 301 uses the rate from Bruhns et al. (2010), reaction 305 uses the rate from Trevisan \& Tennyson (2002), and reaction 316 uses the UMIST RATE12 value (McElroy et al. 2013). RSO14 employs a similar set of reactions to treat gas-phase $\mathrm{H}_{2}$ formation and destruction processes, except that reaction 328 of the $\mathrm{NC}^{2} \mathrm{M}$, describing detachment of $\mathrm{H}^{-}$with $\mathrm{He}$, is missing in RSO14.

\subsubsection{HD chemistry}

The hydrogen reaction family is supplemented by a mininetwork for HD formation as suggested by Glover \& Jappsen (2007). Once molecular hydrogen is present it has been argued that the proton interchange reactions

$\mathrm{H}_{2}+\mathrm{D}^{+} \rightleftarrows \mathrm{HD}+\mathrm{H}^{+}$

are the most significant reactions in regulating the amount of HD, if the gas is sufficiently ionized. The importance of this reaction pair stems from the fact that below a few hundred Kelvin chemical fractionation can occur meaning a substantial enhancement of the $\mathrm{HD}: \mathrm{H}_{2}$ abundance ratio relative to the prevalent $\mathrm{D}: \mathrm{H}$ ratio. At higher temperatures, the analog neutral-neutral reactions

$\mathrm{H}_{2}+\mathrm{D} \rightleftarrows \mathrm{HD}+\mathrm{H}$ may also influence the HD abundance. These two pairs of principle reactions together with ionization, recombination, and CT reactions for D build the subnetwork to handle deuterium chemistry in the $\mathrm{NC}^{2} \mathrm{M}$. This network obviously neglects many D-bearing analogs to $\mathrm{H}$-bearing species like molecular deuterium, $\mathrm{D}_{2}$, deuterated hydronium, $\mathrm{D}_{3} \mathrm{O}^{+}$, or the ions $\mathrm{D}^{-}$and $\mathrm{HD}^{+}$. Reactions of $\mathrm{D}^{-}$and $\mathrm{HD}^{+}$with hydrogen, in particular, would provide an additional, albeit minor, source of HD formation analog to the $\mathrm{H}^{-} / \mathrm{H}_{2}^{+}$channel of $\mathrm{H}_{2}$ formation. Such a more detailed treatment of deuterium chemistry, which is not an intended goal here, can be found in Rodgers \& Millar (1996), for example.

\subsubsection{Carbon and oxygen chemistry}

The gas-phase carbon and oxygen chemistry in the $\mathrm{NC}^{2} \mathrm{M}$ is based on the RSO14 compilation of reactions. The RSO14 compilation, in turn, is built upon the network presented in GFM10. RSO14 added three reactions to the GFM10 model, which they claimed to be of significance for the $\mathrm{CO}$ formation process discovered by comparison with the Cloudy software. The $\mathrm{NC}^{2} \mathrm{M}$ accounts for these reactions $(483,510$, and 511 in Table A.1) using the rates given in RSO14. Other reaction rates are adopted from the GFM10 compilation with some exceptions: reactions 400, 404, 419, 437, 449, 454, 477, 479, 480-482, 488, 489, 491, 492, and 515 use updated rates from the UMIST RATE12 compilation. Reaction 493 applies the rate given in Martinez et al. (2008). The dissociative electron recombination reactions 498500 with hydronium make use of novel rates from Novotny et al. (2010). Finally, reactions 504 and 505 use more recent rates from Klippenstein \& Georgievskii (2010).

The applied network has been designed to accurately follow the abundances of the major molecular coolants $\mathrm{CO}$ and $\mathrm{H}_{2} \mathrm{O}$. It includes up to four-atom molecular species involved in their formation and destruction processes. Nevertheless, the network represents a considerable simplification with respect to comprehensive astrochemistry databases like UMIST. However, GFM10 demonstrated that this network is able to adequately model the abundances of $\mathrm{C}$-bearing and O-bearing species when compared to a more extensive network derived from the UMIST RATE99 database, if equal reaction rates are assumed. Moreover, Glover \& Clark (2012) have examined several approximate networks with different levels of detail for modeling CO chemistry among which the GFM10 network was the most detailed.

The complexity of chemical processes in diffuse and dense interstellar gas has been discussed in Herbst \& Klemperer (1973) and Dalgarno \& Black (1976) including C-,N-,O-,S-,Cl-, Si-bearing molecules. The network in the $\mathrm{NC}^{2} \mathrm{M}$ circumscribes the dominant chemical cycles for $\mathrm{C}$-bearing and O-bearing molecules, ignoring chemical schemes of the other elements. For the formation of $\mathrm{CO}$ and $\mathrm{H}_{2} \mathrm{O}$ in $\mathrm{H}_{2}$-dominated regions, intermediate molecules and its ionized versions such as $\mathrm{OH}, \mathrm{OH}^{+}$, the hydrocarbons $\mathrm{CH}_{x}, \mathrm{CH}_{x}^{+}$and the ions $\mathrm{HCO}^{+}$and $\mathrm{HOC}^{+}$ play a role. The major route to form $\mathrm{H}_{2} \mathrm{O}$ is initiated by the reactions

$\mathrm{O}^{+}+\mathrm{H}_{2} \rightarrow \mathrm{OH}^{+}+\mathrm{H}$

$\mathrm{O}+\mathrm{H}_{3}^{+} \rightarrow \mathrm{OH}^{+}+\mathrm{H}_{2}$

forming the hydroxyl ion. This is followed by the sequence

$\mathrm{OH}^{+}+\mathrm{H}_{2} \rightarrow \mathrm{H}_{2} \mathrm{O}^{+}+\mathrm{H}$

$\mathrm{H}_{2} \mathrm{O}^{+}+\mathrm{H}_{2} \rightarrow \mathrm{H}_{3} \mathrm{O}^{+}+\mathrm{H}$ 
and subsequent dissociative recombinations

$$
\begin{aligned}
\mathrm{OH}^{+}+\mathrm{e}^{-} & \rightarrow \mathrm{O}+\mathrm{H} \\
\mathrm{H}_{2} \mathrm{O}^{+}+\mathrm{e}^{-} & \rightarrow \mathrm{OH}+\mathrm{H} \\
& \rightarrow \mathrm{O}+\mathrm{H}_{2} \\
\mathrm{H}_{3} \mathrm{O}^{+}+\mathrm{e}^{-} & \rightarrow \mathrm{O}+2 \mathrm{H} \\
& \rightarrow \mathrm{H} \mathrm{H}_{2} \mathrm{OH}+\mathrm{H}_{2} \\
& \rightarrow \mathrm{OH}+2 \mathrm{H} \\
& \rightarrow \mathrm{O}+\mathrm{H}_{2}+\mathrm{H} .
\end{aligned}
$$

Where the level of ionization is low, the following neutralneutral reactions contribute to $\mathrm{H}_{2} \mathrm{O}$ formation:

$$
\begin{aligned}
& \mathrm{OH}+\mathrm{H} \rightarrow \mathrm{H}_{2} \mathrm{O} \\
& \mathrm{OH}+\mathrm{H}_{2} \rightarrow \mathrm{H}_{2} \mathrm{O}+\mathrm{H} \\
& 2 \mathrm{OH} \rightarrow \mathrm{H}_{2} \mathrm{O}+\mathrm{O} .
\end{aligned}
$$

Important routes to form $\mathrm{CO}$ are by the reactions

$$
\begin{aligned}
\mathrm{C}^{+}+\mathrm{OH} & \rightarrow \mathrm{CO}+\mathrm{H}^{+} \\
& \rightarrow \mathrm{CO}^{+}+\mathrm{H}
\end{aligned}
$$

followed by

$$
\begin{aligned}
& \mathrm{CO}^{+}+\mathrm{H}_{2} \rightarrow \mathrm{HCO}^{+}+\mathrm{H} \\
& \mathrm{HCO}^{+}+\mathrm{e}^{-} \rightarrow \mathrm{CO}+\mathrm{H},
\end{aligned}
$$

and by the reactions

$$
\begin{aligned}
& \mathrm{O}+\mathrm{CH}^{+} \rightarrow \mathrm{CO}+\mathrm{H}^{+} \\
& \mathrm{O}+\mathrm{C}_{2}^{+} \rightarrow \mathrm{CO}+\mathrm{C}^{+},
\end{aligned}
$$

where the formation and destruction of hydrocarbon radicals up to $\mathrm{CH}_{3}^{+}$is governed by numerous reactions not discussed here, but fully included in the $\mathrm{NC}^{2} \mathrm{M}$. Further minor sources of $\mathrm{CO}$ are the dissociative recombination

$\mathrm{HOC}^{+}+\mathrm{e}^{-} \rightarrow \mathrm{CO}+\mathrm{H}$

the ion-neutral reactions

$\mathrm{HCO}^{+}+\mathrm{C} \rightarrow \mathrm{CO}+\mathrm{CH}^{+}$

$\mathrm{HCO}^{+}+\mathrm{H}_{2} \mathrm{O} \rightarrow \mathrm{CO}+\mathrm{H}_{3} \mathrm{O}^{+}$,

and the charge transfer reaction

$\mathrm{CO}^{+}+\mathrm{H} \rightarrow \mathrm{CO}+\mathrm{H}^{+}$.

In very dense regions, three-body molecular associations with neutral helium acting as catalyst may become important. The $\mathrm{NC}^{2} \mathrm{M}$ includes a couple of reactions of this type that are numbered 519-525 in Table A.1.

\subsection{Cosmic ray reactions}

Cosmic rays (CR) are such highly energetic particles that they can deeply penetrate dense gas regions that are opaque to ionizing ultraviolet (UV) radiation otherwise. CR-induced ionizations of $\mathrm{H}, \mathrm{H}_{2}$, and $\mathrm{He}$ maintain a sufficient level of free electrons and so act as a driver for ion-molecule chemistry at low gas temperatures. The $\mathrm{NC}^{2} \mathrm{M}$ takes into account two distinct types of cosmic ray reactions: primary and secondary. Primary reactions (labeled "cr"-reactions) denote the direct impact of a cosmic ray particle resulting in the ionization of a specie $\mathrm{X}$ or the dissociation of $\mathrm{H}_{2}$,

$\mathrm{X} \stackrel{\text { cr }}{\rightarrow} \mathrm{X}^{+}+\mathrm{e}^{-}$,

$\mathrm{H}_{2} \stackrel{\text { cr }}{\rightarrow} 2 \mathrm{H}$.

Secondary reactions (labeled " $\gamma_{\mathrm{cr}}$ "-reactions) denote photoreactions due to ultraviolet photons generated internally by the interaction of energetic electrons ejected into primary cosmic ray ionizations with the gas. Secondary reactions are the photoionization of species $\mathrm{X}$ and the photodissociation of molecules XY,

$\mathrm{X} \stackrel{\gamma_{\mathrm{cr}}}{\rightarrow} \mathrm{X}^{+}+\mathrm{e}^{-}$,

$\mathrm{XY} \stackrel{\gamma_{\text {cr }}}{\rightarrow} \mathrm{X}+\mathrm{Y}$.

A summary of all CR-induced reactions included in the $\mathrm{NC}^{2} \mathrm{M}$ is given in Table A.2. The subset of cosmic ray reactions is nearly equivalent to RSO14 but the rate coefficients differ for some reactions because of the dissimilar handling of secondary effects described below.

The primary cosmic ray ionization rate, $\zeta$, is dominated by the energy spectrum of cosmic ray particles in the lower energy regime $(\lesssim 100 \mathrm{MeV})$. Unfortunately, this part of the energy spectrum is observationally not well determined. For the cosmic ray ionization rate of hydrogen, the value of $\zeta_{\mathrm{H}}=2.5 \times 10^{-17} \mathrm{~s}^{-1}$ from Williams et al. 1998 is adopted in order to allow comparison with other work. However, more recent observations of $\mathrm{H}_{3}^{+}$in the Galactic diffuse ISM indicate that the rate may be higher by one order of magnitude (Indriolo \& McCall 2012). The ionization rates for other species are assumed to be linearly correlated,

$\zeta=\zeta^{\mathrm{rel}} \zeta_{\mathrm{H}}$.

The numbers $\zeta^{\text {rel }}$ are extracted from the UMIST RATE12 database insofar available. For all other "cr"-reactions, the factors are estimated on the basis of the theoretical considerations in Silk (1970) and Lotz (1967). Namely,

$\zeta^{\mathrm{rel}}=\bar{\xi} \frac{\chi_{\mathrm{H}}}{\chi}$,

where $\chi_{\mathrm{H}}$ and $\chi$ are the ionization energies of hydrogen and the species in mind, respectively, and $\bar{\xi}$ denotes the outer shell effective electron number of that species given by

$\bar{\xi}=\bar{\chi} \sum_{j=1}^{2} \frac{\xi_{j}}{\chi_{j}}, \quad \ln \bar{\chi}=\frac{\sum_{j=1}^{2} \xi_{j} / \chi_{j} \ln \chi_{j}}{\sum_{j=1}^{2} \xi_{j} / \chi_{j}}$.

Here, $\xi_{j}$ and $\chi_{j}$ are the number of electrons and ionization potentials of the outermost $(j=1)$ and next inner $(j=2)$ subshell of the species. The various values can be found in Table 1 in Lotz (1967).

The ejected electron in a primary ionization has an average energy of about $30 \mathrm{eV}$ for cosmic ray particles in the energy range $10 \ldots 100 \mathrm{MeV}$ (Cravens \& Dalgarno 1978). Such energetic electrons interacting with the gas can give rise to secondary effects, which are of potential significance especially in low-temperature, high-density environments: further ionization, collisional excitation of atoms and molecules, dissociation of molecules, and Coloumb scattering in partly ionized gas. Dalgarno et al. (1999) have made detailed investigations of the energy degradation process of energetic electrons in a partly ionized $\mathrm{H}_{2}-\mathrm{H}-\mathrm{He}$ gas mixture ${ }^{3}$ for incident electron energies up to $1 \mathrm{keV}$. To account for such secondary effects in the $\mathrm{NC}^{2} \mathrm{M}$, their fitting formulae for a $30 \mathrm{eV}$ electron have been elaborated. This

3 With a fixed helium fraction of $n_{\mathrm{He}}=0.1\left(2 n_{\mathrm{H}_{2}}+n_{\mathrm{H}}\right)$. 
way the number of secondary ionizations of $\mathrm{H}$ and $\mathrm{H}_{2}$, the yields of $\mathrm{H}(2 p)$ excitations (Ly $\alpha$ photons), and the yields of electronic excitations of $\mathrm{H}_{2}$ (Lyman-Werner band photons) are computed as functions of the number density ratio $n_{\mathrm{H}_{2}} / n_{\mathrm{H}}$ and ionization degree. The net (primary plus secondary) cosmic ray ionization rate for atomic and molecular hydrogen is therefore represented by

$\zeta_{\mathrm{H}}^{\mathrm{tot}}=\left(1+N_{\mathrm{H}^{+}}^{\mathrm{sec}}\right) \zeta_{\mathrm{H}}$,

$\zeta_{\mathrm{H}_{2}}^{\mathrm{tot}}=\left(1+N_{\mathrm{H}_{2}^{+}}^{\mathrm{sec}}\right) \zeta_{\mathrm{H}_{2}}$,

where the numbers of secondary ionizations are

$$
\begin{aligned}
& N_{\mathrm{H}^{+}}^{\mathrm{sec}} \approx 0.5 x_{\mathrm{H}}\left[1+221\left(\frac{n_{\mathrm{e}}}{0.2 n_{\mathrm{H}_{2}}+1.1 n_{\mathrm{H}}}\right)^{1.06}\right]^{-1}, \\
& N_{\mathrm{H}_{2}^{+}}^{\mathrm{sec}} \approx 0.76 x_{\mathrm{H}_{2}}\left[1+202\left(\frac{n_{\mathrm{e}}}{1.2 n_{\mathrm{H}_{2}}+0.1 n_{\mathrm{H}}}\right)^{1.15}\right]^{-1},
\end{aligned}
$$

with $x_{\mathrm{H}}=n_{\mathrm{H}} /\left(2 n_{\mathrm{H}_{2}}+n_{\mathrm{H}}\right)$ and $x_{\mathrm{H}_{2}}=n_{\mathrm{H}_{2}} /\left(2 n_{\mathrm{H}_{2}}+n_{\mathrm{H}}\right)$. Dalgarno et al. (1999) state that the expressions for $N_{\mathrm{H}^{+}}^{\mathrm{sec}}$ and $N_{\mathrm{H}_{2}^{+}}^{\mathrm{sec}}$ are accurate for ionization degrees up to $10 \%$. For a neutral $\mathrm{H}-$ He gas mixture $\left(x_{\mathrm{H}}=1, n_{\mathrm{e}}=0\right) N_{\mathrm{H}^{+}}^{\mathrm{sec}} \approx 0.5$, whereas for a neutral $\mathrm{H}_{2}-\mathrm{He}$ gas mixture $\left(x_{\mathrm{H}_{2}}=0.5, n_{\mathrm{e}}=0\right) N_{\mathrm{H}_{2}^{+}}^{\mathrm{sec}} \approx 0.38$. The number of secondary ionizations decreases with increasing ionization degree because a larger fraction of the ejected electron energy goes into gas heating via Coloumb scattering lowering the yields of ionizations and excitations. At a $10 \%$ ionization level the number of secondary ionizations is reduced by more than one order of magnitude compared to neutral gas. Secondary ionizations of $\mathrm{He}$ (and also metals) are ignored because their contribution to the ionization rate is small. In the case of the above neutral $\mathrm{H}-\mathrm{He}$ gas mixture, we find $N_{\mathrm{He}^{+}}^{\mathrm{sec}} \approx 5 \times 10^{-3}$, and $N_{\mathrm{He}^{++}}^{\mathrm{sec}}=0$ since the necessary ionization energy of $54.4 \mathrm{eV}$ is above the ejected electron energy of $30 \mathrm{eV}$. In RSO14 the hydrogen secondary ionization rate is computed from data tables in Furlanetto \& Stoever (2010) obtained by Monte-Carlo simulations of the energy degradation problem. However, these simulations were done for atomic gas and are therefore not suited to estimate secondary ionization rates in a $\mathrm{H}-\mathrm{H}_{2}$ gas mixture.

Gredel et al. (1987, 1989) have studied CR-induced photoionization and photodissociation for a variety of interstellar molecules. The authors determined photoreaction rates on the basis of detailed calculations of the emission spectrum resulting from the impact excitation of $\mathrm{H}_{2}$ by a $30 \mathrm{eV}$ electron. Accordingly, the photoreaction rate $R_{\mathrm{X}}$ in units $\mathrm{s}^{-1}$ for a species $\mathrm{X}$ is given by

$R_{\mathrm{X}}=\frac{p_{\mathrm{X}}}{1-\omega} \zeta_{\mathrm{H}_{2}}^{\mathrm{tot}}$

where $p_{\mathrm{X}}$ is the efficiency for reactions with Lyman-Werner photons per total number of ionizations and $\omega$ is the grain albedo ( $\omega=0.5$ is used throughout). In computing $p_{\mathrm{X}}$ a pure neutral $\mathrm{H}_{2}$ gas with $N_{\mathrm{H}_{2}^{+}}^{\mathrm{sec}}=0.55$ was assumed, and yields of 0.3 and 0.12 for the dominating excitations to the $B^{1} \Sigma_{\mathrm{u}}^{+}$(including cascading contribution from higher excited states) and $C^{1} \Pi_{\mathrm{u}}$ electronic states of $\mathrm{H}_{2}$ were adopted. For the $\mathrm{NC}^{2} \mathrm{M}$, the Gredel et al. outcome has been recalibrated to the situation of a partially ionized $\mathrm{H}-\mathrm{H}_{2}-\mathrm{He}$ gas mixture making use of the energy degradation results in Dalgarno et al. (1999) a second time. If atomic hydrogen is present, excitations to the $\mathrm{H}(2 p)$ state followed by Ly $\alpha$ emission contribute to the generated UV flux. From the fitting formulae in Dalgarno et al. (1999), the yields for Lyman-Werner photons and Ly $\alpha$ photons are approximately given by

$Y_{\mathrm{LyW}} \approx 1.09 N_{\mathrm{H}_{2}^{+}}^{\mathrm{sec}}, \quad Y_{\mathrm{Ly} \alpha} \approx 1.8 N_{\mathrm{H}^{+}}^{\mathrm{sec}}$.

This suggest the following modified photoreaction rate:

$R_{\mathrm{X}}=\left(\frac{1.55}{0.42} Y_{\mathrm{Lyw}} p_{\mathrm{X}}+Y_{\mathrm{Ly} \alpha} q_{\mathrm{X}}\right)(1-\omega)^{-1} \bar{\zeta}$,

where $q_{\mathrm{X}}$ is the efficiency of a species $\mathrm{X}$ for reactions with Ly $\alpha$ photons. Values for various species can be found in Maloney et al. (1996). The averaged primary ionization rate, $\bar{\zeta}$, is given by

$\bar{\zeta}=\frac{n_{\mathrm{H}} \zeta_{\mathrm{H}}+n_{\mathrm{H}_{2}} \zeta_{\mathrm{H}_{2}}}{n_{\mathrm{H}}+n_{\mathrm{H}_{2}}}$.

We note that $R_{\mathrm{X}}$ now depends on the ionization degree because the yields $Y_{\mathrm{LyW}}$ and $Y_{\mathrm{Ly} \alpha}$ do so. For a neutral $\mathrm{H}_{2}-\mathrm{He}$ gas mixture $\left(x_{\mathrm{H}_{2}}=0.5, n_{\mathrm{He}}=0.2 n_{\mathrm{H}_{2}}, n_{\mathrm{e}}=0\right)$, the rate reduces to

$R_{X}=\frac{p_{\mathrm{X}}}{1-\omega} 1.53 \zeta_{\mathrm{H}_{2}}$

which is close to the Gredel et al. (1989) result where $\zeta_{\mathrm{H}_{2}}^{\text {tot }}=$ $1.55 \zeta_{\mathrm{H}_{2}}$. The efficiencies $p_{\mathrm{X}}$ and $q_{\mathrm{X}}$ for all CR-induced photoreactions in the $\mathrm{NC}^{2} \mathrm{M}$ are summarized in Table A.2.

As discussed in Gredel et al. (1987), the efficiency $p_{\mathrm{CO}}$ of CO for reactions with Lyman-Werner photons must be treated more carefully since efficient destruction of $\mathrm{CO}$ occurs only if close coincidences between $\mathrm{H}_{2}$ emission lines and $\mathrm{CO}$ absorption lines exist. The efficiency $p_{\mathrm{CO}}$ is tabulated in Gredel et al. (1987) as a function of temperature, $\mathrm{CO}$ abundance $x_{\mathrm{CO}}$, and grain albedo. For a grain albedo of 0.5 a crude interpolation is $p_{\mathrm{CO}} \approx 1.35\left(T / x_{\mathrm{CO}}\right)^{0.5}$. The CR-induced $\mathrm{CO}$ photodissociation rate is then approximated by

$R_{\mathrm{CO}}=\frac{1.55}{0.42} Y_{\mathrm{LyW}} p_{\mathrm{CO}} \bar{\zeta}$

This approaches the original Gredel et al. (1987) result, $R_{\mathrm{CO}}=$ $p_{\mathrm{CO}} 1.55 \zeta_{\mathrm{H}_{2}}$, if electron degradation is assumed to take place in a neutral $\mathrm{H}_{2}$ gas where $Y_{\mathrm{LyW}} \approx 0.41$ according to Dalgarno et al. (1999).

\subsection{Dust-catalytic reactions}

To understand the high fraction of molecular hydrogen observed in various interstellar environments, dust grain chemistry seems indispensable. The $\mathrm{NC}^{2} \mathrm{M}$ adheres to the fundamental process of $\mathrm{H}_{2}$ formation on the surface of dust particles. Moreover, several relevant grain-surface ion recombination reactions are considered. A compilation of all dust-catalytic reactions is given in Table A.3. The efficacy of surface reactions depends on the total cross section of the dust mixture. A static dust model is predisposed, that is, the current $\mathrm{NC}^{2} \mathrm{M}$ implementation does not account for dust destruction (sputtering, sublimation) and formation (accretion, coagulation) phenomena. Therefore, the total dust cross section does not change with time. Also, the implementation of ion-dust recombination assumes that neutralized ions re-enter the gas phase after charge transfer and are not adsorbed onto the grain. Treatment of such element depletion processes is currently beyond the capabilities of the $\mathrm{NC}^{2} \mathrm{M}$.

The $\mathrm{NC}^{2} \mathrm{M}$ applies the dust model of Weingartner \& Draine (2001a), which reproduces the observed extinction curve and 
dust emission spectrum of the Milky Way by a mixture of graphite, PAHs, and silicate spherical grains with distinct size distributions $n_{\mathrm{d}}(a)$ ( $a$ : grain radius). According to Draine (2002) the total dust cross-sectional area per unit volume for this model is

$\left(n_{\mathrm{d}} \sigma_{\mathrm{d}}\right)_{\mathrm{tot}}=\int \mathrm{d} a \frac{\mathrm{d} n_{\mathrm{d}}}{\mathrm{d} a} \pi a^{2}=6.7 \times 10^{-25} \mathrm{~m}^{2} \cdot n_{\mathrm{H}_{\mathrm{nuc}}}$,

with $n_{\mathrm{H}_{\text {nuc }}}$ being the number density of $\mathrm{H}$ nuclei ${ }^{4}$. For a dustto-gas ratio other than the average value in the Milky Way, it is common to scale $\left(n_{\mathrm{d}} \sigma_{\mathrm{d}}\right)_{\text {tot }}$ linearly with the metallicity $Z$, hence, $\left(n_{\mathrm{d}} \sigma_{\mathrm{d}}\right)_{\mathrm{tot}}=6.7 \times 10^{-25} \mathrm{~m}^{2} \cdot n_{\mathrm{H}_{\mathrm{nuc}}}\left(Z / Z_{\odot}\right)$.

The rate of $\mathrm{H}_{2}$ formation according to reaction $700, \dot{n}_{\mathrm{H}_{2}}=$ $R_{\mathrm{H}_{2}}=k_{700} n_{\mathrm{H}}^{2}$, can be expressed as (see, e.g., Cazaux \& Tielens 2002)

$R_{\mathrm{H}_{2}}=\frac{1}{2} n_{\mathrm{H}} v_{\mathrm{th}, \mathrm{H}}\left(n_{\mathrm{d}} \sigma_{\mathrm{d}}\right)_{\mathrm{tot}} S_{\mathrm{H}} \epsilon_{\mathrm{H}_{2}}$,

where

$v_{\mathrm{th}, \mathrm{H}}=\sqrt{\frac{8 k T}{\pi m_{\mathrm{H}}}}$

is the mean thermal speed of the $\mathrm{H}$ gas component and $S_{\mathrm{H}}\left(T, T_{\mathrm{d}}\right)$ is the sticking probability for adsorbing $\mathrm{H}$ atoms, which generally depends on both the gas temperature $T$ and the dust temperature $T_{\mathrm{d}}$. The sticking coefficient is taken from Hollenbach \& McKee (1979)

$S_{\mathrm{H}}\left(T, T_{\mathrm{d}}\right)=\left[1+0.04 \sqrt{\frac{T+T_{\mathrm{d}}}{\mathrm{K}}}+0.002 \frac{T}{\mathrm{~K}}+8 \times 10^{-6}\left(\frac{T}{\mathrm{~K}}\right)^{2}\right]^{-1}$.

The function $\epsilon_{\mathrm{H}_{2}}$ describes the surface recombination efficiency, which is the fraction of accreted $\mathrm{H}$ atoms that leave the surface as $\mathrm{H}_{2}$. The $\mathrm{NC}^{2} \mathrm{M}$ adopts the general expression in Cazaux \& Tielens (2002), Eq. (15). This expression follows from the rate equation model of Cazaux \& Tielens (2002, 2004) describing grain surface abundances in physisorbed and chemisorbed sites subject to different surface processes (desorption, migration: thermal diffusion and quantum mechanical tunneling, reaction). Using values for the surface characteristics parameters between those for silicate and carbonaceous grain materials as cataloged in Cazaux \& Spaans (2004), one finds

$$
\begin{aligned}
\epsilon_{\mathrm{H}_{2}}= & {\left[1+7 \times 10^{-14} \mathrm{~s} F \exp \left(\frac{440 \mathrm{~K}}{T_{\mathrm{d}}}\right)+19 \exp \left(-\frac{225 \mathrm{~K}}{T_{\mathrm{d}}}\right)\right]^{-1} } \\
& \times\left[1+\frac{5.7 \times 10^{14} \mathrm{~s}^{-1}}{F} \exp \left(-\frac{45000 \mathrm{~K}}{T_{\mathrm{d}}}\right)\right]^{-1}
\end{aligned}
$$

where $F$ is the incoming $\mathrm{H}$ atom flux expressed in monolayers per second. This flux is approximated by

$F=\frac{1}{4} v_{\mathrm{th}, \mathrm{H}} n_{\mathrm{H}} 4 \pi a^{2} S_{\mathrm{H}} \frac{1}{N_{\mathrm{ass}}}=\frac{1}{4} v_{\mathrm{th}, \mathrm{H}} n_{\mathrm{H}} S_{\mathrm{H}} W^{2}$,

where $v_{\mathrm{th}, \mathrm{H}} n_{\mathrm{H}} \pi a^{2} S_{\mathrm{H}}$ is the number of $\mathrm{H}$ atoms per second landing on a spherical grain with radius $a$ and $N_{\text {ass }}=4 \pi a^{2} / W^{2}$ denotes the number of adsorption sites on the surface. A typical value for the horizontal barrier width between adjacent

\footnotetext{
4 This is a factor 6.7 larger than the total cross section used in RSO14, which was based on the dust UV absorption cross section per $\mathrm{H}$ nucleus mentioned in Krumholz et al. (2011).
}

adsorption sites is $W \approx 2 \AA$ (Cazaux \& Tielens 2004). For an order of magnitude estimate in cold cores, for instance, adopting $n=10^{10} \mathrm{~m}^{-3}$ and $T=T_{\mathrm{d}}=10 \mathrm{~K}$ gives a value of $F \approx 5 \times 10^{-8}$ monolayers per second. We note that $R_{\mathrm{H}_{2}}$ becomes negligible for higher gas temperatures since $v_{\mathrm{th}, \mathrm{H}} S_{\mathrm{H}} \propto T^{-1.5}$ for $T \gg 1000 \mathrm{~K}$.

A second relevant grain-surface process that codetermines the ionization balance in the low-temperature regime is the neutralization of ions. This process competes with radiative recombination for gas temperatures below a few thousand Kelvin and counteracts cosmic ray ionization in cold, UV-shielded regions. The $\mathrm{NC}^{2} \mathrm{M}$ takes into account ion recombination of the elements $\mathrm{X}=\mathrm{H}^{+}, \mathrm{He}^{+}, \mathrm{C}^{+}, \mathrm{Mg}^{+}, \mathrm{Si}^{+}$, and $\mathrm{Fe}^{+}$. The rate coefficients, $k_{\mathrm{X}}$, for reactions $701-706$ use the fitting formula from Weingartner \& Draine (2001b), Eq. (8),

$$
\dot{n}_{\mathrm{X}}=-k_{\mathrm{X}} n_{\mathrm{X}} \approx-\frac{10^{-20} C_{0} n_{\mathrm{H}_{n u c}} \mathrm{~m}^{3} \mathrm{~s}^{-1}}{1+C_{1} \tilde{\Psi}^{C_{2}}\left(1+C_{3}\left(\frac{T}{\mathrm{~K}}\right)^{C_{4}} \tilde{\Psi}^{-C_{5}-C_{6} \ln (T / \mathrm{K})}\right)} n_{\mathrm{X}},
$$

with the coefficients $C_{0}-C_{6}$ given in Table 2 of Weingartner \& Draine (2001b). This formula has been derived for the Milky Way dust model of Weingartner \& Draine (2001a), already used for $R_{\mathrm{H}_{2}}$, and accounts for grain charging expressed by the (dimensionless) charging parameter (see Weingartner \& Draine 2001c)

$\tilde{\Psi}=G \frac{10^{6} \mathrm{~m}^{-3}}{n_{\mathrm{e}}} \sqrt{\frac{T}{\mathrm{~K}}}$

The quantity $G$ is a measure of the radiation intensity (in units of the Habing radiation field energy density) responsible for photoelectric grain charging. Equation (22) is multiplied by an additional factor $Z / Z_{\odot}$ to account for different dust-to-gas ratios as has been done for $R_{\mathrm{H}_{2}}$. As stressed by Weingartner \& Draine (2001b), the expression for $k_{\mathrm{X}}$ likely becomes inaccurate when $\tilde{\Psi} \lesssim 10^{2}$. It is interesting to note that the limit $G \rightarrow 0$ $(\tilde{\Psi} \rightarrow 0$ ) would correspond to a completely radiation-free environment where grain charging is driven solely by electron collisional charging (ion collisional charging was neglected in Weingartner \& Draine 2001b) resulting in over-balanced negatively charged grains, and $k_{\mathrm{X}}$ becomes a maximum.

\section{Thermal processes}

In time-dependent, gas-dynamical simulations thermal processes enter the energy equation as a net volumetric cooling rate $\Lambda_{\text {net }}$ changing the energy density $\epsilon$,

$\frac{\mathrm{d} \epsilon}{\mathrm{d} t}=-\Lambda_{\text {net }}$,

where $\Lambda_{\text {net }}=\Lambda-\Gamma$ is the difference between total cooling $\left(\Lambda=\sum \Lambda_{i} \geq 0\right)$ and total heating $\left(\Gamma=\sum \Gamma_{i} \geq 0\right)$ rates. The individual cooling and heating contributions, $\Lambda_{i}$ and $\Gamma_{i}$, are described in more detail below. The heating processes include chemical heating associated with $\mathrm{H}_{2}$ formation reactions and cosmic ray heating. Among the cooling processes are chemical cooling due to ionization, recombination, and $\mathrm{H}_{2}$ dissociation, atomic line cooling of all elements, and molecular line cooling of $\mathrm{H}_{2}, \mathrm{CO}, \mathrm{H}_{2} \mathrm{O}$, and $\mathrm{OH}$. 


\subsection{Chemical heating and cooling}

The $\mathrm{NC}^{2} \mathrm{M}$ takes into account chemical heating of the gas through the exothermic reactions of $\mathrm{H}_{2}$ formation both in the gas phase and dust-assisted. A substantial part of released energy in these reactions goes into the excitation of rotovibrational states of the newly formed hydrogen molecule, which is then partly transformed into gas heating via collisional de-excitation. The energy fraction converted to heat by collisional de-excitation (rather than radiated away) depends on the gas density and is approximated in Hollenbach \& McKee (1979) by

$f=\left[1+\frac{10^{12}(T / \mathrm{K})^{-0.5} \mathrm{~m}^{-3}}{1.6 n_{\mathrm{H}} \exp \left[-\left(\frac{400 \mathrm{~K}}{T}\right)^{2}\right]+1.4 n_{\mathrm{H}_{2}} \exp \left(-\frac{12000 \mathrm{~K}}{T+1200 \mathrm{~K}}\right)}\right]^{-1}$.

Following Omukai (2000) and Hollenbach \& McKee (1979) the gas heating rates associated with $\mathrm{H}_{2}$ formation by the different processes, namely, $\mathrm{H}^{-} / \mathrm{H}_{2}^{+}$(reactions 301,303 ), three-body reactions $313,314,317$, and grain-catalytic reaction 700 are given by

$\Gamma_{301}=(3.53 \mathrm{eV}) f k_{301} n_{\mathrm{H}^{-}} n_{\mathrm{H}}$

$\Gamma_{303}=(1.83 \mathrm{eV}) f k_{303} n_{\mathrm{H}_{2}^{+}} n_{\mathrm{H}}$

$\Gamma_{313}=(4.48 \mathrm{eV}) f k_{313} n_{\mathrm{H}}^{3}$

$\Gamma_{314}=(4.48 \mathrm{eV}) f k_{314} n_{\mathrm{H}^{2}}^{2} n_{\mathrm{H}_{2}}$

$\Gamma_{317}=(4.48 \mathrm{eV}) f k_{317} n_{\mathrm{H}}^{2} n_{\mathrm{He}}$

$\Gamma_{700}=(0.2 \mathrm{eV}+4.2 \mathrm{eV} f) k_{700} n_{\mathrm{H}}^{2}$.

Reaction 301, 303 liberates $3.53 \mathrm{eV}(1.83 \mathrm{eV})$ of energy, and it is assumed that all go into rotovibrational excitation. The threebody reactions release the $\mathrm{H}_{2}$ binding energy of $4.48 \mathrm{eV}$, which is assumed to be stored initially in excitation as well. In the heating rate due to $\mathrm{H}_{2}$ formation on dust grains, $\Gamma_{700}$, it is assumed that $4.2 \mathrm{eV}$ of liberated binding energy goes into excitation, $0.2 \mathrm{eV}$ is thermalized kinetic energy of the desorbing molecule, and the remaining amount of energy is absorbed by the grain.

Collisional ionization of $\mathrm{H}, \mathrm{He}$, and $\mathrm{He}^{+}$(reactions $1,3,5$ ), radiative recombination of $\mathrm{H}^{+}$and $\mathrm{He}^{++}$(reactions 2, 6), radiative and di-electronic recombination of $\mathrm{He}^{+}$(reaction 4), and collisional dissociation of $\mathrm{H}_{2}$ (reactions $305,306,315$ ) are the most relevant chemical cooling processes. The associated cooling rates are compiled from different sources (Spitzer 1978; Janev et al. 1987; Cen 1992; Verner \& Ferland 1996; Omukai 2000):

$$
\begin{aligned}
\Lambda_{1}= & (13.60 \mathrm{eV}) k_{1} n_{\mathrm{H}} n_{\mathrm{e}} \\
\Lambda_{2}= & \alpha k_{\mathrm{B}} T k_{2} n_{\mathrm{H}^{+}} n_{\mathrm{e}} \\
\Lambda_{3}= & (24.59 \mathrm{eV}) k_{3} n_{\mathrm{He}} n_{\mathrm{e}}+5.01 \times 10^{-46} \frac{\mathrm{Jm}^{6}}{\mathrm{~s}} C(T) \\
& \times(T / \mathrm{K})^{-0.1687} \exp (-55338 \mathrm{~K} / T) n_{\mathrm{He}^{+}} n_{\mathrm{e}}^{2} \\
\Lambda_{4}= & \left(\alpha k_{\mathrm{B}} T k_{4, R R}+(40.8 \mathrm{eV}) k_{4, D R}\right) n_{\mathrm{He}^{+}} n_{\mathrm{e}} \\
\Lambda_{5}= & (54.42 \mathrm{eV}) k_{5} n_{\mathrm{He}^{+}} n_{\mathrm{e}} \\
\Lambda_{6}= & \alpha k_{\mathrm{B}} T k_{6} n_{\mathrm{He}^{++}} n_{\mathrm{e}} \\
\Lambda_{305}= & (4.48 \mathrm{eV}) k_{305} n_{\mathrm{H}_{2}} n_{\mathrm{e}} \\
\Lambda_{306}= & (4.48 \mathrm{eV}) k_{306} n_{\mathrm{H}_{2}} n_{\mathrm{H}} \\
\Lambda_{315}= & (4.48 \mathrm{eV}) k_{315} n_{\mathrm{H}_{2}}^{2} .
\end{aligned}
$$

In the recombination cooling coefficients $\Lambda_{2}, \Lambda_{4}$, and $\Lambda_{6}$, $\alpha k_{\mathrm{B}} T \approx 0.75 k_{\mathrm{B}} T$ is the mean kinetic energy of the captured electron ${ }^{5}$. The second term in $\Lambda_{3}$ accounts for collisional ionization cooling from the metastable $2^{3} S_{1}$ He state, which is proportional to $n_{\mathrm{He}^{+}} n_{\mathrm{e}}^{2}$ (Black 1981), and where $C(T)=(1+$ $\left.T / 10^{5} \mathrm{~K}\right)^{-1}$.

\subsection{Atomic line cooling}

\subsection{1. $\mathrm{H}$ and $\mathrm{He}$}

The $\mathrm{NC}^{2} \mathrm{M}$ takes into account radiation cooling from electron impact excitation of $\mathrm{H}, \mathrm{He}$, and $\mathrm{He}^{+}$. Rates for $\mathrm{H}$ and $\mathrm{He}^{+}$are taken from Cen (1992) based on the work of Black (1981). The cooling rate for $\mathrm{He}^{+}$only covers excitation to the $n=2$ atomic level, whereas the rate for $\mathrm{H}$ covers all $n$. The rate for neutral He distinguishes between excitation from the ground state $1^{1} S_{0}$, which is approximated by the function in Glover \& Jappsen (2007), Table 7, based on data from Bray et al. (2000), and excitation from the metastable $2^{3} S_{1}$ state to $n=2,3,4$ triplet states adopting the rate from Cen (1992). The rates can be written as

$$
\begin{aligned}
\Lambda_{\mathrm{H}}= & 7.5 \times 10^{-32} \frac{\mathrm{Jm}^{3}}{\mathrm{~s}} C(T) \exp \left(-\frac{118348 \mathrm{~K}}{T}\right) n_{\mathrm{H}} n_{\mathrm{e}} \\
\Lambda_{\mathrm{He}}= & 1.1 \times 10^{-32} \frac{\mathrm{Jm}^{3}}{\mathrm{~s}}\left(\frac{T}{\mathrm{~K}}\right)^{0.082} \exp \left(-\frac{230000 \mathrm{~K}}{T}\right) n_{\mathrm{He}} n_{\mathrm{e}} \\
& +9.1 \times 10^{-46} \frac{\mathrm{Jm}^{6}}{\mathrm{~s}} C(T)\left(\frac{T}{\mathrm{~K}}\right)^{-0.1687} \exp \left(-\frac{13179 \mathrm{~K}}{T}\right) n_{\mathrm{He}^{+}} n_{\mathrm{e}}^{2} \\
\Lambda_{\mathrm{He}^{+}} & =5.54 \times 10^{-30} \frac{\mathrm{Jm}^{3}}{\mathrm{~s}} C(T)\left(\frac{T}{\mathrm{~K}}\right)^{-0.397} \exp \left(-\frac{473638 \mathrm{~K}}{T}\right) n_{\mathrm{He}^{+}} n_{\mathrm{e}} .
\end{aligned}
$$

\subsubsection{Metals}

The $\mathrm{NC}^{2} \mathrm{M}$ incorporates fine structure line cooling of the elements $\mathrm{C}, \mathrm{N}, \mathrm{O}, \mathrm{Ne}, \mathrm{Mg}, \mathrm{Si}$, and $\mathrm{Fe}$ in the optically thin approximation. The total cooling due to metals reads

$\Lambda_{\mathrm{Z}}=\sum_{s} n_{s} \Lambda_{s}$

where the sum runs over all species $\mathrm{s}=\mathrm{C} \ldots \mathrm{C}^{5+}, \mathrm{N} \ldots \mathrm{N}^{6+}$, $\mathrm{O} \ldots \mathrm{O}^{7+}, \mathrm{Ne} \ldots \mathrm{Ne}^{9+}, \mathrm{Mg} \ldots \mathrm{Mg}^{11+}, \mathrm{Si} \ldots \mathrm{Si}^{13+}, \mathrm{Fe} \ldots \mathrm{Fe}^{25+}$. The cooling contribution of an individual species or ion is (the index $s$ is suppressed in the following)

$\Lambda=\sum_{i=1}^{L} p_{i} \sum_{j=1}^{i-1} \Delta E_{i j} A_{i j}$

for transitions $i \rightarrow j$ of the upper atomic energy level $i$ to the lower atomic energy level $j$ with energy separation $\Delta E_{i j}$ and with the Einstein coefficients $A_{i j}$ for spontaneous emission. The quantity $p_{i}$ is the population fraction, that is, the relative number of ions populating level $i$.

In order to compute the fractions $\left(p_{i}\right)_{i=1}^{L}$ for an ion, the statistical equilibrium equations are solved for a prescribed maximum number of atomic levels $L$ subject to the conservation condition $\sum_{i=1}^{L} p_{i}=1$. Different values for $L$ are allowed for different ions. It is assumed that excitation processes populating the

\footnotetext{
5 Generally, considering Spitzer (1978), the quantity $\alpha$ (the quotient $\chi_{1} / \phi_{1}$ of the functions $\chi_{1}$ and $\phi_{1}$ in Spitzer (1978)) is a slowly varying function of $T$ taking on values between 0.88 and 0.69 for $T$ between $100 \mathrm{~K}$ and $10^{5} \mathrm{~K}$. Here, following Cen (1992), a value of $\alpha=0.75$ is adopted.
} 
atomic states can be separated from those regulating the ionization state of an element, that is, that the calculation of the population fractions does not interfere with processes changing the ionization state. The principle of detailed balancing then reveals that the rate of ions leaving an atomic state is equal the rate of ions arriving at that state by (collisional) excitation and de-excitation processes and spontaneous emission (photoexcitation and stimulated emission are absent). Denoting the transition rate $i \rightarrow j$ as $Q_{i j}(i \neq j)$, it follows for any state $i$ that

$p_{i} \sum_{j=1 ; j \neq i}^{L} Q_{i j}=\sum_{j=1 ; j \neq i}^{L} p_{j} Q_{j i}, \quad i=1 \ldots L$,

or in vector notation, setting $Q_{i i}=-\sum_{j=1 ; j \neq i}^{L} Q_{i j}$ for convenience,

$\mathrm{Q}^{\top} \mathbf{p}=0$.

The constraint $\sum_{i=1}^{L} p_{i}=1$ is directly incorporated by exchanging with it the first equation of Eq. (46). Equation (47) is then replaced by

$\tilde{\mathrm{Q}} \mathbf{p}=(1,0, \ldots, 0)^{\top}, \quad \tilde{Q}_{i j}=\left\{\begin{array}{ll}1 & i=1 \\ \left(\mathrm{Q}^{\top}\right)_{i j} & i \neq 1\end{array}\right.$,

which can be solved for $\mathbf{p}$ with standard techniques. The transition rate is given by

$Q_{i j}=n_{e} Q_{i j}^{\mathrm{e}}+n_{\mathrm{H}} Q_{i j}^{\mathrm{H}}+n_{\mathrm{H}_{2}} Q_{i j}^{\mathrm{H}_{2}}+A_{i j}$,

where $Q_{i j}^{\mathrm{e}}\left(Q_{i j}^{\mathrm{H}}, Q_{i j}^{\mathrm{H}_{2}}\right)$ is the electron $\left(\mathrm{H}, \mathrm{H}_{2}\right)$ collisional (de)excitation rate. The Einstein coefficient $A_{i j}$ is defined to be zero for $i \leq j$. The excitation rate $(i<j)$ is related to the de-excitation rate $(i>j)$ according to

$Q_{i j}^{\mathrm{e}, \mathrm{H}, \mathrm{H}_{2}}=\left\{\begin{array}{lll}q_{i j}^{\mathrm{e}, \mathrm{H}, \mathrm{H}_{2}} & i<j & \text { (excitation) } \\ \frac{\omega_{j}}{\omega_{i}} \exp \left(\Delta E_{i j} / k T\right) q_{j i}^{\mathrm{e}, \mathrm{H}, \mathrm{H}_{2}} & i>j & \text { (de-excitation) }\end{array}\right.$

where $\omega_{i}\left(\omega_{j}\right)$ is the statistical weight of level $i(j)$. Collisions with neutral and molecular hydrogen is a relevant excitation mechanism only in the low-temperature regime when the electron density becomes small. The $\mathrm{NC}^{2} \mathrm{M}$ includes $\mathrm{H}$-excitation of lower lying fine-structure levels of the important coolants CI, CII, OI, SiII, and FeII and $\mathrm{H}_{2}$-excitation of CI and OI. The set of atomic transitions and the corresponding de-excitation rates are taken from Table 6 in Glover \& Jappsen (2007) and from Table B1 in Grassi et al. (2014).

Collisions by electrons is the dominant excitation process at higher temperatures. The electron excitation rate is given by

$q_{i j}^{\mathrm{e}}=2.172 \times 10^{-14} \frac{\mathrm{m}^{3}}{\mathrm{~s}}\left(\frac{13.61 \mathrm{eV}}{k T}\right)^{1 / 2} \exp \left(-\frac{\Delta E_{i j}}{k T}\right) \frac{\Upsilon_{i j}(T)}{\omega_{i}}$,

where $\Upsilon_{i j}(T)$ is the thermally-averaged collision strength for the $i \rightarrow j$ transition. To obtain $q_{i j}^{\mathrm{e}}$ the Chianti atomic database version 8 is consulted (Del Zanna et al. 2015). This database comprises critically evaluated atomic data like energy levels, radiation transition probabilities, statistical weights, and collision strength data for all metal ions considered here. In particular, Chianti samples $\Upsilon_{i j}$ in temperature applying the transition-type-dependent scaling of Burgess \& Tully (1992). This scaling allows us to accurately reproduce $\Upsilon_{i j}(T)$ over a large temperature range and guarantees a well-defined behavior in the limits $T \rightarrow 0, \infty$. In order to enable data access, an interface between the NIRVANA code and Chianti database has been designed. For each transition $i \rightarrow j$ a spline function is generated to represent $\Upsilon_{i j}(T)$ for all $T$. The spline-defining parameters are precomputed and stored for each transition, which permits a computationally cheap on-the-fly evaluation of $\Upsilon_{i j}(T)$.

The Chianti database is very comprehensive and contains many atomic fine-structure levels with a large number of radiative transitions. The volume of Chianti is utilized to study first the impact of the choice of uppermost atomic level $L$, which is used in the calculation of the ion population fractions, on the cooling rate. Computations are performed separately for each metal ion. Often, a fiducial value of $L=5$ is adopted, which might be insufficient to represent ion cooling rates accurately enough. The $L$-influence on the ion cooling rates, $\Lambda$, is illustrated in Fig. 1 as an example for the two ions OIII and FeVI. Figure 1 also shows this influence on the corresponding perion cooling coefficients, defined as $\Lambda / n_{\text {ion }} n_{\mathrm{e}}$, which in the case of electron-collision-dominated excitation are functions of temperature only and, thus, are particularly suited for tabulation (e.g., Oppenheimer \& Schaye 2013a). The temperature dependence $^{6}$ is displayed for $L=5$, for the maximum value, which is possible with Chianti ( $L=46$ for OIII, $L=96$ for FeVI) and for the default value used in the $\mathrm{NC}^{2} \mathrm{M}(L=11$ for OIII, $L=30$ for FeVI). Using five atomic levels, the cooling rate of both ions is off by an order of magnitude compared to the case exploiting the full Chianti database (dashed lines in Fig. 1). Deviations are much less (at most $40 \%$ ) for the $\mathrm{NC}^{2} \mathrm{M}$ default values, which were adopted as a trade-off between accuracy and computational costs. In summary, for many ions $L>5$ is required to ensure sufficient convergence of their cooling rates at all $T$. The recommended default values of $L$ are cataloged in Table 1 . This set represents about 2400 emission lines with photon energies ranging from $1.24 \times 10^{-4} \mathrm{eV}$ (IR) for the transition $2 s^{2} 2 p^{3}{ }^{2} P_{3 / 2} \rightarrow 2 s^{2} 2 p^{3}{ }^{2} P_{1 / 2}$ of NI to $8.26 \mathrm{keV}$ (X-ray) for the transition $3 p^{2} P_{3 / 2} \rightarrow 1 s^{2} S_{1 / 2}$ of FeXXVI.

Metal line cooling is a cost-intensive and complex part of the $\mathrm{NC}^{2} \mathrm{M}$. To further check its implementation certain spectral line intensity ratios for NII, OIII, and NeIII have been computed as a function of temperature in the vicinity of $T=10^{4} \mathrm{~K}$ for which theoretical estimates in the low-density limit are given in Osterbrock \& Ferland (2005):

$$
\begin{aligned}
& {[\mathrm{NII}] 2 s^{2} 2 p^{2}: \frac{I\left({ }^{1} D_{2} \rightarrow{ }^{3} P_{1}\right)+I\left({ }^{1} D_{2} \rightarrow{ }^{2} P_{1}\right)}{I\left({ }^{1} S_{0} \rightarrow{ }^{1} D_{2}\right)} \simeq \frac{8.23 \exp \left(2.5 \times 10^{4} \mathrm{~K} / T\right)}{1+4.4 \times 10^{-9} \frac{n_{\mathrm{e}}}{\mathrm{m}^{-3}}\left(\frac{T}{\mathrm{~K}}\right)^{-1 / 2}}} \\
& {[\mathrm{OIII}] 2 s^{2} 2 p^{2}: \frac{I\left({ }^{1} D_{2} \rightarrow{ }^{3} P_{1}\right)+I\left({ }^{1} D_{2} \rightarrow{ }^{2} P_{1}\right)}{I\left({ }^{1} S_{0} \rightarrow{ }^{1} D_{2}\right)} \simeq \frac{7.9 \exp \left(3.29 \times 10^{4} \mathrm{~K} / T\right)}{1+4.5 \times 10^{-10} \frac{n_{\mathrm{e}}}{\mathrm{m}^{-3}}\left(\frac{T}{\mathrm{~K}}\right)^{-1 / 2}}}
\end{aligned}
$$

$$
[\mathrm{NeIII}] 2 s^{2} 2 p^{4}: \frac{I\left({ }^{1} D_{2} \rightarrow{ }^{3} P_{1}\right)+I\left({ }^{1} D_{2} \rightarrow{ }^{2} P_{1}\right)}{I\left({ }^{1} S_{0} \rightarrow{ }^{1} D_{2}\right)} \simeq \frac{13.7 \exp \left(4.3 \times 10^{4} \mathrm{~K} / T\right)}{1+3.8 \times 10^{-11} \frac{n_{\mathrm{e}}}{\mathrm{m}^{-3}}\left(\frac{T}{\mathrm{~K}}\right)^{-1 / 2}} .
$$

The line intensity $I(i \rightarrow j) \propto p_{i} \Delta E_{i j} A_{i j}$. The outcome is depicted in Fig. 2. Despite the overall good agreement, the $\mathrm{NC}^{2} \mathrm{M}$ calculation is expected to be more precise since it uses temperature-dependent collision strengths whereas the theoretical expressions assume constant values.

\footnotetext{
6 The temperature range has been restricted to the regime where the ion fraction of the element is larger than $10^{-5}$. This is an optimization feature of the code and can be switched off. It utilizes the fact that for a small ion fraction neighboring ionization stages dominate the cooling of the element (see also Fig. 10, top panel).
} 
Table 1. Recommended numbers of atomic energy levels for metal cooling computations as adopted in the $\mathrm{NC}^{2} \mathrm{M}$ ( $z$ : ion charge).

\begin{tabular}{|c|c|c|c|c|c|c|c|c|c|c|c|c|c|c|c|c|c|c|c|c|c|c|c|c|c|c|}
\hline$z=$ & 0 & 1 & 2 & 3 & 4 & 5 & 6 & 7 & 8 & 9 & 10 & 11 & 12 & 13 & 14 & 15 & 16 & 17 & 18 & 19 & 20 & 21 & 22 & 23 & 24 & 25 \\
\hline $\mathrm{C}$ & 7 & 10 & 5 & 5 & 7 & 5 & & & & & & & & & & & & & & & & & & & & \\
\hline $\mathrm{N}$ & 5 & 10 & 10 & 5 & 5 & 7 & 5 & & & & & & & & & & & & & & & & & & & \\
\hline $\mathrm{O}$ & 5 & 7 & 11 & 10 & 5 & 5 & 7 & 5 & & & & & & & & & & & & & & & & & & \\
\hline $\mathrm{Ne}$ & - & 5 & 7 & 8 & 14 & 10 & 5 & 8 & 7 & 5 & & & & & & & & & & & & & & & & \\
\hline $\mathrm{Mg}$ & - & 5 & 7 & 5 & 7 & 8 & 14 & 10 & 5 & 8 & 7 & 5 & & & & & & & & & & & & & & \\
\hline $\mathrm{Si}$ & - & 10 & 5 & 5 & 15 & 5 & 8 & 8 & 14 & 10 & 5 & 8 & 8 & 7 & & & & & & & & & & & & \\
\hline $\mathrm{Fe}$ & - & 13 & 26 & 23 & 23 & 30 & 9 & 27 & 13 & 30 & 25 & 20 & 23 & 25 & 18 & 13 & 22 & 13 & 25 & 10 & 26 & 28 & 20 & 24 & 15 & 10 \\
\hline
\end{tabular}

Notes. Electron excitation data for NeI, MgI, SiI, and FeI is missing in Chianti.
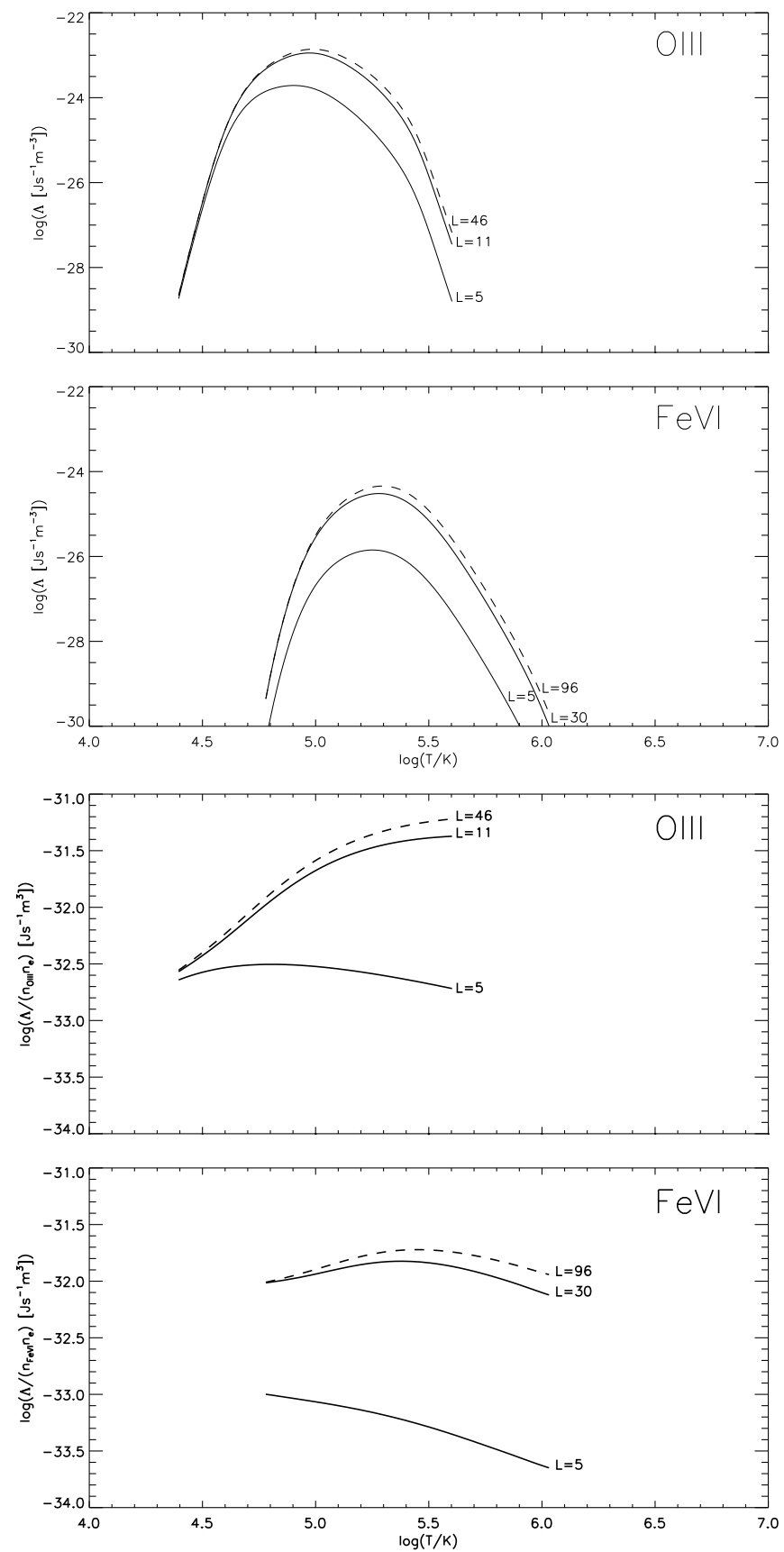

Fig. 1. Influence of parameter $L$ on the cooling rate (top panels) and on the per-ion cooling coefficient (bottom panels) for OIII and FeVI. The dashed line corresponds to the highest possible $L$ with Chianti. The results were obtained for an equilibrium model with $n_{\mathrm{H}}=10^{6} \mathrm{~m}^{-3}$.

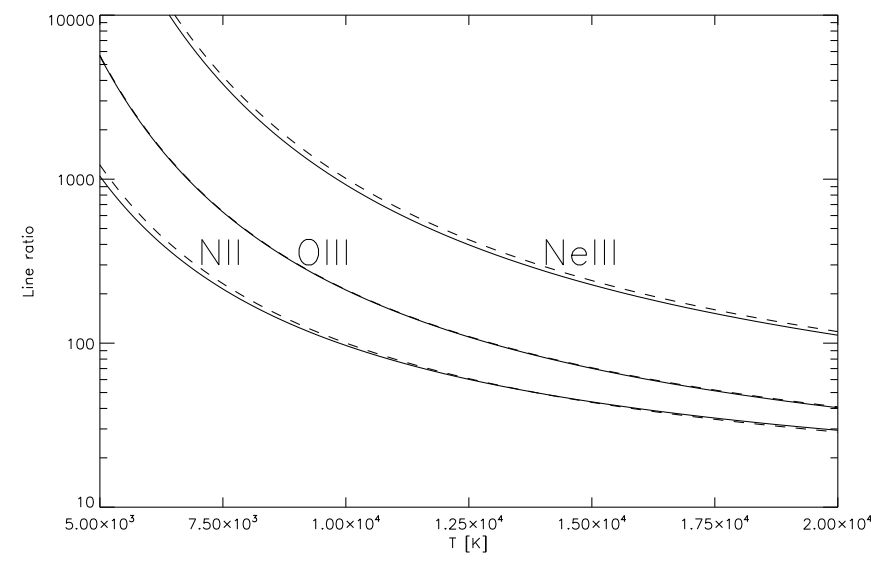

Fig. 2. Ratio of spectral line intensities obtained with the $\mathrm{NC}^{2} \mathrm{M}$ (solid lines) and theoretical estimates (dashed lines, Eqs. (52)-(54)) for $n_{\mathrm{H}}=$ $10^{4} \mathrm{~m}^{-3}$.

\subsection{Molecular cooling}

Molecular line radiation becomes an important gas coolant for temperatures below a few thousand Kelvin besides atomic fine structure cooling of $\mathrm{C}, \mathrm{O}, \mathrm{Si}$, and $\mathrm{Fe}$. Among the most relevant molecular coolants in the ISM are the molecules $\mathrm{H}_{2}, \mathrm{CO}, \mathrm{H}_{2} \mathrm{O}$, and $\mathrm{OH}$. In primordial or low-metallicity gas the hydrogen deuteride molecule, $\mathrm{HD}$, also contributes to gas cooling if sufficient amounts can be produced by chemical fractionation. The $\mathrm{NC}^{2} \mathrm{M}$ includes these five molecular coolants.

\subsection{1. $\mathrm{H}_{2}$}

For densities that are not too high $\left(n \lesssim 10^{9} \mathrm{~m}^{-3}\right)$ and for temperatures $T \approx 1000 \mathrm{~K}-4000 \mathrm{~K}$, molecular hydrogen cooling dominates other molecular cooling agents in the ISM. For higher densities and lower temperatures, $\mathrm{CO}$ cooling becomes equally important. To model $\mathrm{H}_{2}$ cooling I closely follow the work of Glover \& Abel (2008) who considered collisional excitation of rotovibrational levels for collision partners $\mathrm{H}, \mathrm{H}^{+}, \mathrm{He}, \mathrm{H}_{2}$, and $\mathrm{e}^{-}$. The ratio of ortho- $\mathrm{H}_{2}$ to para- $\mathrm{H}_{2}$ is fixed to $3: 1$ independent of temperature. The cooling rate in an optically thin environment can be written as

$\Lambda_{\mathrm{H}_{2}}=\frac{\Lambda_{\mathrm{H}_{2}, \mathrm{LTE}}}{1+\Lambda_{\mathrm{H}_{2}, \mathrm{LTE}} / \Lambda_{\mathrm{H}_{2}, 0}} n_{\mathrm{H}_{2}}$,

where $\Lambda_{\mathrm{H}_{2}, 0}$ denotes the sum of cooling rates per $\mathrm{H}_{2}$ molecule in the low-density limit due to the different collision partners:

$\Lambda_{\mathrm{H}_{2}, 0}=\sum_{s} \Lambda_{\mathrm{H}_{2}, s} n_{s}+\Lambda_{\mathrm{H}_{2}, \mathrm{H}^{+}, J=0 \leftrightarrow 1} n_{\mathrm{H}^{+}} \quad s=\mathrm{H}, \mathrm{H}^{+}, \mathrm{He}, \mathrm{H}_{2}, \mathrm{e}^{-}$. 
The individual contributions are

$\log \left(\frac{\Lambda_{\mathrm{H}_{2}, s}}{10^{-7} \mathrm{Jm}^{3} \mathrm{~s}^{-1}}\right)=\sum_{l=0}^{5} a_{s, l}\left(\log T_{3}\right)^{l}$,

with the fitting coefficients $a_{s, l}$ given in Table 8 of Glover \& Abel (2008) and $T_{3}=T / 10^{3} \mathrm{~K}$. Eq. (56) contains the special rate

$\Lambda_{\mathrm{H}_{2}, \mathrm{H}^{+}, J=0 \leftrightarrow 1}=4.76 \times 10^{-37} \frac{\mathrm{Jm}^{3}}{\mathrm{~s}}\left[2.25 \exp \left(-\frac{170.5 \mathrm{~K}}{T}\right)-0.75\right]$,

which separately accounts for the cooling due to proton collisional excitation of the rotational transition $J=0 \leftrightarrow 1$ associated with ortho-to-para state conversion, and this contribution is not implicit in the underlying fitting formula for $\Lambda_{\mathrm{H}_{2}, \mathrm{H}^{+}}$. Equation (58) leads to cooling or heating if the ortho-to-para ratio deviates from its thermal equilibrium value, which is the case here assuming a fixed 3:1 mix.

The function $\Lambda_{\mathrm{H}_{2} \text { LTE }}$ represents the cooling rate per $\mathrm{H}_{2}$ molecule when rotovibrational level populations are in local thermodynamic equilibrium (LTE) as expected at high gas densities. The temperature dependence of $\Lambda_{\mathrm{H}, \mathrm{LTE}}$ is approximated by the formula from Hollenbach \& McKee (1979),

$$
\begin{aligned}
\frac{\Lambda_{\mathrm{H}_{2} \mathrm{LTE}}}{\mathrm{Js}^{-1}}= & 9.5 \times 10^{-29} \frac{T_{3}^{3.76}}{1+0.12 T_{3}^{2.1}} \exp \left(-\frac{2.197 \times 10^{-3}}{T_{3}^{3}}\right) \\
& +3 \times 10^{-31} \exp \left(-\frac{0.51}{T_{3}}\right)+6.7 \times 10^{-26} \exp \left(-\frac{5.86}{T_{3}}\right) \\
& +1.6 \times 10^{-25} \exp \left(-\frac{11.7}{T_{3}}\right) .
\end{aligned}
$$

In the low-density limit, $\Lambda_{\mathrm{H}_{2}, \mathrm{LTE}} \gg \Lambda_{\mathrm{H}_{2}, 0}$, the effective cooling rate is $\Lambda_{\mathrm{H}_{2}} \rightarrow \Lambda_{\mathrm{H}_{2}, 0} n_{\mathrm{H}_{2}}$ whereas in the high-density limit $\Lambda_{\mathrm{H}_{2}}$ approaches $\Lambda_{\mathrm{H}_{2}, \mathrm{LTE}} n_{\mathrm{H}_{2}}$. At high density $\left(n \gtrsim 10^{16} \mathrm{~m}^{-3}\right)$ the gas starts to become opaque in the molecular line radiation. This is to the lowest order accounted for by multiplying $\Lambda_{\mathrm{H}_{2}}$ with the attenuation factor

$\tau_{\mathrm{H}_{2}}=\min \left[1,\left(\frac{n}{8 \times 10^{15} \mathrm{~m}^{-3}}\right)^{-0.45}\right]$

proposed by Ripamonti \& Abel (2004), and which depends only on the local density.

At even higher densities $\left(n \gtrsim 10^{20} \mathrm{~m}^{-3}\right)$ collision-induced emission (CIE) becomes a further important cooling effect. In the CIE process, deep collisions between $\mathrm{H}_{2}$ molecules or between $\mathrm{H}_{2}$ and $\mathrm{H}$ or He form a short-term supramolecular complex with an induced non-zero electric dipole. Such a configuration has a high probability of an energy transition followed by photon emission or absorption. According to Ripamonti \& Abel (2004), the CIE cooling rate is written as

$\Lambda_{\mathrm{CIE}, \text { thick }}=\Lambda_{\mathrm{CIE}} \min \left[1, \frac{1-e^{-\tau_{\mathrm{CIE}}}}{\tau_{\mathrm{CIE}}}\right]$,

where $\Lambda_{\mathrm{CIE}}$ is the cooling rate in the optically thin limit. The optical depth correction parameter $\tau_{\mathrm{CIE}}$ is given by

$\tau_{\mathrm{CIE}}=\left(\frac{n_{\mathrm{H}_{2}}}{7 \times 10^{21} \mathrm{~m}^{-3}}\right)^{2.8}$,
Table 2. Sources of $\tilde{\alpha}_{v}(T)$.

\begin{tabular}{llll}
\hline \hline Pair & $T(\mathrm{~K})$ & $v(\mathrm{THz})$ & Source $^{a}$ \\
\hline $\mathrm{H}_{2}-\mathrm{H}_{2}$ & $60-400$ & $0.3-495$ & Borysow (2002) \\
$\mathrm{H}_{2}-\mathrm{H}_{2}$ & $400-1000$ & $0.3-510$ & Borysow (2002) \\
$\mathrm{H}_{2}-\mathrm{H}_{2}$ & $1000-7000$ & $0.6-600$ & Borysow et al. (2001) \\
$\mathrm{H}_{2}-\mathrm{He}$ & $1000-7000$ & $0.75-603$ & Jorgensen et al. (2000) \\
$\mathrm{H}_{2}-\mathrm{H}$ & $1000-2500$ & $3-300$ & Gustafsson \& Frommhold (2003) \\
\hline
\end{tabular}

Notes. ${ }^{(a)}$ Data files at www . astro.ku.dk/ aborysow/programs.

to approximately account for cooling in the optically thick regime when $n \gtrsim 10^{22} \mathrm{~m}^{-3}$ (Hirano \& Yoshida 2013). Under the assumption of LTE, $\Lambda_{\mathrm{CIE}}$ is expressed by

$\Lambda_{\mathrm{CIE}}=\sum_{s} 4 \pi \int_{0}^{\infty} \alpha_{s, v}\left(T, n_{\mathrm{H}_{2}}, n_{s}\right) B_{v}(T) \mathrm{d} v, \quad s=\mathrm{H}_{2}, \mathrm{He}, \mathrm{H}$,

summing up cooling contributions for the collision pairs $\mathrm{H}_{2}-\mathrm{H}_{2}$, $\mathrm{H}_{2}-\mathrm{He}$, and $\mathrm{H}_{2}-\mathrm{H}$. The quantity $B_{v}(T)$ in units $\mathrm{Js}^{-1} \mathrm{~m}^{-2} \mathrm{~Hz}^{-1}$ denotes the black-body spectral radiance density (Planck's function) and $\alpha_{s, v}$, for each collision pair $s$, is the spectral collisioninduced absorption coefficient that depends on $T, n_{\mathrm{H}_{2}}$, and the number density $n_{s}$ of the respective collision partner. In order to perform the integral over frequency, $v$ data for $\alpha_{s, v}$ from various sources is used as listed in Table 2. These sources sample absorption coefficients per $\mathrm{H}_{2}$ molar density and per molar density of collision partner, $\tilde{\alpha}_{s, v}$ (in units $\mathrm{cm}^{-1} \mathrm{amg}^{-2}$ ), in the frequency interval and temperature range as specified in Table 2 for the different collision pairs. The specific $\tilde{\alpha}_{s, v}$ does not depend on the number densities of collision partners and is related to $\alpha_{s, v}$ (in units $\mathrm{m}^{-1}$ ) in Eq. (63) according to

$\alpha_{s, v}\left(T, n_{\mathrm{H}_{2}}, n_{s}\right)=1.3854 \times 10^{-49} n_{\mathrm{H}_{2}} n_{s} \tilde{\alpha}_{s, v}(T)$.

Equation (63) can then be rewritten as

$\Lambda_{\mathrm{CIE}}=\sum_{s} \Lambda_{\mathrm{CIE}, s} n_{s} n_{\mathrm{H}_{2}}, \quad s=\mathrm{H}_{2}, \mathrm{He}, \mathrm{H}$,

with frequency-integrated cooling rate coefficients $\Lambda_{\mathrm{CIE}, s}(T) \propto$ $\int \mathrm{d} v \tilde{\alpha}_{s, v}(T) B_{v}(T)$, which are only functions of temperature. The integration in $v$ is performed with the trapezoidal rule. Polynomial interpolation in $\log T$ is used to accurately represent those integrated rates as a function of temperature within the ranges given in Table 2, and a power-law extrapolation is adopted at higher temperatures for which no data exists:

$\log \left(\frac{\Lambda_{\mathrm{CIE}, s}}{\mathrm{Js}^{-1} \mathrm{~m}^{3}}\right)= \begin{cases}\sum_{l} a_{l}[\log (T / \mathrm{K})]^{l} & T<T_{\mathrm{u}} \\ b+c \log \left(T / T_{\mathrm{u}}\right) & T \geq T_{\mathrm{u}}\end{cases}$

The fitting coefficients $a_{l}, b, c, T_{\mathrm{u}}$ are summarized in Table 3 for the various collision pairs. The temperature dependence is shown in Fig. 3 demonstrating the quality of $\Lambda_{\mathrm{CIE}, s}$-fitting in the regimes where data is available. The approximation becomes uncertain for very low temperatures but the fitting formula can safely be applied.

\footnotetext{
$71 \mathrm{amg}$ (amagat) equals $44.615 \mathrm{~mol} \mathrm{~m}^{-3}$.
} 


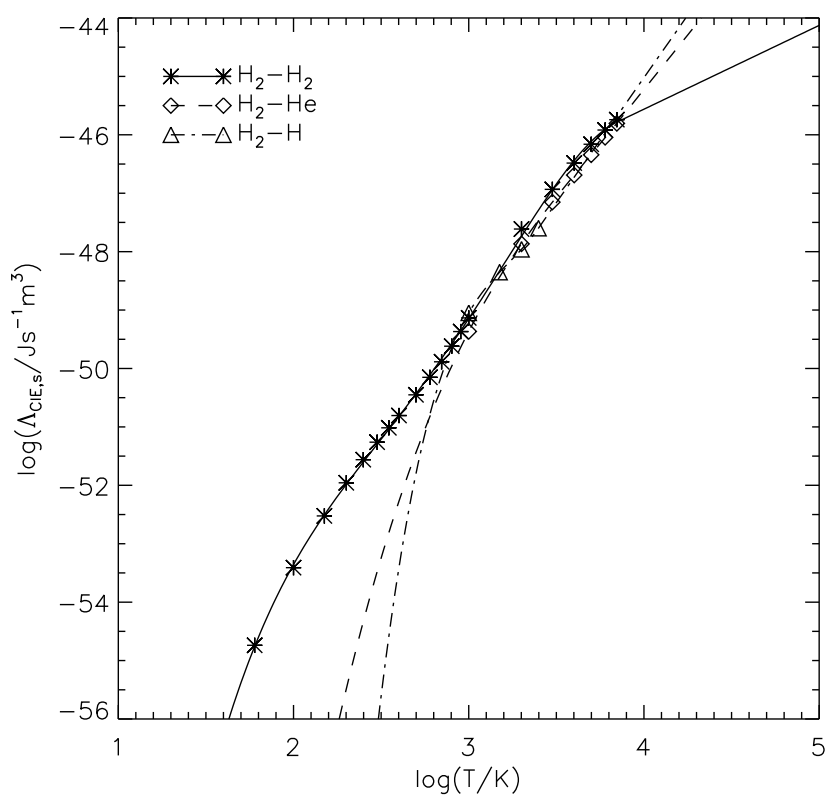

Fig. 3. CIE cooling fitting curves (lines) for the different collision pairs according to Eq. (66), and data points (symbols) derived from the sources in Table 2.

Table 3. Fitting coefficients for expression 66.

\begin{tabular}{llll}
\hline \hline & $\mathrm{H}_{2}-\mathrm{H}_{2}$ & $\mathrm{H}_{2}-\mathrm{He}$ & $\mathrm{H}_{2}-\mathrm{H}$ \\
\hline$a_{0}$ & -115.66396 & -136.66770 & -512.67285 \\
$a_{1}$ & 79.827549 & 66.217441 & 425.81532 \\
$a_{2}$ & -37.323660 & -16.976030 & -131.05073 \\
$a_{3}$ & 6.9829584 & 1.5345893 & 13.542074 \\
$a_{4}$ & -0.10236445 & & \\
$a_{5}$ & -0.071196266 & & \\
$b$ & -45.783785 & -45.802252 & -47.598415 \\
$c$ & 1.44 & 3.73 & 4.28 \\
$T_{\mathrm{u}}$ & $7000 \mathrm{~K}$ & $7000 \mathrm{~K}$ & $2500 \mathrm{~K}$ \\
\hline
\end{tabular}

\subsection{2. $H D$}

In primordial gas or low-metallicity gas, the HD molecule can be an important coolant at temperatures below a few hundred Kelvin and densities $n \gtrsim 10^{10} \mathrm{~m}^{-3}$ : a regime where $\mathrm{H}_{2}$ cooling becomes increasingly ineffective. HD cooling is represented in the $\mathrm{NC}^{2} \mathrm{M}$ by the cooling function of Lipovka et al. (2005). This cooling function is based on a detailed level population balance calculation covering rotovibrational transitions with $v \leq 3$ and $J \leq 8$, and applying excitation rates for collisions with $\mathrm{H}$.

Accordingly, the HD cooling rate is given by

$\Lambda_{\mathrm{HD}}=\tilde{\Lambda}_{\mathrm{HD}} n_{\mathrm{HD}}$

with the fitting function

$\log \left(\frac{\tilde{\Lambda}_{\mathrm{HD}}}{10^{-7} \mathrm{Js}^{-1}}\right)=\sum_{l, m=0}^{4} D_{l, m}\left[\log \left(\frac{n_{\mathrm{eff}}}{10^{6} \mathrm{~m}^{-3}}\right)\right]^{l}\left[\log \left(\frac{T}{\mathrm{~K}}\right)\right]^{m}$.

The coefficients $D_{l, m}$ are tabulated in Lipovka et al. (2005), Table 1. Since the excitation rate coefficients for HD-He collisions and $\mathrm{HD}-\mathrm{H}_{2}$ collisions are comparable to the excitation rate for HD-H collisions, as pointed out by Flower \& Le Bourlot (2000), an effective number density $n_{\mathrm{eff}}=n_{\mathrm{H}}+n_{\mathrm{H}_{2}}+n_{\mathrm{He}}$ is used in Eq. (68). Lipovka et al. (2005) emphasize the high accuracy of the above approximation for $100 \mathrm{~K} \lesssim T \lesssim 2 \times 10^{4} \mathrm{~K}$ and $10^{6} \mathrm{~m}^{-3} \lesssim n_{\text {eff }} \lesssim 10^{14} \mathrm{~m}^{-3}$. Within this density range the fitting function behaves well with respect to $T$ and can be safely applied outside of the prescribed temperature range. Outside the given density range, however, the fitting function is ill-conditioned. To circumvent mathematical problems, the HD cooling rate is therefore extended according to

$\Lambda_{\mathrm{HD}} \longrightarrow \begin{cases}\frac{n_{\mathrm{eff}}}{10^{6} \mathrm{~m}^{-3}} \Lambda_{\mathrm{HD}} & n_{\mathrm{eff}}<10^{6} \mathrm{~m}^{-3} \\ \Lambda_{\mathrm{HD}}\left(n_{\mathrm{eff}}=10^{14} \mathrm{~m}^{-3}\right) & n_{\mathrm{eff}}>10^{14} \mathrm{~m}^{-3},\end{cases}$

that is, it is linearly extrapolated down to zero density from the lower density bound, and it is assumed to be density-independent above the upper bound.

\subsection{3. $\mathrm{CO}$ and $\mathrm{H}_{2} \mathrm{O}$}

The $\mathrm{NC}^{2} \mathrm{M}$ implementation of $\mathrm{CO}$ cooling and $\mathrm{H}_{2} \mathrm{O}$ cooling is based on the work of Neufeld \& Kaufman (1993) and Neufeld et al. (1995) with some modifications as addressed in GFM10. The $\mathrm{CO}$ and $\mathrm{H}_{2} \mathrm{O}$ cooling rates are given by

$\Lambda_{s}=\left(\Lambda_{s, \text { rot }} n_{\mathrm{eff}, \mathrm{rot}, s}+\Lambda_{s, \mathrm{vib}} n_{\mathrm{eff}, \mathrm{vib}, s}\right) n_{s}, \quad s=\mathrm{CO}, \mathrm{H}_{2} \mathrm{O}$,

where $\Lambda_{s, \text { rot }}$ and $\Lambda_{s, \text { vib }}$ are cooling rate coefficients due to rotational and vibrational transitions, respectively, and $n_{\mathrm{eff}, \text { rot }, s}$, $n_{\text {eff,vib,s }}$ are effective number densities for collisions with $\mathrm{H}_{2}, \mathrm{H}$, and $\mathrm{e}^{-}$. In the Neufeld et al. papers, $n_{\mathrm{eff}, \text { rot }, s}=n_{\mathrm{eff}, \mathrm{vib}, s}=n_{\mathrm{H}_{2}}$ assuming $\mathrm{H}_{2}$ dominates collisional excitation of $\mathrm{CO}$ and $\mathrm{H}_{2} \mathrm{O}$. The cooling rate coefficients are determined from

$\frac{1}{\Lambda_{s, \mathrm{rot}}}=\frac{1}{\Lambda_{s, \mathrm{rot}, 0}}\left[1+\left(\frac{n_{\mathrm{eff}, \mathrm{rot}, s}}{n_{1 / 2, s}}\right)^{\alpha_{s}}\left(1-\frac{n_{1 / 2, s} \Lambda_{s, \mathrm{rot}, 0}}{\Lambda_{s, \mathrm{rot}, \mathrm{LTE}}}\right)\right]+\frac{n_{\mathrm{eff}, \mathrm{rot}, s}}{\Lambda_{s, \mathrm{rot}, \mathrm{LTE}}}$

for rotational transitions and from

$\frac{1}{\Lambda_{s, \mathrm{vib}}}=\frac{1}{\Lambda_{s, \mathrm{vib}, 0}}+\frac{n_{\mathrm{eff}, \mathrm{vib}, s}}{\Lambda_{s, \mathrm{vib}, \mathrm{LTE}}}$,

for vibrational transitions. Here, $\Lambda_{s, \text { rot }, 0}$ and $\Lambda_{s, \text { vib, } 0}$ denote the cooling coefficients in the low-density limit, $\Lambda_{s, \text { rot,LTE }}$ and $\Lambda_{s, \text { vib,LTE }}$ represent cooling rates per $s$ molecule in case the level population is in LTE, and $n_{1 / 2, s}$ is the effective density at which $\Lambda_{s, \text { rot }}=\Lambda_{s, \text { rot }, 0} / 2$. The low-density rate coefficients are pure functions of the temperature whereas $\Lambda_{s, \text { rot,LTE }}, \Lambda_{s \text {,vib,LTE }}, n_{1 / 2, s}$, and exponent $\alpha_{s}$ depend, in addition, on an optical depth variable $\tilde{N}_{s}$. This variable is defined by

$\tilde{N}_{s}=\frac{n_{s}}{|\nabla \cdot \mathbf{v}|}$

meaning an effective column density per unit velocity. The functions $\Lambda_{s \text {,rot,LTE}}, \Lambda_{s \text {,vib,LTE }}, n_{1 / 2, s}$, and $\alpha_{s}$ are sampled in Neufeld \& Kaufman (1993), Tables 2, 3, and 5, and Neufeld et al. (1995), Tables $1-3$, in $\left\{T, \log \tilde{N}_{s}\right\}$-space for the range

$$
\left(\frac{T}{\mathrm{~K}}\right) \times \log \left(\frac{\tilde{N}_{s}}{\frac{\mathrm{cm}^{-2}}{\mathrm{~km} \mathrm{~s}^{-1}}}\right)= \begin{cases}{[10,4000] \times[10,19]} & \mathrm{H}_{2} \mathrm{O}, \text { rot } \\ {[10,100] \times[14.5,19]} & \text { CO,rot } \\ {[100,2000] \times[14,19]} & \text { CO,rot } \\ {[100,4000] \times[13,20]} & \mathrm{H}_{2} \mathrm{O} / \mathrm{CO}, \text { vib }\end{cases}
$$

Linear interpolation within these tables is applied for function evaluation, and constant function continuation is adopted outside of the validated range. 
The expressions for the effective number densities are taken from GFM10 referring to the work of Yan (1997), Faure et al. (2004), and Meijerink \& Spaans (2005). Accordingly, for rotational cooling

$n_{\mathrm{eff}, \text { rot }, s}= \begin{cases}n_{\mathrm{H}_{2}}+10 n_{\mathrm{H}}+1291 n_{\mathrm{e}}\left(\frac{T}{\mathrm{~K}}\right)^{-1 / 2} & \\ \times 10^{15.749}\left(\frac{T}{\mathrm{~K}}\right)^{-1 / 6}-47.137\left(\frac{T}{\mathrm{~K}}\right)^{-1 / 3}+76.648\left(\frac{T}{\mathrm{~K}}\right)^{-1 / 2}-60.191\left(\frac{T}{\mathrm{~K}}\right)^{-2 / 3} & \mathrm{H}_{2} \mathrm{O} \\ n_{\mathrm{H}_{2}}+1.75 n_{\mathrm{H}}\left(\frac{T}{\mathrm{~K}}\right)^{1 / 4}+680 n_{\mathrm{e}}\left(\frac{T}{\mathrm{~K}}\right)^{-1 / 4} & \mathrm{CO},\end{cases}$

whereas for vibrational cooling

$n_{\mathrm{eff}, \mathrm{vib}, s}= \begin{cases}n_{\mathrm{H}_{2}}+10 n_{\mathrm{H}}+4.06 \times 10^{8} n_{\mathrm{e}} \frac{\exp \left(\frac{47.5}{(T / \mathrm{K})^{1 / 3}}\right)}{\sqrt{T / \mathrm{K}}} & \mathrm{H}_{2} \mathrm{O} \\ n_{\mathrm{H}_{2}}+50 n_{\mathrm{H}}+43 n_{\mathrm{e}}\left(\frac{T}{\mathrm{~K}}\right)^{0.938} \exp \left(\frac{68}{(T / \mathrm{K})^{1 / 3}}\right) & \mathrm{CO},\end{cases}$

\subsection{4. $\mathrm{OH}$}

Cooling of the $\mathrm{OH}$ molecule in the $\mathrm{NC}^{2} \mathrm{M}$ is based on the model developed in Hollenbach \& McKee (1979) for rotational cooling by molecules with dipole moment including optical depth effects and collisional de-excitation.

The $\mathrm{OH}$ cooling rate is written as

$\Lambda_{\mathrm{OH}}=\tilde{\Lambda}_{\mathrm{OH}} n_{\mathrm{OH}}$

Following Hollenbach \& McKee (1979), the cooling rate per $\mathrm{OH}$ molecule, $\tilde{\Lambda}_{\mathrm{OH}}$, is given by

$\tilde{\Lambda}_{\mathrm{OH}}=2.35 \times 10^{-26} \frac{\mathrm{J}}{\mathrm{s}}\left(\frac{T}{\mathrm{~K}}\right)^{2}\left[\frac{2+y_{\mathrm{m}}+0.6 y_{\mathrm{m}}^{2}}{1+c_{\tau}+n_{\mathrm{cr}} / n_{\mathrm{H}_{\mathrm{nuc}}}+1.5\left(n_{\mathrm{cr}} / n_{\mathrm{H}_{\mathrm{nuc}}}\right)^{0.5}}\right]$

where

$y_{\mathrm{m}}=\ln \left[1+\frac{c_{\tau}}{1+10 n_{\mathrm{cr}} / n_{\mathrm{H}_{\mathrm{nuc}}}}\right]$,

$n_{\mathrm{cr}}=1.5 \times 10^{16} \mathrm{~m}^{-3} \sqrt{\frac{T}{10^{3} \mathrm{~K}}}$,

and

$c_{\tau}=\frac{\tau_{T}\left[2 \pi \ln \left(2.13+\left(\tau_{T} / e\right)^{2}\right)\right]^{0.5}}{e^{-\tau_{\mathrm{d}}} /\left(1+\tau_{\mathrm{d}}^{2}\right)+2 \tau_{\mathrm{d}}\left[\ln \left(1+\tau_{T} / e\right) \ln \left(\tau_{T} / \tau_{\mathrm{d}} e\right)\right]^{0.5}}$.

The quantity $n_{\mathrm{cr}}$ denotes a critical density above which collisional de-excitation starts to become important and $c_{\tau}$ represents the optically thick regime. In the expression for $c_{\tau}$, the quantity $e$ is the Euler constant, and $\tau_{T}$ and $\tau_{\mathrm{d}}$ are the optical depths in the lines and due to dust absorption, respectively. In the one-cell numerical experiments below, $\mathrm{OH}$ cooling is considered in the optically thin limit, which is obtained by setting $\tau_{T}=\tau_{\mathrm{d}}=0$.

\subsection{Bremsstrahlung}

The $\mathrm{NC}^{2} \mathrm{M}$ takes into account plasma radiation losses in freefree transitions (Bremsstrahlung). A formula for the frequencyintegrated rate is given in Shapiro \& Kang (1987),

$\Lambda_{\mathrm{ff}}=1.426 \times 10^{-40} \frac{\mathrm{Jm}^{3}}{\mathrm{~s}} \sqrt{\frac{T}{\mathrm{~K}}} n_{\mathrm{e}} \sum_{i} z_{i}^{2} g_{\mathrm{ff}}\left(T, z_{i}\right) n_{i}$,

where the sum is over relevant ions with charge number $z_{i}$. The mean Gaunt factor, $g_{\mathrm{ff}}$, is given by

$g_{\mathrm{ff}}=\left\{\begin{array}{ll}0.79464+0.1243 \log \left(\frac{T}{\mathrm{~K}} z_{i}^{-2}\right) & T z_{i}^{-2}<3.2 \times 10^{5} \mathrm{~K} \\ 2.13164-0.1240 \log \left(\frac{T}{\mathrm{~K}} z_{i}^{-2}\right) & T z_{i}^{-2}>3.2 \times 10^{5} \mathrm{~K}\end{array}\right.$.

Bremsstrahlung losses become significant at $T \approx 10^{6} \mathrm{~K}$ where they compare with $\mathrm{H}$ and $\mathrm{He}$ atomic line cooling, and they dominate all metal cooling processes for $T \gtrsim 10^{7} \mathrm{~K}$. The main contribution comes from the ions $\mathrm{H}^{+}, \mathrm{He}^{+}$, and $\mathrm{He}^{++}$. In a fully ionized, solar abundance plasma, the high- $z$ metal ions of $\mathrm{O}, \mathrm{Ne}$, and $\mathrm{Fe}$ contribute to about another $10 \%$. Hence, those ions were included in the sum of Eq. (81).

\subsection{Cosmic ray heating}

The kinetic energy of the ejected electron in cosmic ray ionizations is partly converted into gas heating by Coloumb scattering, momentum transfer, and collisional de-excitation of excited $\mathrm{H}_{2}$ rotational states. The amount of energy deposited in the gas as heat is often assumed to be a constant fraction of the ejected electron energy. For instance, the models in GFM10 and RSO14 use a canonical value of $20 \mathrm{eV}$ per primary ionization. More generally, the heating fraction must be considered taking into account the ionization degree of the gas and its $\mathrm{H}-$ to- $\mathrm{H}_{2}$ abundance ratio. According to Dalgarno et al. (1999) the heating efficiency in a partly ionized $\mathrm{H}-\mathrm{H}_{2}-\mathrm{He}$ gas mixture can be represented by

$\eta=\frac{10 n_{\mathrm{H}_{2}} \eta_{\mathrm{H}_{2}-\mathrm{He}}+n_{\mathrm{H}} \eta_{\mathrm{H}-\mathrm{He}}}{10 n_{\mathrm{H}_{2}}+n_{\mathrm{H}}}$

where $\eta_{\mathrm{H}-\mathrm{He}}$ and $\eta_{\mathrm{H}_{2}-\mathrm{He}}$ are the efficiencies for separate $\mathrm{H}-\mathrm{He}$ and $\mathrm{H}_{2}-\mathrm{He}$ gas mixtures each with a $10 \%$ helium fraction in particle numbers. The individual efficiencies are given by the fitting formulae

$\eta_{\mathrm{H}-\mathrm{He}}=1-0.74\left[1+164.8\left(n_{\mathrm{e}} / n_{\mathrm{H}}\right)^{0.984}\right]^{-1}$

$\eta_{\mathrm{H}_{2}-\mathrm{He}}=1-0.9\left[1+16.11\left(n_{\mathrm{e}} / n_{\mathrm{H}_{2}}\right)^{0.515}\right]^{-1}$,

and they are accurate to within $10 \%$ to the Monte-Carlo simulation results of Dalgarno et al. (1999) up to moderate gas ionization levels, $n_{\mathrm{e}} / n_{\mathrm{H}} \lesssim 0.1$ or $n_{\mathrm{e}} / n_{\mathrm{H}_{2}} \lesssim 0.1$, respectively.

In the $\mathrm{NC}^{2} \mathrm{M}$ the total heating rate for a mean ejected electron energy of $30 \mathrm{eV}$ due to $\mathrm{H}$ and $\mathrm{H}_{2}$ primary cosmic ray ionizations is then expressed by

$\Gamma_{c r}=(30 \mathrm{eV}) \eta\left(\xi_{\mathrm{H}} n_{\mathrm{H}}+\xi_{\mathrm{H}_{2}} n_{\mathrm{H}_{2}}\right)$.

To give some numbers, for a neutral $\mathrm{H}-\mathrm{He}\left(\mathrm{H}_{2}-\mathrm{He}\right)$ gas mixture one obtains a heating efficiency of $\eta=0.26(\eta=0.1)$ corresponding to a mean energy per cosmic ray ionization of $7.8 \mathrm{eV}$ $(3 \mathrm{eV})$. The presence of free electrons substantially increases the energy loss of the ejected electron by Coloumb scattering. For a partly ionized $\mathrm{H}-\mathrm{He}\left(\mathrm{H}_{2}-\mathrm{He}\right)$ gas mixture with $n_{\mathrm{e}}=0.1 n_{\mathrm{H}}$ $\left(n_{\mathrm{e}}=0.1 n_{\mathrm{H}_{2}}\right)$, the heating efficiency grows to $\eta=0.96(\eta=$ $0.85)$ corresponding to a mean energy per cosmic ray ionization of $28.8 \mathrm{eV}(25.5 \mathrm{eV})$. 


\section{Equilibrium chemistry and cooling}

The $\mathrm{NC}^{2} \mathrm{M}$ has been developed with the ultimate aim of following non-equilibrium chemistry in multidimensional magnetohydrodynamics applications. However, it is essential to first check the implementation by considering chemical equilibrium models. To find the chemical equilibrium for the network specified in Tables A.1-A.3 at a fixed temperature, density, and elemental composition the numerical solver as described in Ziegler (2016) is used. The system of kinetic rate equations is evolved for a sufficiently long time until the solution has converged close to chemical equilibrium, which is defined by a relative residual of $10^{-6}$. The equilibrium species abundances are calculated as a function of temperature in the range $10 \mathrm{~K} \leq T \leq 10^{8} \mathrm{~K}$ at 801 nodes with $\Delta \log T=8.75 \times 10^{-3}$ for three different densities $n_{\mathrm{H}}=10^{4} \mathrm{~m}^{-3}, 10^{6} \mathrm{~m}^{-3}, 10^{10} \mathrm{~m}^{-3}$ in order to cover ISM conditions from dilute to dense gas. At this point we recall that in the presence of an UV background, dilute gas is not shielded from photoionizing or photodissociating radiation, which certainly would have a profound influence on the low-temperature equilibrium balance obtained here. The calculations assume an elemental composition given by default solar abundances as cataloged in Grevesse \& Sauval 1998. This results in a total metallicity of $Z=0.96 Z_{\odot}$. Other metals missing in the $\mathrm{NC}^{2} \mathrm{M}$ make a contribution of $0.04 Z_{\odot}$.

In order to illustrate the potential importance of grain-surface catalysis and cosmic ray ionization effects, equilibrium computations are performed for the full chemical network including dust and cosmic rays as well as for reduced networks neglecting either the dust component or the cosmic ray component. A pure gas-phase network is also considered ignoring both dust and cosmic rays. Variations in metallicity are not investigated. In the models including cosmic ray reactions, a canonical cosmic ray ionization rate of $\xi_{\mathrm{H}}=2.5 \times 10^{-17} \mathrm{~s}^{-1}$ is adopted. For grain-surface reactions, the dust temperature, $T_{\mathrm{d}}$, and the parameter $G$ appearing in expression (23) for grain charging need to be defined. As in RSO14 we adopt a value of $T_{\mathrm{d}}=10 \mathrm{~K}$ since the rate coefficient given by Eq. (17) is not very sensitive to the dust temperature in the regime $6 \mathrm{~K} \lesssim T_{\mathrm{d}} \lesssim 50 \mathrm{~K}$. As far as concerns grain charging, we set $G \equiv 0$ consistent with the limiting case of no radiation, that is, no photoelectric charging effect.

\subsection{Molecule abundances}

Equilibrium abundances for the three important molecular coolants $\mathrm{H}_{2}, \mathrm{CO}$, and $\mathrm{H}_{2} \mathrm{O}$ and for the further molecules $\mathrm{O}_{2}$ and $\mathrm{OH}$ are shown in Fig. 4 as a function of temperature for different gas densities (different panels in a row) and for the considered chemical network variants (different line styles of curves). Molecular fractions have been normalized to the $\mathrm{H}$ nuclei density in the case of $\mathrm{H}_{2}, \mathrm{C}$ nuclei density in the case of $\mathrm{CO}$, and $\mathrm{O}$ nuclei density in the case of $\mathrm{H}_{2} \mathrm{O}, \mathrm{O}_{2}$, and $\mathrm{OH}$. Only the low-temperature regime is shown since molecules are effectively absent at $T \gtrsim 2 \times 10^{4} \mathrm{~K}$ irrespective of density.

Molecular abundances for the full network case are depicted as solid curves in Fig. 4. It is first noted that the obtained $\mathrm{H}_{2}$ fraction is larger than in RSO14 because dust-catalytic $\mathrm{H}_{2}$ formation is more effective due to the higher dust total cross section used in our standard model. However, when assuming identical dust parameters, excellent agreement can be found between RSO14 and the full network case. The resulting $\mathrm{CO}$ distribution also matches well with that in RSO14 under this assumption ${ }^{8}$. At

8 There are still minor discrepancies probably caused by some differences in the rate coefficients. In particular, if we drop the $T^{-1 / 2}$ - moderate densities the curves of the $\mathrm{O}_{2}$ and $\mathrm{OH}$ fractions closely follow that of $\mathrm{H}_{2} \mathrm{O}$. The $\mathrm{H}_{2}, \mathrm{CO}$, and $\mathrm{H}_{2} \mathrm{O}$ fractions generally grow with increasing density.

At the highest density, $n_{\mathrm{H}}=10^{10} \mathrm{~m}^{-3}$, most hydrogen is in molecular form and the $\mathrm{H}_{2}$ fraction approaches unity up to a temperature of $\sim 2500 \mathrm{~K}$, and rapidly drops hereafter. The weakly visible cut-off near this temperature is attributed to the functional form of coefficient Eq. (17) for dust-surface catalysis of $\mathrm{H}_{2}$. This cut-off is also striking as sharp transitions in the other molecules, since $\mathrm{H}_{2}$ largely controls their chemistry. The $\mathrm{CO}$ fraction is close to unity for $T \lesssim 500 \mathrm{~K}$ and in the window $2500 \mathrm{~K} \lesssim T \lesssim 4000 \mathrm{~K}$. In between, $500 \mathrm{~K} \lesssim T \lesssim 2500 \mathrm{~K}$, the CO fraction decreases to $\sim 10^{-2}$ associated with the increase in $\mathrm{H}_{2} \mathrm{O}$ fraction. Evidently, more oxygen becomes bound in $\mathrm{H}_{2} \mathrm{O}$, which is no longer available to form $\mathrm{CO}$. Also, the formation of molecular oxygen is largely suppressed in the temperature regime $300 \mathrm{~K} \lesssim T \lesssim 2500 \mathrm{~K}$ in favor of the synthesis of water.

The absence of cosmic ray reactions has a paramount influence on the chemical equilibrium abundances since cosmic rays as the dominant driver for ionization at lower temperature ceases, and the ionization balance is determined by collisional effects and neutralization of ions on dust grains only. This results in a very low level of ionization and the chemistry is controlled by neutral-neutral reactions and $\mathrm{H}_{2}$ formation on grains. Abundances for the CR-free network are depicted as dash-dotted curves in Fig. 4. Up to a temperature of $\sim 4000 \mathrm{~K}(\sim 3300 \mathrm{~K}$ for $n_{\mathrm{H}}=10^{10} \mathrm{~m}^{-3}$ ) hydrogen is predominantly bound in $\mathrm{H}_{2}$ followed by a smaller fraction bound in $\mathrm{H}_{2} \mathrm{O}$. The increased amount of $\mathrm{H}_{2}$ compared to the full network case is the result of missing cosmic ray ionization and CR-induced photodissociation of $\mathrm{H}_{2}$. The fraction of atomic hydrogen is very low in this temperature regime: $n_{\mathrm{H}} / n_{\mathrm{H}_{\mathrm{nuc}}}<10^{-6}\left(<10^{-8},<10^{-12}\right)$ at $n_{\mathrm{H}}=10^{4} \mathrm{~m}^{-3}$ $\left(n_{\mathrm{H}}=10^{6} \mathrm{~m}^{-3}, n_{\mathrm{H}}=10^{10} \mathrm{~m}^{-3}\right)$ compared to $n_{\mathrm{H}} / n_{\mathrm{H}_{\text {nuc }}}>0.8$ $\left(>0.18,>5 \times 10^{-5}\right)$ in the full network case. Likewise, carbon is almost completely bound in $\mathrm{CO}$ with a fraction close to unity below $T \approx 5000 \mathrm{~K}\left(\approx 4500 \mathrm{~K}\right.$ for $\left.n_{\mathrm{H}}=10^{10} \mathrm{~m}^{-3}\right)$. Other C-bearing molecules like $\mathrm{CH}_{2}$, for example, exist only in small amounts with a fraction of $n_{\mathrm{CH}_{2}} / n_{\mathrm{C}_{\text {nuc }}}<10^{-10}$ for this molecule. There are three distinct temperature ranges where the $\mathrm{H}_{2} \mathrm{O}$ fraction is nearly constant. At a density $n_{\mathrm{H}}=10^{4} \mathrm{~m}^{-3}$ the intervals are $T \lesssim 220 \mathrm{~K}, 250 \mathrm{~K} \lesssim T \lesssim 1000 \mathrm{~K}$, and $1100 \mathrm{~K} \lesssim T \lesssim 4000 \mathrm{~K}$, respectively. The intervals slightly change with density, and for the highest density the first two temperature regimes seem to merge. The $\mathrm{O}_{2}$ fraction drops by many order of magnitudes in the third temperature regime where the $\mathrm{H}_{2} \mathrm{O}$ fraction reaches its largest value. This $\mathrm{O}_{2}$ depletion effect is also seen in the highdensity full network case but in the CR-free network it occurs at all densities.

Equilibrium abundances for the dust-free network are depicted as dashed curves in Fig. 4. The absence of dust-assisted reactions, in particular the catalysis of $\mathrm{H}_{2}$, has a substantial impact on the $\mathrm{H}_{2}$ abundance, and therefore on the overall molecular chemistry. Molecular fractions are substantially reduced at lower temperatures compared to the full network case except at the highest density where three-body reactions contribute to $\mathrm{H}_{2}$ formation. Therefore, despite the lack of dust, the $\mathrm{H}_{2}$ fraction at $n_{\mathrm{H}}=10^{10} \mathrm{~m}^{-3}$ is roughly one order of magnitude larger than for the other densities for $T \lesssim 2500 \mathrm{~K}$. Compared with the full network case, the buckle in $\mathrm{CO}$ around $1000 \mathrm{~K}$ is absent, and

dependence from $k_{506}$ of the important reaction 506 (this dependence is somewhat hidden in the reference Petuchowski et al. 1989), excellent agreement is found as well. 

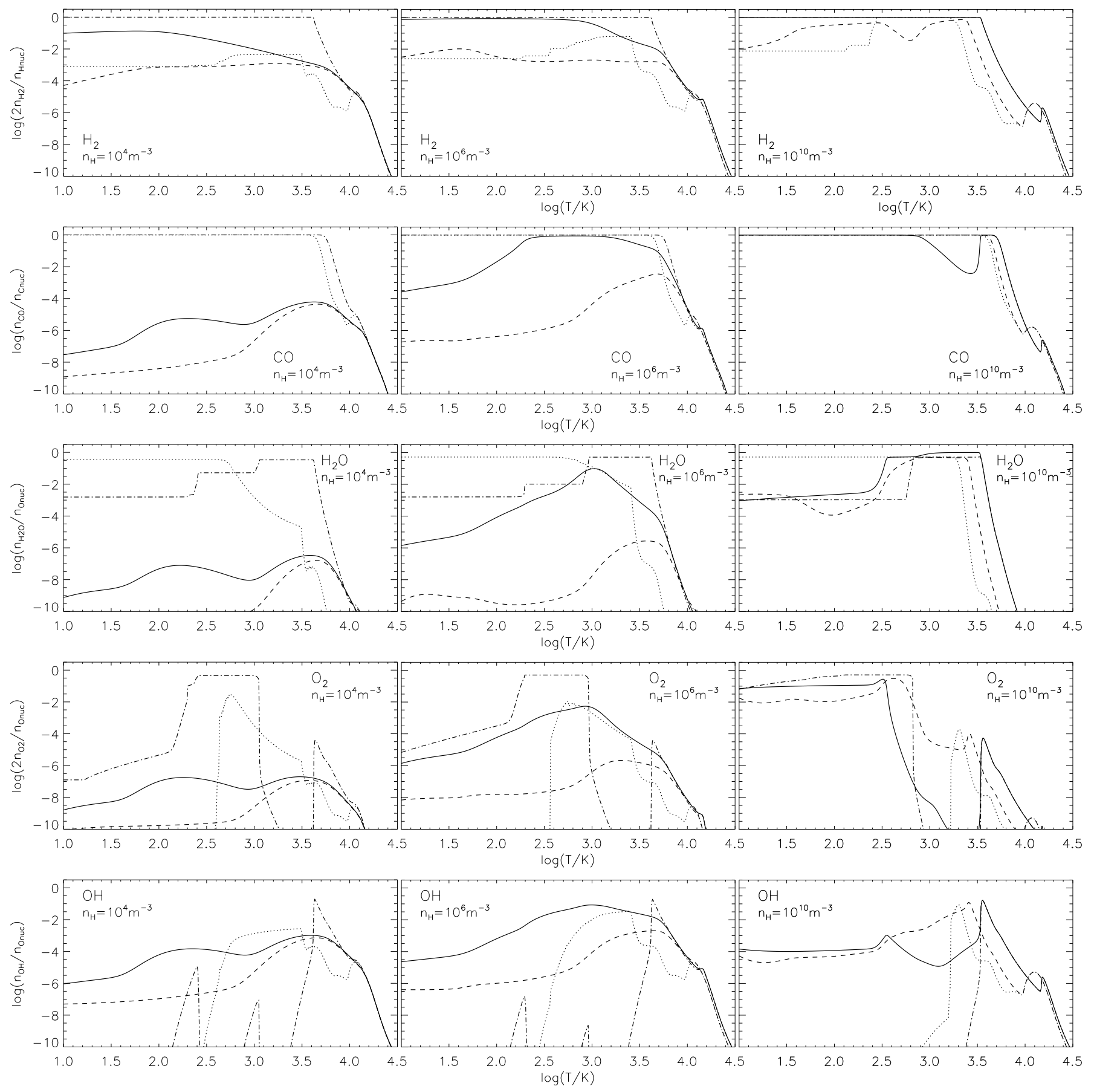

Fig. 4. Fractions of $\mathrm{H}_{2}$ (first row), $\mathrm{CO}$ (second row), $\mathrm{H}_{2} \mathrm{O}$ (third row), $\mathrm{O}_{2}$ (fourth row), and $\mathrm{OH}$ (fifth row) for different densities (from left to right). Equilibrium solutions for the full network (solid), dust-free network (dashed), CR-free network (dash-dotted), and pure gas-phase network (dotted) are shown.

a substantial amount of $\mathrm{H}_{2} \mathrm{O}\left(\mathrm{O}_{2}\right)$ is present in the vicinity of $1000 \mathrm{~K}(400 \mathrm{~K})$.

Abundances for the pure gas-phase network, which ignores both dust and cosmic rays, are depicted as dotted curves in Fig. 4. At moderate densities and below a temperature of $\sim 1000 \mathrm{~K}$ the $\mathrm{H}_{2}$ fraction is up to two orders of magnitude smaller than in the full network case. At the same time the $\mathrm{H}_{2} \mathrm{O}$ fraction is larger by up to several orders of magnitude, and is nearly constant. The $\mathrm{CO}$ fraction is close to one up to $T \approx 5000 \mathrm{~K}$ and a somewhat smaller temperature at the highest density. The buckle in the $\mathrm{CO}$ distribution most prominent in the high-density full network case vanishes since its appearance is tightly correlated with a simultaneous high $\mathrm{H}_{2} \mathrm{O}$ fraction. Below a certain temperature both $\mathrm{O}_{2}$ and $\mathrm{OH}$ get substantially depleted independent of density because most oxygen is bound in $\mathrm{H}_{2} \mathrm{O}$.

\subsection{Ionization structure}

The ionization structure is illustrated in Figs. 5-8 as examples for the metal elements $\mathrm{C}, \mathrm{O}, \mathrm{Si}$, and $\mathrm{Fe}$. As expected, the ionization fractions in the high-temperature regime are pure functions of the temperature and are independent of density. Ion recombination on dust and cosmic ray processes has a negligible effect at higher temperatures, and the ionization fraction is 


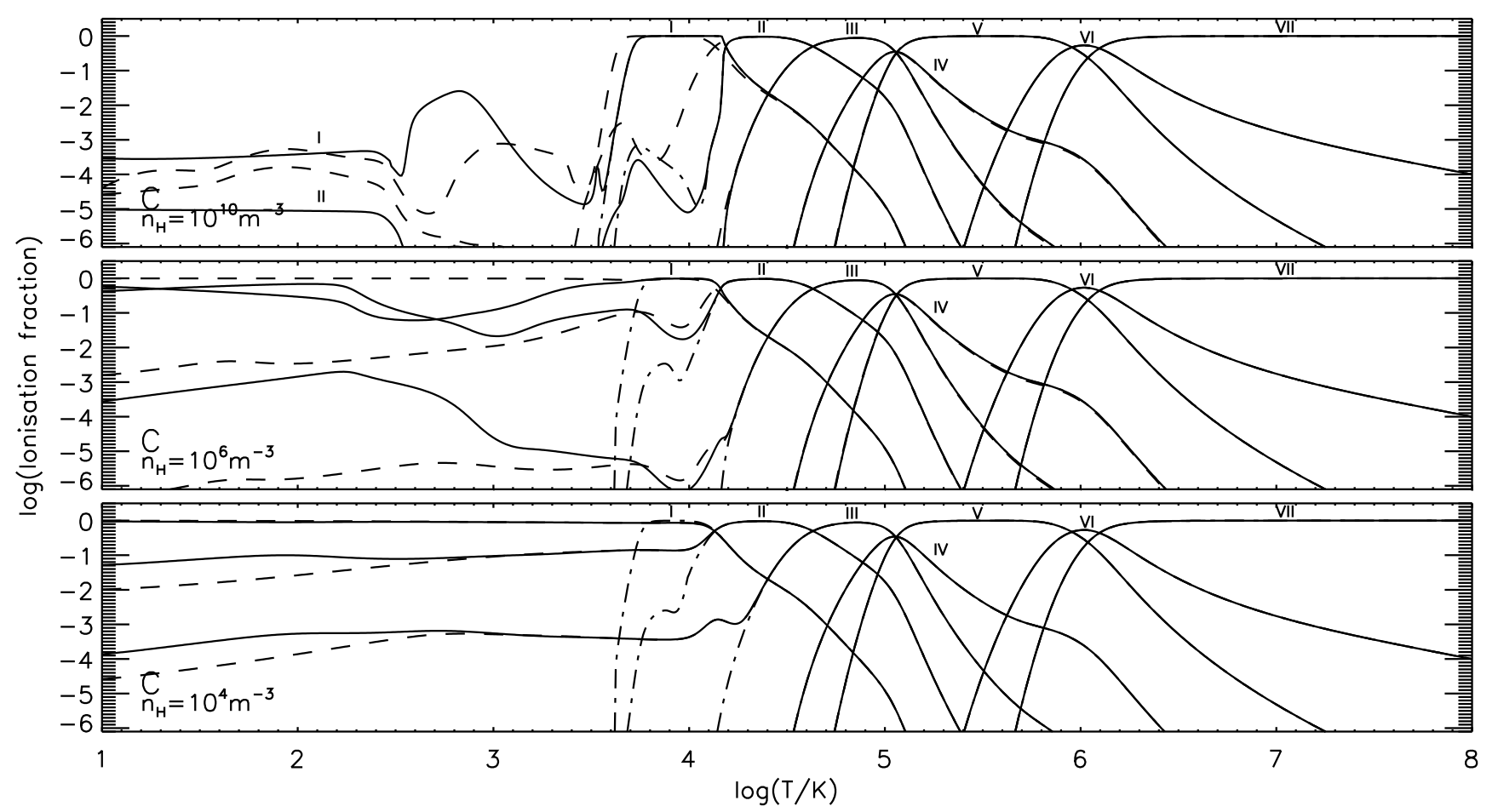

Fig. 5. Ionization structure of $\mathrm{C}$ for different densities. Equilibrium solution for the full network (solid), dust-free network (dashed), and CR-free network (dash-dotted).

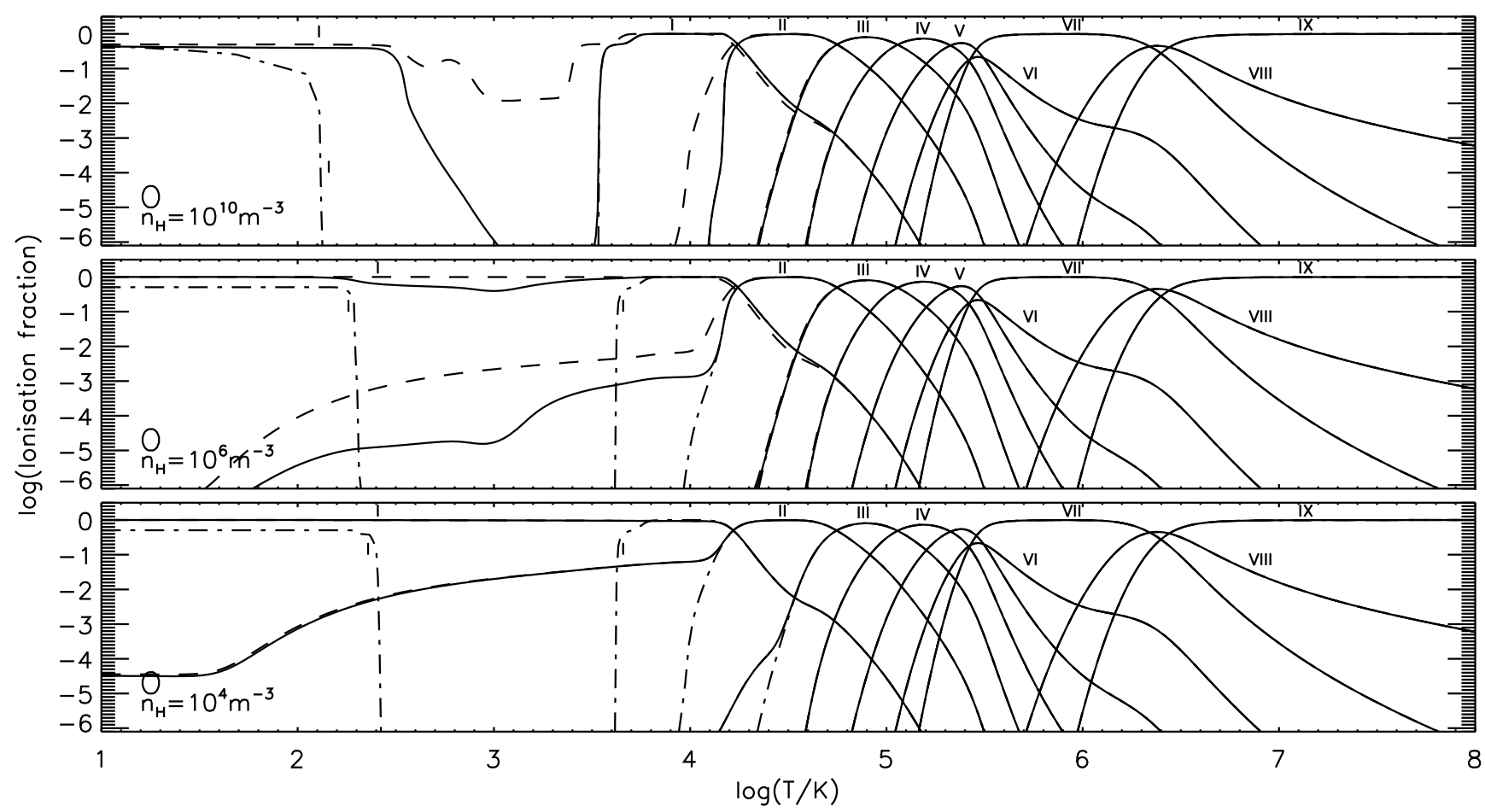

Fig. 6. Analog to Fig. 5 for O.

solely determined by the balance between electron collisional ionization, electron-ion recombination, and, to a lesser extent, charge transfer process. The high-temperature ionization structure, in particular the shapes and peak positions of the individual ionization state curves, is in excellent agreement with previous literature results, such as Sutherland \& Dopita (1993) or Gnat \& Sternberg (2007).
At temperatures $T \lesssim 10^{4} \mathrm{~K}$ the lower ionization stages of metal elements show an explicit dependence of their fractions on the gas density, dust content, and cosmic ray strength. In the absence of cosmic rays (dash-dotted lines) the fractions of SilI and FeII, for instance, are substantially reduced compared to the full network case (solid lines) where they are close to unity except for the highest density. The overall impact of dust 


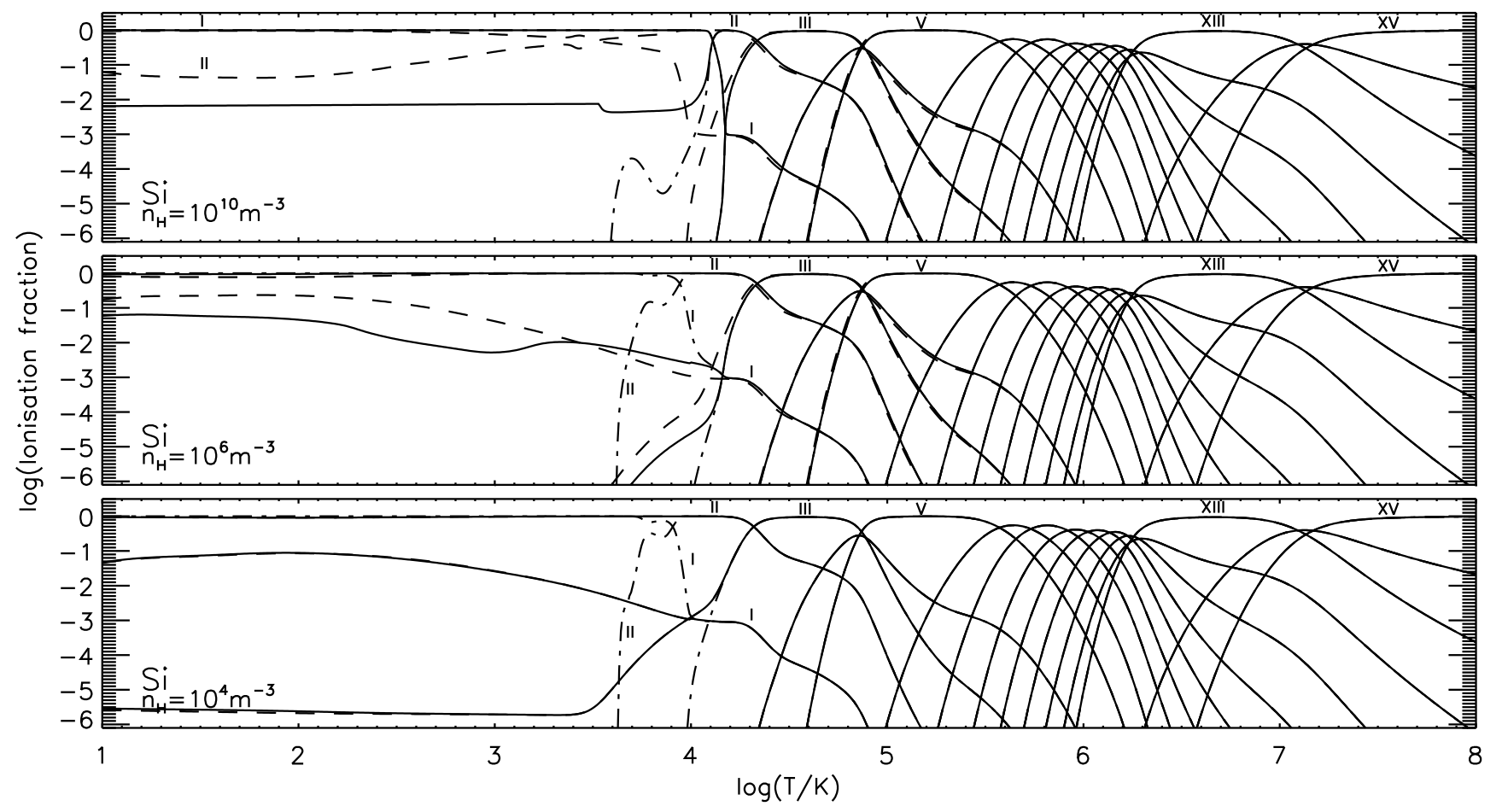

Fig. 7. Analog to Fig. 5 for Si.

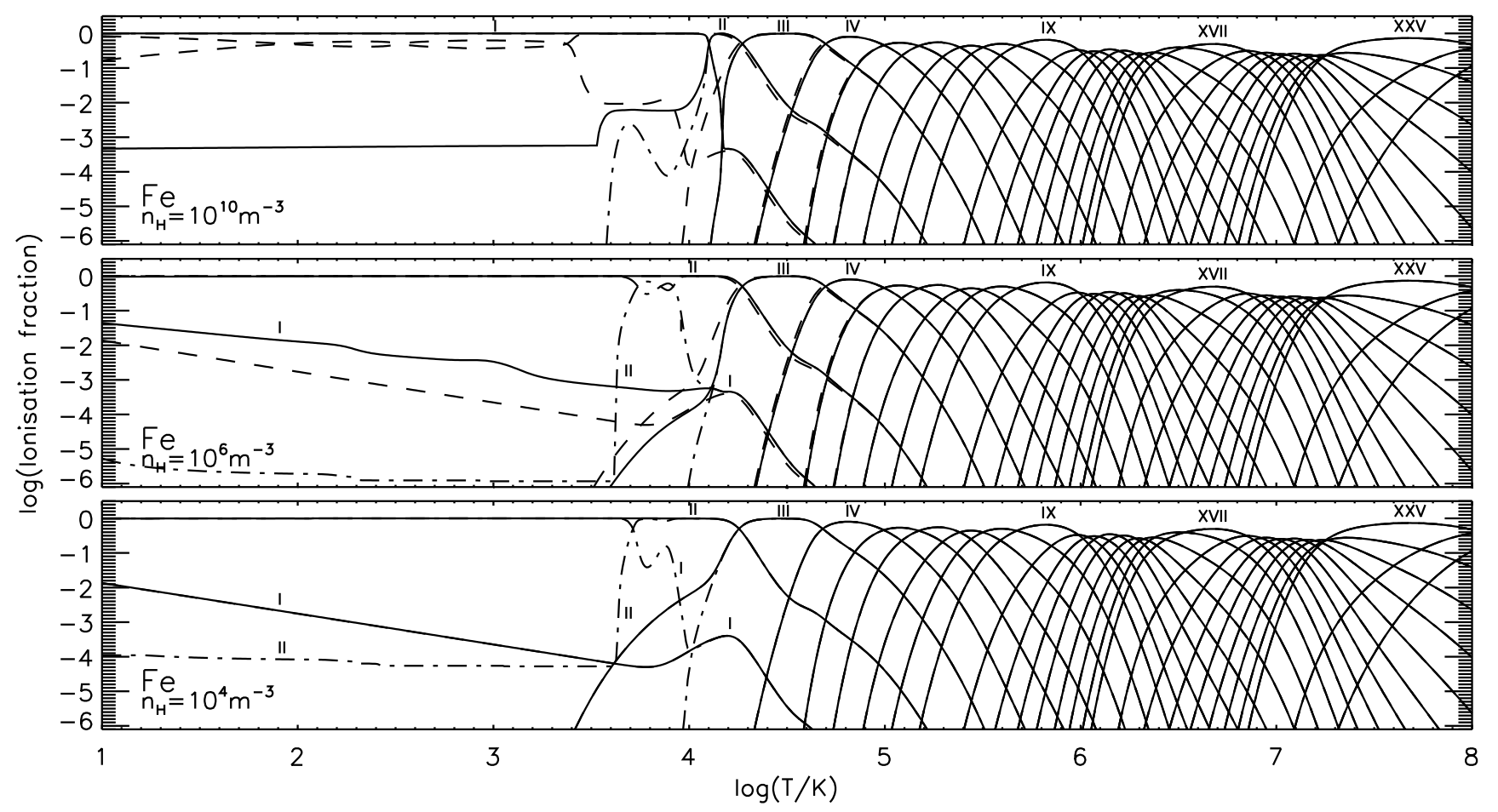

Fig. 8. Analog to Fig. 5 for Fe.

is less pronounced at lower densities but becomes apparent for $n_{\mathrm{H}}=10^{10} \mathrm{~m}^{-3}$. For instance, the fraction of FeII grows by three orders of magnitude in the absence of dust (dashed lines) from $\sim 4 \times 10^{-4}$ to a value of $\sim 0.3$. Generally, the CI and OI abundance is heavily influenced by molecular reactions taking place in the low-temperature regime. This is prominent in CI at $n_{\mathrm{H}}=10^{10} \mathrm{~m}^{-3}$ where a significant amount of carbon is bound in CO. Molecule formation of $\mathrm{O}_{2}$ and $\mathrm{H}_{2} \mathrm{O}$ leads to the broad gap in the abundance of OI around $T=1000 \mathrm{~K}$ especially when cosmic rays are absent.

\subsection{Cooling}

In this section cooling functions computed under the assumption of chemical equilibrium are presented. For convenience, the functions are normalized to the $\mathrm{H}$ nuclei number density 


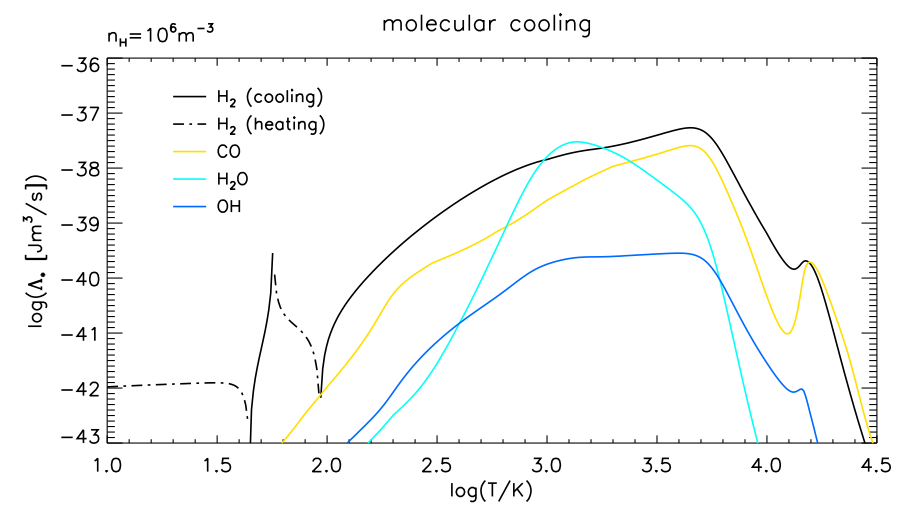

Fig. 9. Molecular cooling in chemical equilibrium for the full network.

squared, $\Lambda_{*}=\Lambda / n_{\mathrm{H}_{\text {nuc }}}^{2}\left(\Gamma / n_{\mathrm{H}_{\text {nuc }}}^{2}\right.$ for heating terms, $\Lambda_{\text {net }} / n_{\mathrm{H}_{\text {nuc }}}^{2}$ for net cooling), where $\Lambda_{*}$ has units $\mathrm{J} \mathrm{m}^{3} \mathrm{~s}^{-1}\left(=10^{13} \mathrm{erg} \mathrm{cm}^{3} \mathrm{~s}^{-1}\right)$. Calculations are shown for the full network at a density of $n_{\mathrm{H}}=10^{6} \mathrm{~m}^{-3}$ if not otherwise stated.

Figure 9 shows equilibrium cooling functions for the molecules $\mathrm{H}_{2}, \mathrm{CO}, \mathrm{H}_{2} \mathrm{O}$, and $\mathrm{OH}$ resulting from line cooling. The $\mathrm{H}_{2}$ cooling function, in addition, contains contributions from reaction kinetics (dissociation cooling and formation heating). Also, CIE cooling is included in the $\mathrm{H}_{2}$ function which is, however, unimportant at the gas densities explored here. The $\mathrm{H}_{2}$ cooling function is negative for $T \lesssim 40 \mathrm{~K}$ and between $T \approx 60 \mathrm{~K}$ and $T \approx 100 \mathrm{~K}$, that is, there is net heating indicated by dashdotted lines in Fig. 9. The heating below $40 \mathrm{~K}$ is caused by chemical heating mainly due to $\mathrm{H}_{2}$ formation on dust grains. The second heating window is a consequence of fixing the ortho-topara $\mathrm{H}_{2}$ ratio to $3: 1$, which results in heating (or cooling) below (or above) $T \approx 155 \mathrm{~K}$ according to Eq. (58). This process interleaves with the other thermal processes and leads to net heating in this temperature interval. If Eq. (58) were skipped, the second heating window would vanish. $\mathrm{H}_{2}$ cooling dominates molecular cooling except in the vicinity of $T \approx 1000 \mathrm{~K}$ where cooling due to $\mathrm{H}_{2} \mathrm{O}$ peaks, and above $T \approx 6000 \mathrm{~K}$ where $\mathrm{CO}$ significantly contributes to molecular cooling. The cooling due to $\mathrm{OH}$ is more than one order of magnitude below that of $\mathrm{H}_{2}$ and $\mathrm{CO}$ over the whole temperature range at this density. In general, the relative cooling contributions of these molecules are expected to depend on the gas density, the amount of dust present and cosmic ray effects regulating the molecular chemistry.

The fine-structure line cooling produced by metal species is illustrated in Fig. 10. The top panel example shows the cooling function of oxygen expanded in its contributions from individual ionization stages OI-OVIII. The bottom panel of Fig. 10 gives the contributions from each metal element. In the lowtemperature regime the primary metal coolants are $\mathrm{C}, \mathrm{O}, \mathrm{Si}$, and $\mathrm{Fe}$. This is consistent with the findings of RSO14 for their radiation-free network. However, the importance of these metal elements for cooling at low temperature may be differently qualified when the effect of metal depletion onto dust grains is considered. As shown by Jenkins (2009) metal depletion can substantially diminish the fraction of metals in the gas phase, hence reducing their cooling contribution. The $\mathrm{NC}^{2} \mathrm{M}$ does not yet account for such depletion effects. In the high-temperature regime metal cooling is dominated by $\mathrm{C}$ and $\mathrm{O}$ between $2 \times$ $10^{4} \mathrm{~K} \lesssim T \lesssim 3.5 \times 10^{5} \mathrm{~K}$, and by Fe for $T \gtrsim 6 \times 10^{5} \mathrm{~K}$. Around $T \approx 5 \times 10^{5} \mathrm{~K}$ Ne provides an important contribution. The computation of metal line cooling was based on the recommendations summarized in Table 1 . When assuming only a five level atomic fine-structure model $(L=5)$ for each ion, the amount of cooling is underestimated by up to a factor of five, especially in the vicinity of $10^{6} \mathrm{~K}$ (dashed line in Fig. 10, bottom panel).

The total cooling function representing the sum of the distinctive cooling and heating agents is shown in Fig. 11. We recall that the following results are obtained for the full network at $n_{\mathrm{H}}=10^{6} \mathrm{~m}^{-3}$. There is net heating up to $50 \mathrm{~K}$ caused by the dominance of cosmic ray heating. There is a further narrow window of net heating between $T \approx 56 \mathrm{~K}$ and $T \approx 69 \mathrm{~K}$, which is the fingerprint of proton-collisional $\mathrm{H}_{2}$ excitations involving nuclear spin reversals (Eq. (58)). The corresponding cooling function for the radiation-free network in RSO14, Fig. 3, does not show this complicated structure below $100 \mathrm{~K}$ but states a unique thermal equilibrium temperature of about $100 \mathrm{~K}$. Ignoring Eq. (58) our model would likewise achieve a unique thermal equilibrium at $\sim 76 \mathrm{~K}$. The temperature regime from a few hundred Kelvin to $\sim 8000 \mathrm{~K}$ is governed by molecular cooling. The total cooling function reaches a maximum at $T \approx 1.6 \times 10^{4} \mathrm{~K}$, which is related to cooling processes of hydrogen and helium. Metal cooling then dominates over the large interval from $T \approx 3 \times 10^{4} \mathrm{~K}$ to $T \approx 10^{7} \mathrm{~K}$. Cooling due to Bremsstrahlung becomes unimportant before $\sim 10^{6} \mathrm{~K}$ but dominates above $\sim 10^{7} \mathrm{~K}$.

The impact of different densities, absence of dust, and absence of cosmic rays on the total cooling function is presented in Fig. 12. As can be seen the cooling behavior is insensitive to such influence in the high-temperature regime but reveals a strong dependence in the low-temperature regime. The absence of dust (but presence of cosmic rays) reduces molecule fractions and thus diminishes molecular cooling and therefore lowers total cooling (yellow curve in Fig. 12). In the absence of cosmic rays (but presence of dust) molecule fractions are higher and molecular cooling is increased, in particular below $T \approx 100 \mathrm{~K}$ (CO and $\left.\mathrm{H}_{2} \mathrm{O}\right)$ and above $T \approx 1000 \mathrm{~K}\left(\mathrm{H}_{2}\right)$. Because of this and because the low degree of ionization renders Eq. (58) unimportant, there is no net heating at $T \geq 10 \mathrm{~K}$ (light blue curve in Fig. 12). The cooling function for the full network at the lower density $n_{\mathrm{H}}=10^{4} \mathrm{~m}^{-3}$ is depicted as a red line in Fig. 12. Since cosmic ray heating is roughly proportional to the gas density it becomes relatively more important at lower densities than cooling processes, being proportional to the density squared in the low-density limit. Consequently, the thermal equilibrium temperature lies much higher at $T \approx 700 \mathrm{~K}$. For the full network with $n_{\mathrm{H}}=10^{10} \mathrm{~m}^{-3}$ there is no heating at $T \geq 10 \mathrm{~K}$, and the cooling efficiency is reduced by roughly one order of magnitude at $T \lesssim 1000 \mathrm{~K}$ (dark blue curve in Fig. 12) compared with the $n_{\mathrm{H}}=10^{6} \mathrm{~m}^{-3}$ case. The reduction comes about because, with increasing gas density, molecular cooling approaches the LTE regime, which lowers the cooling rate per $n_{\mathrm{H}_{\text {nuc }}}^{2}$, that is, $\Lambda_{*}$.

\section{Non-equilibrium chemistry and cooling}

The cooling functions computed for chemical equilibrium in the last section are now compared with cooling functions in timedependent situations where chemical equilibrium is not prevailing. To demonstrate the impact of non-equilibrium effects, the chemical rate equations and thermal rate equation are solved simultaneously under isochoric conditions using a modified version of the NIRVANA code operating on a single numerical cell. We focus on the isochoric case here since constant density is usually assumed during the chemistry and cooling update in a hydrodynamic simulation time step. Under isochoric assumption the temperature evolves according to

$\frac{\mathrm{d} T}{\mathrm{~d} t}=-\frac{(\gamma-1) \Lambda_{\text {net }}}{k n_{\mathrm{tot}}}-\frac{T}{n_{\mathrm{tot}}} \frac{\mathrm{d} n_{\mathrm{tot}}}{\mathrm{d} t}$ 

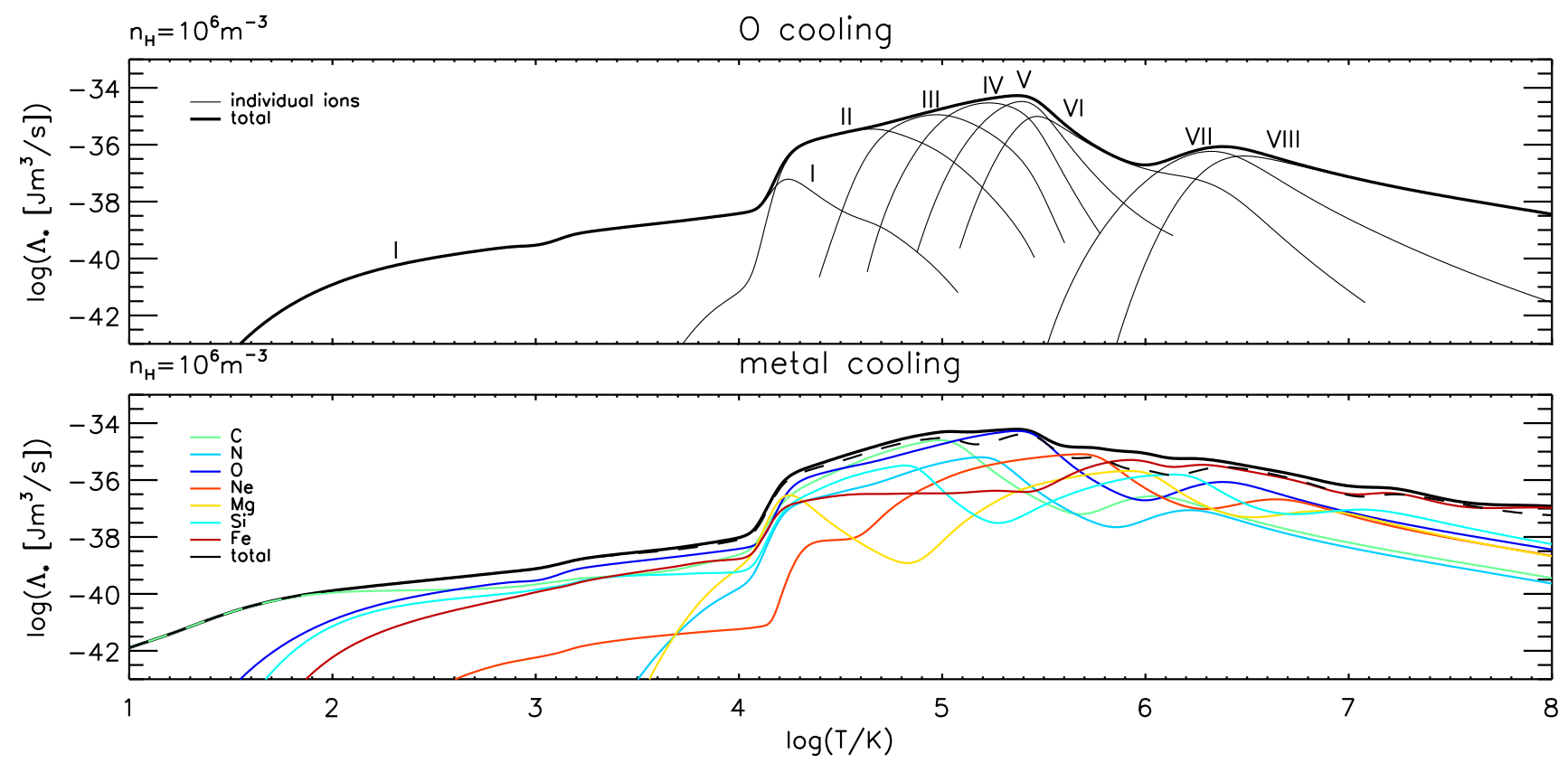

Fig. 10. Metal cooling in chemical equilibrium for the full network. Top panel: contribution of individual oxygen ionization stages. Bottom panel: contribution of different metals. The dashed line represents the total metal cooling when the number of energy levels is restricted to 5 ( $L=5$ ) in all ion computations.

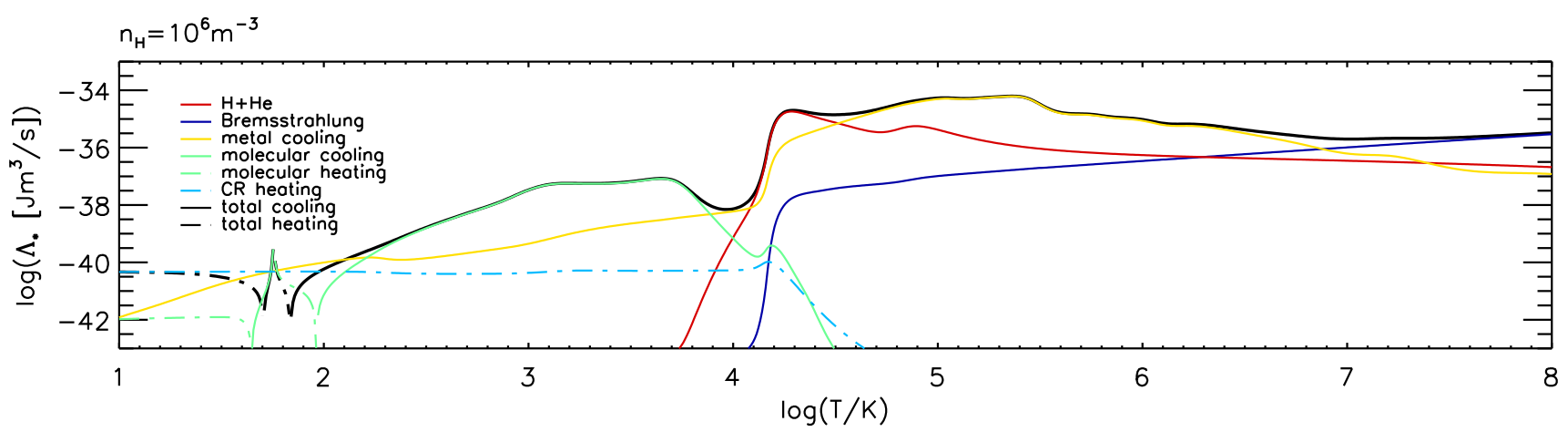

Fig. 11. Total cooling function and its contributions from different cooling or heating agents in chemical equilibrium for the full network. Dashdotted lines indicate heating.

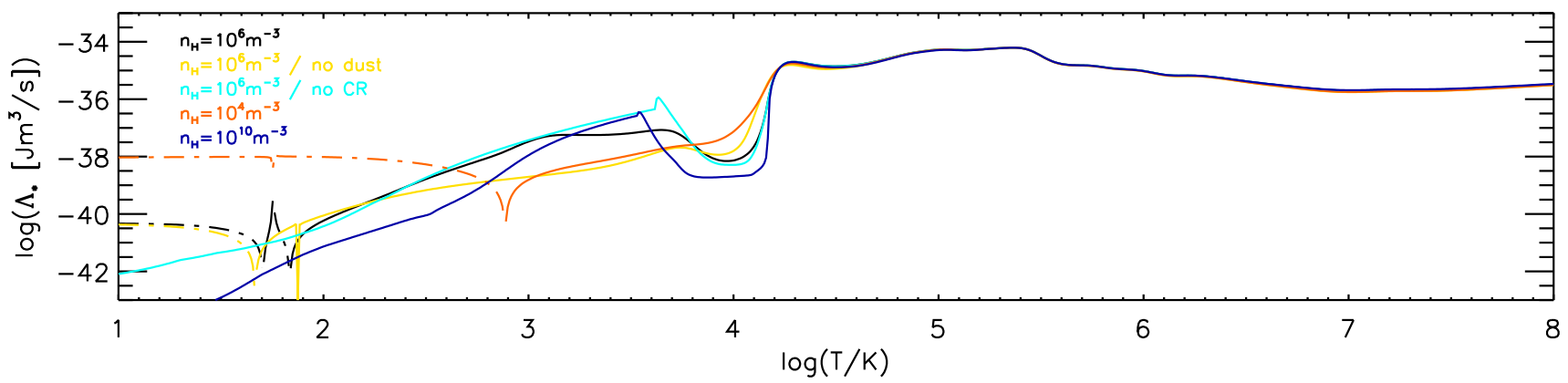

Fig. 12. Impact of different gas densities and the absence of dust or cosmic rays on the net cooling function in chemical equilibrium. Dash-dotted lines indicate net heating, $\Lambda_{*}<0$.

where the time derivative of total number density $n_{\text {tot }}$ can be replaced by making use of the reaction source terms as shown in Ziegler (2016). The adiabatic index is approximated by

$\gamma=\frac{5\left(n_{\mathrm{H}}+n_{\mathrm{He}}+n_{\mathrm{e}}\right)+7 n_{\mathrm{H}_{2}}}{3\left(n_{\mathrm{H}}+n_{\mathrm{He}}+n_{\mathrm{e}}\right)+5 n_{\mathrm{H}_{2}}}$ which approaches $5 / 3$ for atomic gas and $7 / 5$ for $\mathrm{H}_{2}$ molecular gas ${ }^{9}$ Integration of Eq. (87) allows us to represent chemical

9 Generally, the adiabatic index of $\mathrm{H}_{2}$ gas shows a dependence on temperature for $T \lesssim 400 \mathrm{~K}$ where $\gamma>7 / 5$, and the caloric equation of state is more complex in this temperature regime (see, e.g., Boley et al. 2007, Fig. 1). 


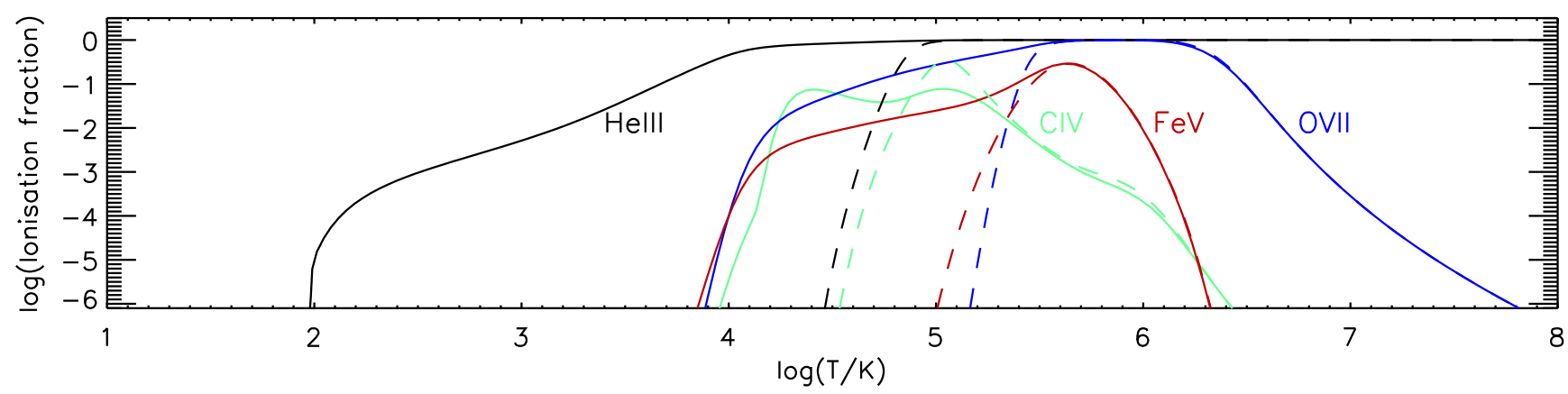

Fig. 13. Ionization fractions of HeIII, CIV, OVII, and FeV obtained for the full network in equilibrium (dashed lines) and non-equilibrium (solid lines) in the $10^{8} \mathrm{~K}$ cooling gas experiment.

abundances and cooling rates in non-equilibrium as a function of time-dependent temperature and enables us to compare those with ones obtained by assuming instantaneous chemical equilibrium.

Isochoric simulations of cooling gas are performed for two different densities. The first experiment with $n_{\mathrm{H}_{\text {nuc }}}=10^{6} \mathrm{~m}^{-3}$ starts from a temperature $T=10^{8} \mathrm{~K}$ and assumes that the gas is in chemical equilibrium initially. Over the course of evolution, cooling drives the gas towards lower temperatures up to the point where thermal equilibrium is reached and a new chemical equilibrium is established. Results of the computations for the full network and for the reduced networks (no dust and no cosmic rays) are displayed in the Figs. 13-15. Simulations have been tracked to the final equilibrium state with associated thermal equilibrium temperatures of $\sim 69 \mathrm{~K}$ and $\sim 74 \mathrm{~K}$ for the full network and dust-free network, respectively. The final equilibrium temperature for the CR-free network lies below $10 \mathrm{~K}$ outside of the presented temperature regime.

The most significant non-equilibrium effect is the development of ionization lags because the recombination of many ions drags behind cooling. It means that higher ionization stages of species exist down to lower temperatures compared to equilibrium. Such ionization lags are not an exclusive phenomenon of metal species but also appear for hydrogen and helium. This is illustrated in Fig. 13, which compares the computed ionization fractions for the selected ions HeIII, CIV, OVII, and $\mathrm{FeV}$ with their corresponding equilibrium fractions for the full network. All of the ions shown have comparatively long recombination times and therefore persist down to lower temperatures in non-equilibrium than predicted for equilibrium. In the case of HeIII, for instance, the ionization fraction does not fall below $1 \%$ before $\sim 1000 \mathrm{~K}$ whereas in equilibrium this temperature is $\sim 5 \times 10^{4} \mathrm{~K}$. The occurrence of the double peak for CIV at $T \approx 2.5 \times 10^{4} \mathrm{~K}$ and $T \approx 1.1 \times 10^{5} \mathrm{~K}$ is a further characteristic feature that occurs for metal ions where dielectronic recombination dominates radiative recombination in the temperature range between the two appearing maxima. In general, all the computed non-equilibrium ionization curves are qualitatively similar to those obtained in previous studies (e.g., Schmutzler \& Tscharnuter 1993; Gnat \& Sternberg 2007). The results were obtained for a fixed metallicity of $Z=0.96 Z_{\odot}$. For higher (lower) metallicities, cooling times are shorter (longer), and non-equilibrium ionization lags are expected to be greater (smaller), which has implications for the cooling efficiency (Oppenheimer \& Schaye 2013a).

The non-equilibrium cooling function is illustrated in Fig. 14. Results for the full network are discussed first, which are depicted as black lines in Fig. 14. Departures from the equilibrium become apparent for temperatures $T \lesssim 5 \times 10^{5} \mathrm{~K}$. This

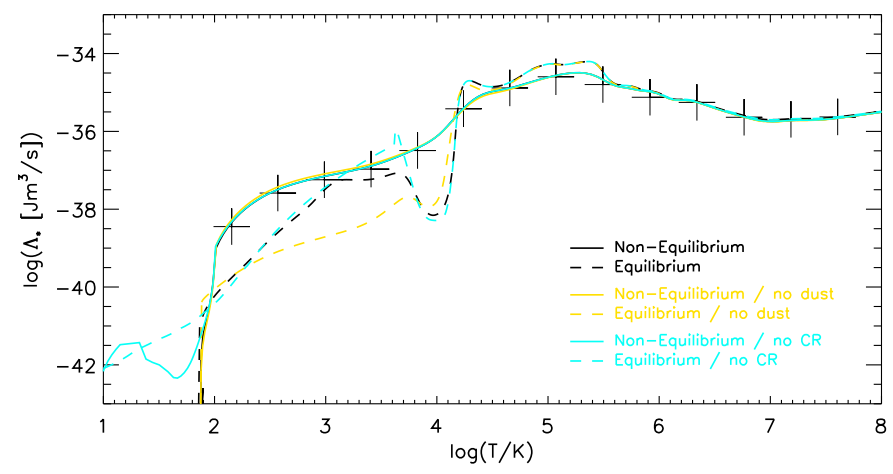

Fig. 14. Cooling curve in equilibrium (dashed lines) and in nonequilibrium (solid lines) for the full network (black), dust-free network (yellow), and CR-free network (blue). For $T \gtrsim 100 \mathrm{~K}$ the solid lines representing the different network cases nearly coincide. Crosses: Non-equilibrium cooling curve for a network without the metals $\mathrm{N}$, $\mathrm{Mg}$ and $\mathrm{Ne}$.

is the point when cooling proceeds more rapidly than recombination. At higher temperatures the cooling timescale is generally large so that the gas has sufficient time to approximately adjust chemical equilibrium yielding a close-to-equilibrium cooling rate. Down to $T \approx 1.6 \times 10^{4} \mathrm{~K}$ non-equilibrium cooling (solid line) is suppressed compared to equilibrium cooling (dashed line) by a maximum factor of three appearing near the hydrogen Ly $\alpha$ peak at about $2 \times 10^{4} \mathrm{~K}$. The distinct $\mathrm{H}$ Ly $\alpha$ bump and the unobtrusive bumps of the $\mathrm{C}, \mathrm{O}$, and $\mathrm{Ne}$ metal contributions present in the equilibrium cooling function are smeared out in non-equilibrium because of the larger overlap of generally broader ion distributions. The broadening of ion distributions due to ionization lags as seen in Fig. 13 leads to the observed suppression of cooling in this temperature regime. This is because the most effective ion coolants at a given temperature are less abundant than in ionization equilibrium.

On the other hand, non-equilibrium cooling is enhanced for $T \lesssim 1.6 \times 10^{4} \mathrm{~K}$. At $T \approx 10^{4} \mathrm{~K}$ the non-equilibrium rate is nearly two orders of magnitude higher than the equilibrium rate. In the equilibrium case metal cooling drops significantly around $1.6 \times 10^{4} \mathrm{~K}$ owing to a rapid decline of the density of free electrons before molecular cooling becomes important at even lower temperatures. In the non-equilibrium case, due to the recombination delay, a high electron number density exists down to $\sim 100 \mathrm{~K}$ as demonstrated in Fig. 15 (top panel). As a consequence metal cooling is enhanced by roughly two orders of magnitude compared with equilibrium. At the same time, molecular cooling is substantially diminished in non-equilibrium since the formation of $\mathrm{H}_{2}$ and other important molecular coolants takes too long and 

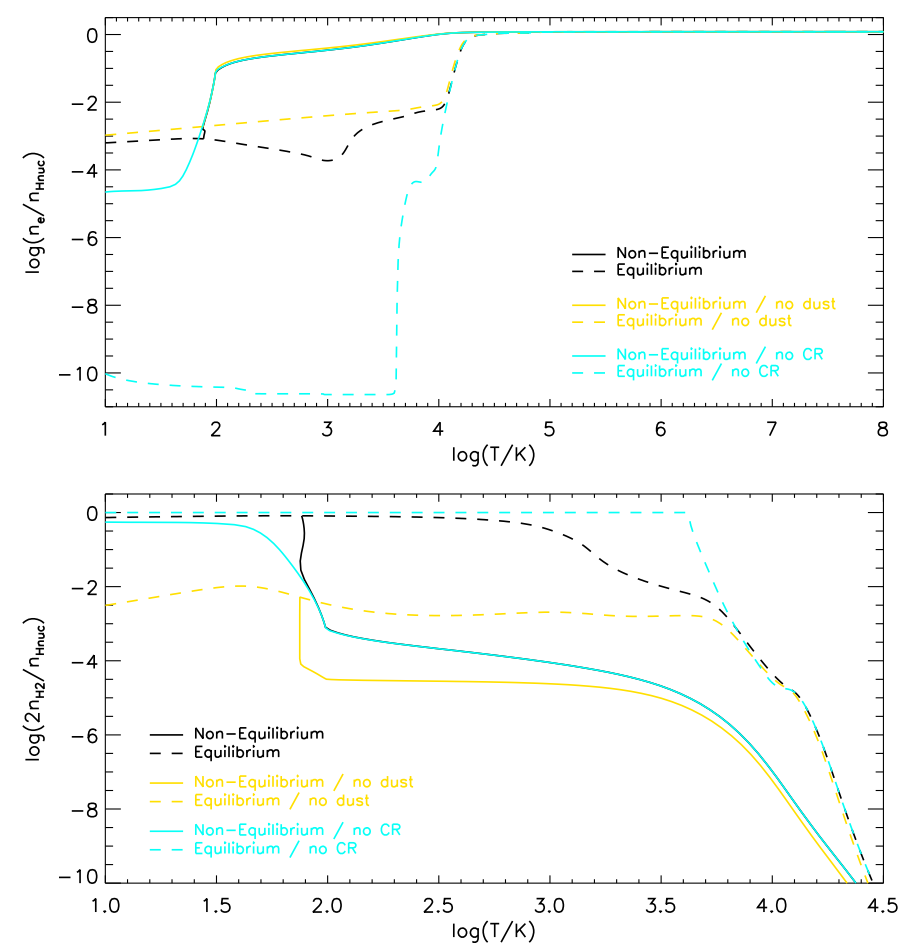

Fig. 15. Electron fraction (top panel) and molecular hydrogen fraction (bottom panel) as a function of temperature in equilibrium (dashed lines) and in non-equilibrium (solid lines) for the full network (black), dust-free network (yellow), and CR-free network (blue).

thus cannot keep up with cooling. The increase in metal cooling over-compensates the diminished molecular cooling resulting in an overall enhancement compared to equilibrium except in the close vicinity of $\sim 1000 \mathrm{~K}$ where $\mathrm{H}_{2} \mathrm{O}$ cooling peaks for the full network under equilibrium conditions (see Fig. 9).

The $T$-dependence of the $\mathrm{H}_{2}$ fraction in non-equilibrium is illustrated in Fig. 15 (bottom panel). At any temperature the non-equilibrium $\mathrm{H}_{2}$ fraction (solid line) remains below the equilibrium fraction (dashed line) with up to three orders of magnitude difference in the interval $100 \mathrm{~K}-1000 \mathrm{~K}$. At late times, when thermal equilibrium has been established, the $\mathrm{H}_{2}$ fraction takes on its equilibrium value at $T \approx 69 \mathrm{~K}$ (in Fig. 15 this is when the black solid line meets the dashed line). Just before equilibrium settles, the final equilibrium temperature is slightly undershot (in Fig. 15 this is when the curve has a vertical tangent for the first time) as a result of non-equilibrium cooling effects.

In Sect. 4.3 we observed that $\mathrm{N}$ and $\mathrm{Mg}$ give only minor contributions to the total metal cooling, and that Ne has some significant contribution only near $T \approx 5 \times 10^{5} \mathrm{~K}$. To study their neglect on the non-equilibrium cooling curve, the gas cooling experiment is repeated for a network without the elements $\mathrm{N}, \mathrm{Mg}$, and Ne. The result is represented by the crosses in Fig. 14. The deviations to the full network are largest near $T \approx 5 \times 10^{5} \mathrm{~K}$ due to missing Ne cooling but, in general, may be on an acceptable level. When including Ne the discrepancies become rather small, which means that $\mathrm{N}$ and $\mathrm{Mg}$ coolants may be safely ignored.

The non-equilibrium cooling curves obtained for the reduced networks are virtually indistinguishable from the respective cooling curve for the full network. Neither the absence of dust nor the absence of cosmic rays has a remarkable impact on the non-equilibrium cooling efficiency. This is because of the dominant role played by ionization lags and the relative unimportance of dust and cosmic rays in boostin molecule formation in cooling gas starting from a highly ionized state. The non-equilibrium
$\mathrm{H}_{2}$ fraction for both the dust-free network (yellow solid line in Fig. 15) and the CR-free network (blue solid line in Fig. 15) is some orders of magnitude below the corresponding equilibrium fraction (dashed lines in Fig. 15) down to $100 \mathrm{~K}$. Like for the full network, metal cooling is substantially enhanced in the temperature range $100 \mathrm{~K}-10^{4} \mathrm{~K}$ due to an increased number density of free electrons. In particular, the non-equilibrium electron fraction for the CR-free network (blue solid line in Fig. 15) is up to ten orders of magnitude larger compared to equilibrium (blue dashed line in Fig. 15). For example, at $T=1000 \mathrm{~K}$ the non-equilibrium value is $n_{\mathrm{e}} / n_{\mathrm{H}_{\text {nuc }}} \approx 0.3$ whereas in equilibrium, $n_{\mathrm{e}} / n_{\mathrm{H}_{\text {nuc }}} \approx 3 \times 10^{-11}$. The $T$-dependence of the nonequilibrium electron fraction is very alike for all three networks down to $100 \mathrm{~K}$ with slightly larger values for the dust-free network because of the missing ion neutralization on dust grains. Near $\sim 3000 \mathrm{~K}$ the non-equilibrium cooling function for the CRfree network falls below the equilibrium function, which is dominated near this temperature by relatively strong $\mathrm{H}_{2}$ cooling.

In a second experiment the cooling down of high-density gas with $n_{\mathrm{H}_{\text {nuc }}}=10^{10} \mathrm{~m}^{-3}$ and initial temperature of $T=10^{5} \mathrm{~K}$ was studied. Simulations were performed only for the full chemical network including dust and cosmic ray effects. Two different cases are considered that differ in their initial conditions. Case A starts from chemical equilibrium for $10^{5} \mathrm{~K}$. Case B starts with a chemical composition that corresponds to chemical equilibrium at a lower temperature of $100 \mathrm{~K}$. One may think here of a situation where gas is impulsively heated to a high temperature as it occurs in the interaction with high Mach number shocks. Because the gas in case B is predominantly in molecular form at the beginning, the initial phase of evolution is expected to differ significantly from case A. Both cases are contrasted with a solution where equilibrium abundances are imposed at all times. The simulations are stopped when the time-dependent temperature falls below $10 \mathrm{~K}$, which is larger than the thermal equilibrium value of $\sim 4.47 \mathrm{~K}$.

Figure 16 subsumes the results of these calculations displaying the time evolution of temperature (top panel) in units of days, and shows the net cooling rate (panel 2), electron fraction (panel 3 ), and $\mathrm{H}_{2}$ fraction (bottom panel) as a function of temperature for all cases. There is a one-to-one relation between time and temperature, which can be converted into each other. In case A the temperature starts to deviate significantly from the equilibrium solution after one hundred days. Subsequently, the nonequilibrium temperature (black solid line) remains substantially below the equilibrium temperature (dashed line) down to $\sim 80 \mathrm{~K}$, which is reached after $\sim 6 \times 10^{5} \mathrm{~d}$. For instance, when $T=100 \mathrm{~K}$ at $t \approx 6 \times 10^{4} \mathrm{~d}$, the equilibrium solution value at the same time is $T \approx 10^{4} \mathrm{~K}$. The different temperature histories are explained with the different cooling rates in the course of evolution. The cooling functions are illustrated in panel 2 of Fig. 16. As in the previous $10^{8} \mathrm{~K}$ experiment, the cooling in case $\mathrm{A}$ is affected by ionization lags, which increase the electron density at lower temperatures (see panel 3 of Fig. 16) and lead to stronger metal (and net) cooling between $T \approx 4500 \mathrm{~K}$ and $T \approx 1.6 \times 10^{4} \mathrm{~K}$ compared with equilibrium. Consequently, the temperature decreases more rapidly in this temperature regime. Figure 16 (panel 3) demonstrates that the electron fraction stays above $10^{-2}$ down to $T \approx 200 \mathrm{~K}$ whereas in the equilibium case it is of the order of $10^{-6}$ for $T \lesssim 10^{4} \mathrm{~K}$. The $\mathrm{H}_{2}$ fraction is diminished (Fig. 16, bottom panel), therefore the contribution of molecular cooling to the net cooling is reduced compared to equilibrium.

The results for case B are depicted as red lines in Fig. 16. Starting from $10^{5} \mathrm{~K}$ the temperature first falls below case A because the initially high $\mathrm{H}_{2}$ fraction provides more molecular 

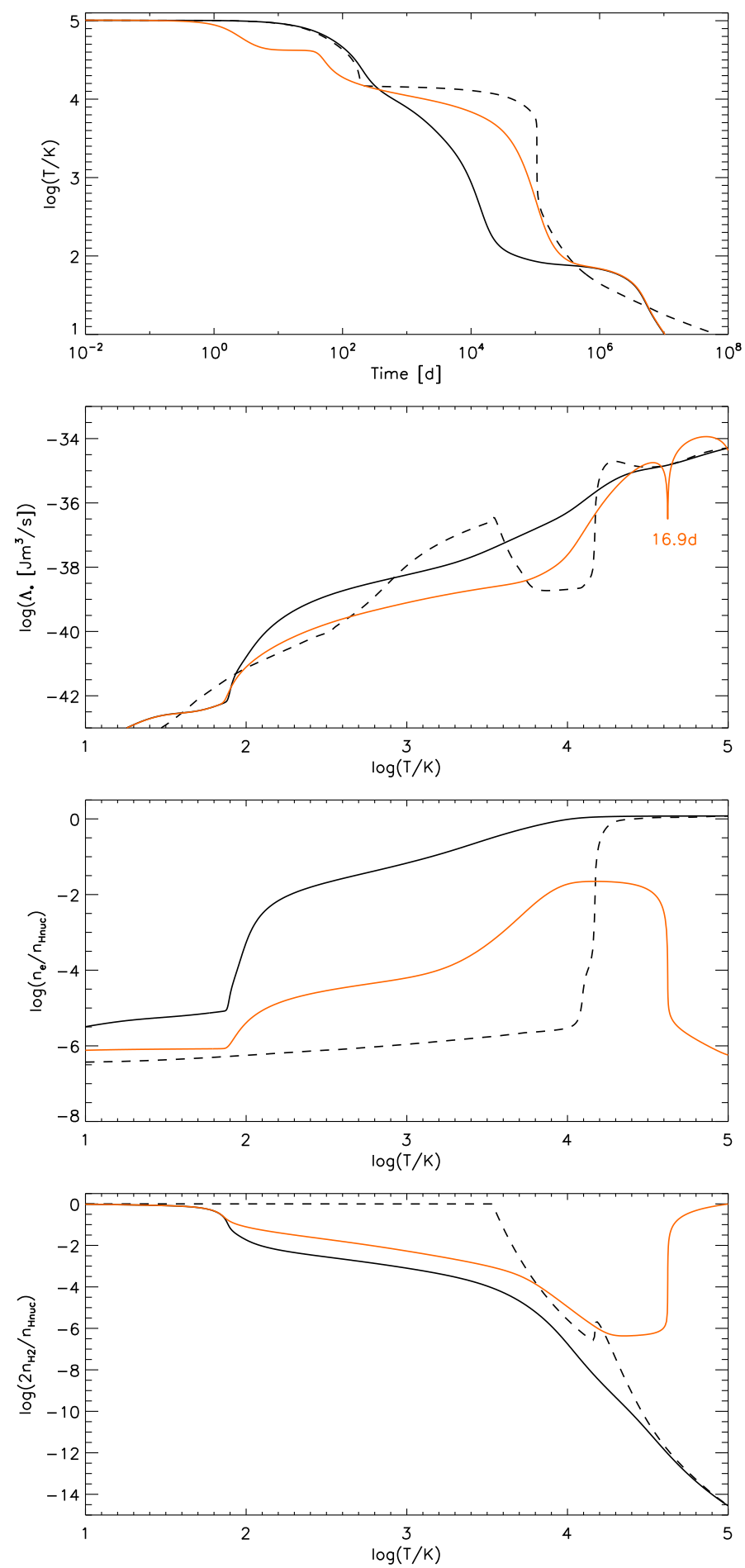

Fig. 16. Time dependence of the temperature (top panel) and the net cooling rate (panel 2), electron fraction (panel 3), and molecular hydrogen fraction (bottom panel) as a function of temperature for case A (black solid lines), case B (red lines), and for the equilibrium solution (dashed lines).

cooling and net cooling compared to both case A and the equilibrium case. This initial phase of enhanced net cooling is a rather short-term phenomenon and lasts for less than $\sim 16 \mathrm{~d}$ because rapid dissociation of molecules sets in. The dissociation boost halts at $t \approx 16.9 \mathrm{~d}\left(T \approx 4 \times 10^{4} \mathrm{~K}\right)$, which is striking in Fig. 16 as the sudden drop in the cooling rate and the sharp decline in the $\mathrm{H}_{2}$ fraction. The liberated atomic hydrogen is rapidly ionized and this produces a similarly sharp increase in the electron fraction. From $t \approx 300 \mathrm{~d}$ to $t \approx 6 \times 10^{5} \mathrm{~d}$ the temperature in case $\mathrm{B}$ exceeds the value for case $\mathrm{A}$ because of a lower net cooling rate between $T \approx 100 \mathrm{~K}$ and $T \approx 2 \times 10^{4} \mathrm{~K}$. The weaker cooling for case $\mathrm{B}$ in this temperature regime reflects the fact that ionization lags are less important, and atomic line cooling is closer to equilibrium. The $\mathrm{H}_{2}$ fraction is higher than in case $\mathrm{A}$ but remains relatively unimportant except in the very initial phase of evolution.

\section{Conclusions}

In this paper a chemistry and cooling module, $\mathrm{NC}^{2} \mathrm{M}$, for the multiphysics, adaptive-grid, and MPI-parallelized code NIRVANA has been presented. The $\mathrm{NC}^{2} \mathrm{M}$ has been checked successfully both by computing equilibrium solutions and performing time-dependent, non-equilibrium cooling gas simulations with an isochoric equation of state. NIRVANA now allows for more realistic simulations of interstellar gas by explicitly following non-equilibrium chemistry and thermal processes. The network of the $\mathrm{NC}^{2} \mathrm{M}$ includes chemical schemes for the important coolants $\mathrm{H}_{2}, \mathrm{CO}, \mathrm{H}_{2} \mathrm{O}$, and $\mathrm{OH}$ and covers a fully-fledged ionization model for the elements $\mathrm{H}, \mathrm{D}, \mathrm{He}, \mathrm{C}, \mathrm{N}, \mathrm{O}, \mathrm{Ne}, \mathrm{Mg}, \mathrm{Si}$, and $\mathrm{Fe}$. It accounts for dust catalysis $\left(\mathrm{H}_{2}\right.$ formation, ion neutralization) and treats cosmic ray effects (ionization, molecule dissociation). The implementation of cooling processes is state of the art. For example, atomic data and electron excitation coefficients for metal line cooling are based on the up-to-date Chianti database version 8 . The $\mathrm{NC}^{2} \mathrm{M}$ is seriously applicable within a wide range of temperatures and densities and can be used without restriction from a technical point of view. In extended mode it evolves 121 species linked by 426 chemical reactions. However, exploitation of the full power of the $\mathrm{NC}^{2} \mathrm{M}$ in multidimensional, gas-dynamical simulations is still extremely challenging in terms of computational costs. A reduction of complexity seems possible by neglecting the elements $\mathrm{N}, \mathrm{Mg}$, and Ne since $\mathrm{N}$ and $\mathrm{Mg}$ are minor coolants relative to other metals and $\mathrm{Ne}$ essentially contributes to cooling only near $T=5 \times 10^{5} \mathrm{~K}$.

Nevertheless, $\mathrm{NC}^{2} \mathrm{M}$ suffers from several weaknesses. The first important aspect concerns the opaqueness of the gas-dust mixture. At sufficiently high gas densities, atomic fine-structure lines and molecular rotovibrational lines become optically thick and are subject to dust absorption as well. In the $\mathrm{NC}^{2} \mathrm{M}$ atomic line cooling is treated in the optically thin approximation. Rotovibrational line cooling crudely respects the optically thick regime by either adopting a local-density-dependent attenuation factor in the case of $\mathrm{H}_{2}$ or by simply setting an upper threshold for the optical depth parameter in the cooling models for $\mathrm{CO}$ and $\mathrm{H}_{2} \mathrm{O}$. A more proper treatment of an optically thick, dusty medium would require the solution of a complex radiative transfer problem, which is currently beyond our capabilities. Second, in the present stage of development the $\mathrm{NC}^{2} \mathrm{M}$ ignores photoionization and photodissociation by incident radiation. The interstellar background radiation field as cataloged by Black (1987) possesses an ionizing UV component from starlight. In regions of unshielded gas, photochemical reactions can therefore have a strong impact on the ionization level and molecular chemistry in the low-temperature regime which, in turn, affects the cooling properties. Photoionization of hydrogen, helium, and metals cause a reduction of their cooling efficiencies because fewer bound electrons mean fewer line transitions. Third, dust in the $\mathrm{NC}^{2} \mathrm{M}$ is considered as a passive constituent. There is no timedependent treatment of dust destruction (thermal sputtering, sublimation) and dust formation processes (accretion, coagulation). 
Since $\mathrm{H}_{2}$ production and ion neutralization are potentially influenced by dust, such destruction and formation processes become important if their timescales are short compared to other relevant timescales. For instance, in the gas cooling simulations starting with a high temperature (Sect. 5), dust would be destroyed by thermal sputtering in a time shorter than the cooling time. At the same time, dust would not reform at an equal rate since dust growth by accretion is rather inefficient at high temperatures (Draine \& Salpeter 1979). Consequently, the influence of dust-catalytic reactions like $\mathrm{H}_{2}$ formation are likely to be overestimated in these experiments by assuming a fixed dust component. Future development efforts aim to remove at least some of these weaknesses.

Acknowledgements. I would like to thank the unknown referee for her or his dedicated report and many useful comments, which helped to improve the paper.

\section{References}

Abel, T., Bryan, G. L., \& Norman, M. L. 2002, Science, 295, 93 Adams, N. G., \& Smith, D. 1976a, Int. J. Mass Spectrom. Ion Phys., 21, 349 Adams, N. G., \& Smith, D. 1976b, J. Phys. B, 9, 1439

Adams, N. G., Smith, D., \& Grief, D. 1978, Int. J. Mass Spectrom. Ion Phys., 26, 405

Adams, N. G., Smith, D., \& Paulson, J. F. 1980, J. Chem. Phys., 72, 288

Adams, N. G., Smith, D., \& Millar, T. J. 1984, MNRAS, 211, 857

Aldrovandi, S. M. V., \& Pequignot, D. 1973, A\&A, 25, 137

Alge, E., Adams, N. G., \& Smith, D. 1983, J. Phys. B, 16, 1433

Andreazza, C. M., \& Singh, P. D. 1997, MNRAS, 287, 287

Anicich, V. G., Futrell, J. H., Huntress, W. T., et al. 1975, Int. J. Mass Spectrom. Ion Phys., 18, 63

Anicich, V. G., Huntress, W. T., \& Futrell, J. H. 1976, Chem. Phys. Lett., 40, 233 Arnaud, M., \& Raymond, J. 1992, ApJ, 398, 394

Azatyan, V. V., Aleksandrov, E. N., \& Troshin, A. F. 1975, Kinet. Cat., 16, 306 Badnell, N. R. 2006, ApJS, 167, 334

Barinovs, G., \& van Hemert, M. C. 2006, ApJ, 636, 923

Barlow, S. G. 1984, PhD Thesis, Univ. Colorado

Baulch, D. L., Cobos, C. J., Cox, R. A., et al. 1992, J. Phys. Chem. Ref. Data, 21,411

Black, J. H. 1981, MNRAS, 197, 553

Black, J. H. 1987, Interstellar Processes, (Berlin: Springer Verlag), Astrophysics and Space Science Library, 134, 731

Boley, A. C., Hartquist, T. W., Durisen, R. H., et al. 2007, ApJ, 660, L175

Borysow, A. 2002, A\&A, 390, 779

Borysow, A., Jorgensen, U. G., \& Fu, Y. 2001, JQSRT, 68, 235

Bray, I., Burgess, A., Fursa, D. V., et al. 2000, A\&AS, 146, 481

Bruhns, H., Kreckel, H., Miller, K. A., et al. 2010, Phys. Rev. A, 82, 042708

Burgess, A., \& Tully, J. A. 1992, A\&A, 254, 436

Cazaux, S., \& Tielens, A. G. G. M. 2002, ApJ, 575, L29

Cazaux, S., \& Tielens, A. G. G. M. 2004, ApJ, 604, 222

Cazaux, S., \& Spaans, M. 2004, ApJ, 611, 40

Cen, R. 1992, ApJS, 78, 341

Cohen, N., \& Westberg, K. R. 1979, J. Phys. Chem., 83, 46

Cohen, N., \& Westberg, K. R. 1983, J. Phys. Chem. Ref. Data, 12, 531

Cravens, T. E., \& Dalgarno, A. 1978, ApJ, 219, 750

Croft, H., Dickinson, A. S., \& Gadea, F. X. 1999, MNRAS, 304, 327

Dalgarno, A., \& Black, J. H. 1976, Rep. Progr. Phys., 39, 573

Dalgarno, A., \& Lepp, S. 1987, Astrochemistry (Dordrecht: Reidel)

Dalgarno, A., Du, M. L., \& You, J. H. 1990, ApJ, 349, 675

Dalgarno, A., Yan, M., \& Liu, W. 1999, ApJS, 125, 237

Dean, A. J., Davidson, D. F., \& Hanson, R. K. 1991, J. Phys. Chem., 95, 183

Del Zanna, G., Dere, K. P., Young, P. R., et al. 2015, A\&A, 582, A56

Donahue, M., \& Shull, J. M. 1991, ApJ, 383, 511

Dove, J. E., Rusk, A. C. M., Cribb, P. H., et al. 1987, ApJ, 318, 379

Dubernet, M. L., Gargaud, M., \& McCarroll, R. 1992, A\&A, 259, 373

Draine, B. T. 2002, in The Cold Universe: Saas-Fee Advanced Course, eds. D. Pfenniger, \& Y. Revaz (Springer Verlag), 32, 213

Draine, B. T., \& Salpeter, E. E. 1979, ApJ, 231, 77

Fairbairn, A. R. 1969, Proc. R. Soc. A, 312, 207

Faure, A., Gorfinkiel, J. D., \& Tennyson, J. 2004, MNRAS, 347, 323

Federer, W., Villinger, H., Howorka, F., et al. 1984, Phys. Rev. Lett., 52, 2084

Fehsenfeld, F. C. 1976, ApJ, 209, 638

Ferland, G. J., Peterson, B. M., Horne, K., et al. 1992, ApJ, 387, 95
Ferland, G. J., Porter, R. L., van Hoof, P. A. M., et al. 2013, Rev. Mex. Astron. Astrofis., 49, 137

Field, R. J., Körõs, E., Noyes, R. M. J., et al. 1972, J. Am. Soc., 94, 8649

Field, D., Adams, N. G., \& Smith, D. 1980, MNRAS, 192, 1

Flower, D. R., Le Bourlot, J., Pineau des Forêts, G., et al. 2000, MNRAS, 314, 753

Frank, P. 1986, Proc. Int. Symp. Rarefied Gas Dyn., 2, 422

Fryxell, B., Olson, K., Ricker, P., et al. 2000, ApJS, 131, 273

Furlanetto, S. R., \& Stoever, S. J. 2010, MNRAS, 404, 1869

Geppert, W. D., Thomas, R. D., Ehlerding, A., et al. 2005, J. Phys. Conf. Ser., 4, 26

Gerlich, D. 1982, in Symp. on Atomic and Surface Physics, eds. W. Lindinger,

F. Howorka, \& T. D. Märk (Dordrecht: Kluwer), 304

Gerlich, D., \& Horning, S. 1992, Chem. Rev., 92, 1509

Glover, S. C. O., \& Jappsen, A.-K. 2007, ApJ, 666, 1

Glover, S. C. O., \& Abel, T. 2008, MNRAS, 388, 1627

Glover, S. C. O., \& Clark, P. C. 2012, MNRAS, 421, 116

Glover, S. C. O., Federrath, C., Mac Low, M.-M., et al. 2010, MNRAS, 404, 2

Gnat, O., \& Sternberg, A. 2007, ApJS, 168, 213

Gnat, O., \& Ferland, G. J. 2012, ApJS, 199, 20

Grassi, T., Bovino, S., Schleicher, D. R. G., et al. 2014, MNRAS, 439, 2386

Gray, C. 2002, Rose-Hulman Undergraduate Math. J., 3

Gray, W. J., Scannapieco, E., \& Kasen, D. 2015, ApJ, 801, 107

Gredel, R., Lepp, S., \& Dalgarno, A. 1987, ApJ, 323, L137

Gredel, R., Lepp, S., Dalgarno, A., et al. 1989, ApJ, 347, 289

Grevesse, N., \& Sauval, A. J. 1998, Space Sci. Rev., 85, 161

Guberman, S. L. 1995, J. Phys. Chem., 102, 1699

Gustafsson, M., \& Frommhold, L. 2003, A\&A, 400, 1161

Harding, L. B., Guadagnini, R., \& Schatz, G. C. 1993, J. Phys. Chem., 97, 5472

Herbst, E., \& Klemperer, W. 1973, ApJ, 185, 505

Hirano, S., \& Yoshida, N. 2013, ApJ, 763, 52

Hollenbach, D., \& McKee, C. F. 1979, ApJS, 41, 555

Hummer, D. G., \& Storey, P. J. 1998, MNRAS, 297, 1073

Huq, M. S., Doverspike, L. D., Champion, R. L., et al. 1982, J. Phys. B, 15, 951 Indriolo, N., \& McCall, B. J. 2012, ApJ, 745, 91

Janev, R. K., Langer, W. D., Evans, K. J., et al. 1987, Elementary Processes in Hydrogen-Helium Plasmas (Berlin: Springer Verlag)

Jenkins, E. B. 2009, ApJ, 700, 1299

Jensen, M. J., Bilodeau, R. C., Safvan, C. P., et al. 2000, ApJ, 543, 764

Jones, J. D. C., Birkinshaw, K., \& Twiddy, N. D. 1981, Chem. Phys. Lett., 77, 484

Jorgensen, U. G., Hammer, D., Borysow, A., et al. 2000, A\&A, 361, 283

Karpas, Z., Anicich, V., \& Huntress, W. T. 1979, J. Chem. Phys., 70, 2877

Kim, J. K., Theard, L. P., \& Huntress, W. T. 1974, Int. J. Mass Spectrom. Ion Phys., 15, 223

Kimura, M., Lane, N. F., Dalgarno, A., et al. 1993, ApJ, 405, 801

Kingdon, J. B., \& Ferland, G. J. 1996, ApJS, 106, 205

Klippenstein, S. J., \& Georgievskii, Y. 2010, J. Phys. Chem. A, 114, 278

Krumholz, M. R., Leroy, A. K., \& McKee, C. F. 2011, ApJ, 731, 25

Langer, W. D. 1978, ApJ, 225, 860

Lotz, W. 1967, ApJS, 14, 207

Larson, Å., Le Padellec, A., Semaniak, J., et al. 1998, ApJ, 505, 459

Lesaffre, P., Chieze, J.-P., Cabrit, S., et al. 2004, A\&A, 427, 147

Lesaffre, P., Gerin, M., Hennebelle, P., et al. 2007, A\&A, 469, 949

Le Petit, F., Nehmé, C., Le Bourlot, J., \& Roueff, E. 2006, ApJS, 164, 506

Le Teuff, Y. H., Millar, T. J., \& Marwick, A. J. 2000, A\&AS, 146, 157

Lepp, S., \& Shull, J. M. 1983, ApJ, 270, 578

Lim, A. J., Rawlings, J. M. C., \& Williams, D. A. 1999, MNRAS, 308, 1126

Linder, F., Janev, R. K., \& Botero, J. 1995, Atomic and Molecular Processes in Fusion Edge Plasmas (New York: Plenum Press), 397

Lipovka, A., Núñez-López, R., \& Avila-Reese, V. 2005, MNRAS, 361, 850

MacGregor, M., \& Berry, R. S. 1973, J. Phys. B, 6, 181

Maloney, P. R., Hollenbach, D. J., \& Tielens, A. G. G. M. 1996, ApJ, 466, 561

Massaglia, S., Mignone, A., \& Bodo, G. 2005, A\&A, 442, 549

Martin, P. G., Keogh, W. J., \& Mandy, M. E. 1998, ApJ, 499, 793

Martinez, Jr., O., Betts, N. B., Villano, S. M., et al. 2008, ApJ, 686, 1486

Mauclaire, G., Derai, R., \& Marx, R. 1978a, Int. J. Mass Spectrom. Ion Phys., 26, 284

Mauclaire, G., Derai, R., \& Marx, R. 1978b, Dyn. Mass Spectrom., 5, 139

McElroy, D., Walsh, C., Markwick, A. J., et al. 2013, A\&A, 550, A36

MacLow, M. M., \& Shull, J. M. 1986, ApJ, 302, 585

McEwan, M. J., Scott, G. B. I., Adams, N. G., et al. 1999, ApJ, 513, 287

Meijerink, R., \& Spaans, M. 2005, A\&A, 436, 397

Mielke, S. L., Perterson, K. A., Schwenke, D. W., et al. 2003, Phys. Rev. Lett., 91, 063201

Milligan, D. B., \& McEwan, M. J. 2000, Chem. Phys. Lett., 319, 482

Mitchell, G. F. 1984, ApJS, 54, 81

Mitchell, J. B. A. 1990, Phys. Rep., 186, 215 
Mitchell, G. F., \& Deveau, T. J. 1983, ApJ, 266, 646

Murrell, J. N., \& Rodriguez, J. A. 1986, J. Mol. Struct. Theochem., 139, 267

Natarajan, K., \& Roth, P. 1987, Combust. Flame, 70, 267

Nelson, R. P., \& Langer, W. D. 1999, ApJ, 524, 923

Neufeld, D. A., \& Kaufman, M. J. 1993, ApJ, 418, 263

Neufeld, D. A., Lepp, S., \& Melnick, G. J. 1995, ApJS, 100, 132

Novotny, O., Berg, M. H., Buhr, H., et al. 2010, J. Phys. Chem. A, 114, 4870

Oldenborg, R. C., Loge, G. W., Harradine, D. M., et al. 1992, J. Phys. Chem. 96,8426

Omukai, K. 2000, ApJ, 534, 809

Omukai, K., Tsuribe, T., Schneider, R., et al. 2005, ApJ, 626, 627

Oppenheimer, B. D., \& Schaye, J. 2013a, MNRAS, 434, 1043

Oppenheimer, B. D., \& Schaye, J. 2013b, MNRAS, 434, 1063

Orel, A. E. 1987, J. Chem. Phys., 87, 314

Osterbrock, D. E., \& Ferland, G. J. 2005, Astrophysics of Gaseous Nebulae and Active Galactic Nuclei, (University Science Books)

Petuchowski, S. J., Dwek, E., Allen, Jr., J. E., et al. 1989, ApJ, 342, 406

Poulaert, G., Brouillard, F., Claeys, W., et al. 1978, J. Phys. B, 11, L671

Prasad, S. S., \& Huntress, W. T. 1980, ApJS, 43, 1

Raksit, A. B., \& Warneck, P. 1980, J. Chem. Soc. Faraday Trans., 76, 1084

Ramaker, D. E., \& Peek, J. M. 1976, Phys. Rev. A, 13, 58

Richings, A. J., Schaye, J., \& Oppenheimer, B. D. 2014, MNRAS, 440, 3349

Ripamonti, E., \& Abel, T. 2004, MNRAS, 348, 1019

Rodgers, S. D., \& Millar, T. J. 1996, MNRAS, 280, 1046

Rosén, S., Peverall, R., Larsson, M., et al. 1998, Phys. Rev., 57, 4462

Rosén, S., Derkatch, A., Semaniak, J., et al. 2000, Faraday Discuss., 115, 295

Shavitt, I. 1959, J. Chem. Phys., 31, 1359

Savin, D. W. 2002, ApJ, 566, 599

Savin, D. W., Krstic, P. S., Haiman, Z., et al. 2004, ApJ, 606, L167

Schmutzler, T., \& Tscharnuter, W. M. 1993, A\&A, 273, 318

Schneider, I. F., Dulieu, O., Giusti-Suzor, A., et al. 1994, ApJ, 424, 983

Schulz, G. J., \& Asundi, R. K. 1967, Phys. Rev., 158, 25

Scott, G. B. I., Fairley, D. A., Freeman, C. G., et al. 1997, J. Chem. Phys., 106, 3982

Semenov, D., Hersant, F., Wakelam, V., et al. 2010, A\&A, 522, A42

Shapiro, P. R., \& Kang, H. 1987, ApJ, 318, 32

Shull, J. M., \& van Steenberg, M. 1982, ApJS, 48, 95

Sidhu, K. S., Miller, S., \& Tennyson, J. 1992, A\&A, 255, 453

Silk, J. 1970, Astrophys. Lett., 5, 283

Singh, P. D., Sanzovo, G. C., Borin, A. C., et al. 1999, MNRAS, 303, 235

Smith, D., Spanel, P., \& Mayhew, C. A. 1992, Int. J. Mass spectrom. Ion Proc., 117,457
Smith, D., \& Adams, N. G. 1977a, Int. J. Mass Spectrom. Ion Phys., 23, 123 Smith, D., \& Adams, N. G. 1977b, Chem. Phys. Lett., 47, 383

Smith, D., Adams, N. G., \& Miller, T. M. 1978, J. Chem. Phys., 69, 308 Smith, M. A., Schlemmer, S., von Richthofen, J., et al. 2002, ApJ, 578, L87 Smith, I. W. M., Herbst, E., \& Chang, Q. 2004, MNRAS, 350, 323

Spitzer, Jr., L. 1978, Physical Processes in the Interstellar Medium (New York: Wiley)

Stancil, P. C., \& Dalgarno, A. 1998, Faraday Discuss., 109, 61

Stancil, P. C., Schultz, D. R., Raković, M., et al. 2015, Charge Transfer Database http: //www . physast.uga. edu/ugacxdb/

Stone, J. M., \& Norman, M. L. 1992, ApJS, 80, 791

Sutherland, R. S., \& Dopita, M. A. 1993, ApJS, 88, 253

Takagi, H., Kosugi, N., \& Le Dourneuf, M. 1991, J. Phys. B, 24, 711

Teşileanu, O., Mignone, A., \& Massaglia, S. 2008, A\&A, 488, 429

Teyssier, R. 2002, A\&A, 385, 337

Trevisan, C. S., \& Tennyson, J. 2002, Plasma Phys. Control. Fusion, 44, 1263

Tsang, W., \& Hampson, R. F. 1986, J. Phys. Chem. Ref. Data, 15, 1087

Vasiliev, E. O. 2013, MNRAS, 431, 638

Verner, D. A., \& Ferland, G. J. 1996, ApJS, 103, 467

Viggiano, A. A., Howorka, F., Albritton, D. L., et al. 1980, ApJ, 236, 492

Voronov, G. S. 1997, At. Data Nucl. Data Tables, 65, 1

Wagner-Redeker, W., Kemper, P. R., Jarrold, M. F., et al. 1985, J. Chem. Phys., 83,1121

Walkauskas, L. P., \& Kaufman, F. 1975, Symp. Int. Combust. Proc., 15, 691

Wakelam, V., Selsis, F., Herbst, E., et al. 2005, A\&A, 444, 883

Wakelam, V., Herbst, E., Loison, J.-C., et al. 2012, ApJS, 199, 21

Warnatz, J. 1984, in Combustion Chemistry, ed. W. C. Gardiner, Jr. (NY: Springer-Verlag), 197

Watson, W. D., Anicich, V. G., \& Huntress, W. T. 1976, ApJ, 205, L165

Weingartner, J. C., \& Draine, B. T. 2001a, ApJ, 548, 296

Weingartner, J. C., \& Draine, B. T. 2001b, ApJ, 563, 842

Weingartner, J. C., \& Draine, B. T. 2001c, ApJS, 134, 263

Williams, J. P., Bergin, E. A., \& Caselli, P. 1998, ApJ, 503, 689

Wishart, A. W. 1979, MNRAS, 187, 59P

Woodall, J., Agúndez, M., Markwick-Kemper, A. L., et al. 2007, A\&A, 466, 1197

Yan, M. 1997, PhD Thesis, Center for Astrophysics, Harvard University

Ziegler, U. 2008, CPC, 179, 227

Ziegler, U. 2011, JCP, 230, 1035

Ziegler, U. 2012, SIAM J. Sci. Comput., 34, C102

Ziegler, U. 2016, A\&A, 586, A82

Zygelman, B., Dalgarno, A., Kimura, M., et al. 1989, Phys. Rev. A, 40, 2340 


\section{Appendix A: The chemical network}

Table A.1. $\mathrm{NC}^{2} \mathrm{M}$ - gas-phase reactions.

\begin{tabular}{llcl}
\hline \hline No. & Reaction & Notes & Ref. \\
\hline 1 & $\mathrm{H}+\mathrm{e}^{-} \rightarrow \mathrm{H}^{+}+2 \mathrm{e}^{-}$ & & 1 \\
2 & $\mathrm{H}^{+}+\mathrm{e}^{-} \rightarrow \mathrm{H}$ & Case A & 2 \\
3 & $\mathrm{He}^{-} \mathrm{e}^{-} \rightarrow \mathrm{He}^{+}+2 \mathrm{e}^{-}$ & & 1 \\
$4^{a}$ & $\mathrm{He}^{+}+\mathrm{e}^{-} \rightarrow \mathrm{He}$ & Case A & 3,4 \\
5 & $\mathrm{He}^{+}+\mathrm{e}^{-} \rightarrow \mathrm{He}^{2+}+2 \mathrm{e}^{-}$ & & 1 \\
6 & $\mathrm{He}^{2+}+\mathrm{e}^{-} \rightarrow \mathrm{He}^{+}$ & Case A & 2 \\
7 & $\mathrm{He}^{+}+\mathrm{H} \rightarrow \mathrm{He}^{+} \mathrm{H}^{+}$ & & 5 \\
8 & $\mathrm{He}^{+} \mathrm{H}^{+} \rightarrow \mathrm{He}^{+}+\mathrm{H}$ & & 6 \\
$9-14$ & $\mathrm{C}^{n+}+\mathrm{e}^{-} \rightarrow \mathrm{C}^{(n+1)+}+2 \mathrm{e}^{-}$ & $n=0 \ldots 5$ & 7 \\
\hline
\end{tabular}

Notes. ${ }^{(a)}$ Both RR and DR.

References. 1: Janev et al. (1987), 2: Ferland et al. (1992), 3: Hummer \& Storey (1998), 4: Aldrovandi \& Pequignot (1973), 5: Zygelman et al. (1989), 6: Kimura et al. (1993), 7: Voronov (1997), 8: Badnell (2006), 9: Stancil et al. (2015), 10: Shull \& van Steenberg (1982), 11: Arnaud \& Raymond (1992), 12: Wishart (1979), 13: Bruhns et al. (2010), 14: Ramaker \& Peek (1976), 15: Karpas et al. (1979), 16: Savin et al. (2004), 17: Trevisan \& Tennyson (2002), 18: MacLow \& Shull (1986), 19: Lepp \& Shull (1983), 20: Croft et al. (1999), 21: Poulaert et al. (1978), 22: Schneider et al. (1994), 23: Dalgarno \& Lepp (1987), 24: Orel (1987), 25: Abel et al. (2002), 26: Cohen \& Westberg (1983), 27: Martin et al. (1998), 28: Shapiro \& Kang (1987), 29: McElroy et al. (2013), 30: Walkauskas \& Kaufman (1975), 31: Dove et al. (1987), 32: Barlow (1984), 33: Gerlich \& Horning (1992), 34: Woodall et al. (2007), 35: Linder et al. (1995), 36: Sidhu et al. (1992), 37: Schulz \& Asundi (1967), 38: Huq et al. (1982), 39: Savin (2002), 40: Gerlich (1982), 41: Mielke et al. (2003), 42: Glover \& Abel (2008), 43: Shavitt (1959), 44: Stancil \& Dalgarno (1998), 45: Prasad \& Huntress (1980), 46: Dean et al. (1991), 47: Andreazza \& Singh (1997), 48: Dalgarno et al. (1990), 49: Singh et al. (1999), 50: Barinovs \& van Hemert (2006), 51: Adams et al. (1984), 52: Natarajan \& Roth (1987), 53: Milligan \& McEwan (2000), 54: Smith et al. (1978), 55: Adams et al. (1980), 56: Mitchell \& Deveau (1983), 57: Fairbairn (1969), 58: Smith et al. (1992), 59: Adams \& Smith (1976a), 60: Adams \& Smith (1976b), 61: Azatyan et al. (1975), 62: Smith et al. (2004), 63: Smith \& Adams (1977a), 64: Smith \& Adams (1977b), 65: Alge et al. (1983), 66: Harding et al. (1993), 67: Baulch et al. (1992), 68: Murrell \& Rodriguez (1986), 69: MacGregor \& Berry (1973), 70: Takagi et al. (1991), 71: McEwan et al. (1999), 72: Viggiano et al. (1980), 73: Frank (1986), 74: Larson et al. (1998), 75: Mitchell (1990), 76: Fehsenfeld (1976), 77: Field et al. (1980), 78: Tsang \& Hampson (1986), 79: Oldenborg et al. (1992), 80: Dubernet et al. (1992), 81: Guberman (1995), 82: Jones et al. (1981), 83: Cohen \& Westberg (1979), 84: Mauclaire et al. (1978a), 85: Mauclaire et al. (1978b), 86: Kim et al. (1974), 87: Anicich et al. (1975), 88: Anicich et al. (1976), 89: Watson et al. (1976), 90: Martinez et al. (2008), 91: Rosén et al. (2000), 92: Raksit \& Warneck (1980), 93: Novotny et al. (2010), 94: Jensen et al. (2000), 95: Mitchell (1984), 96: Klippenstein \& Georgievskii (2010), 97: Petuchowski et al. (1989), 98: Rosén et al. (1998), 99: Federer et al. (1984), 100: Scott et al. (1997), 101: Geppert et al. (2005), 102: Adams et al. (1978), 103: Smith et al. (2002), 104: Wagner-Redeker et al. (1985), 105: Glover et al. (2010) and 106: Warnatz (1984).
Table A.1. continued.

\begin{tabular}{|c|c|c|c|}
\hline No. & Reaction & Notes & Ref. \\
\hline $15-20^{a}$ & $\mathrm{C}^{(n+1)+}+\mathrm{e}^{-} \rightarrow \mathrm{C}^{n+}$ & $n=0 \ldots 5$ & 8 \\
\hline $21-24$ & $\mathrm{C}^{(n+1)+}+\mathrm{H} \rightarrow \mathrm{C}^{n+}+\mathrm{H}^{+}$ & $n=0 \ldots 3$ & 9 \\
\hline 25 & $\mathrm{C}+\mathrm{H}^{+} \rightarrow \mathrm{C}^{+}+\mathrm{H}$ & & 9 \\
\hline 26,27 & $\mathrm{C}^{(n+1)+}+\mathrm{He} \rightarrow \mathrm{C}^{n+}+\mathrm{He}^{+}$ & $n=2,3$ & 9 \\
\hline $28-34$ & $\mathrm{~N}^{n+}+\mathrm{e}^{-} \rightarrow \mathrm{N}^{(n+1)+}+2 \mathrm{e}^{-}$ & $n=0 \ldots 6$ & 7 \\
\hline $35-41^{a}$ & $\mathrm{~N}^{(n+1)+}+\mathrm{e}^{-} \rightarrow \mathrm{N}^{n+}$ & $n=0 \ldots 6$ & 8 \\
\hline $42-45$ & $\mathrm{~N}^{(n+1)+}+\mathrm{H} \rightarrow \mathrm{N}^{n+}+\mathrm{H}^{+}$ & $n=0 \ldots 3$ & 9 \\
\hline 46 & $\mathrm{~N}+\mathrm{H}^{+} \rightarrow \mathrm{N}^{+}+\mathrm{H}$ & & 9 \\
\hline $47-49$ & $\mathrm{~N}^{(n+1)+}+\mathrm{He} \rightarrow \mathrm{N}^{n+}+\mathrm{He}^{+}$ & $n=1 \ldots 3$ & 9 \\
\hline $50-57$ & $\mathrm{O}^{n+}+\mathrm{e}^{-} \rightarrow \mathrm{O}^{(n+1)+}+2 \mathrm{e}^{-}$ & $n=0 \ldots 7$ & 7 \\
\hline $58-65^{a}$ & $\mathrm{O}^{(n+1)+}+\mathrm{e}^{-} \rightarrow \mathrm{O}^{n+}$ & $n=0 \ldots 7$ & 8 \\
\hline $66-69$ & $\mathrm{O}^{(n+1)+}+\mathrm{H} \rightarrow \mathrm{O}^{n+}+\mathrm{H}^{+}$ & $n=0 \ldots 3$ & 9 \\
\hline 70 & $\mathrm{O}+\mathrm{H}^{+} \rightarrow \mathrm{O}^{+}+\mathrm{H}$ & & 9 \\
\hline $71-73$ & $\mathrm{O}^{(n+1)+}+\mathrm{He} \rightarrow \mathrm{O}^{n+}+\mathrm{He}^{+}$ & $n=1 \ldots 3$ & 9 \\
\hline $74-83$ & $\mathrm{Ne}^{n+}+\mathrm{e}^{-} \rightarrow \mathrm{Ne}^{(n+1)+}+2 \mathrm{e}^{-}$ & $n=0 \ldots 9$ & 7 \\
\hline $84-93^{a}$ & $\mathrm{Ne}^{(n+1)+}+\mathrm{e}^{-} \rightarrow \mathrm{Ne}^{n+}$ & $n=0 \ldots 9$ & 8 \\
\hline 94-96 & $\mathrm{Ne}^{(n+1)+}+\mathrm{H} \rightarrow \mathrm{Ne}^{n+}+\mathrm{H}^{+}$ & $n=1 \ldots 3$ & 9 \\
\hline 97-99 & $\mathrm{Ne}^{(n+1)+}+\mathrm{He} \rightarrow \mathrm{Ne}^{n+}+\mathrm{He}^{+}$ & $n=1 \ldots 3$ & 9 \\
\hline $100-111$ & $\mathrm{Mg}^{n+}+\mathrm{e}^{-} \rightarrow \mathrm{Mg}^{(n+1)+}+2 \mathrm{e}^{-}$ & $n=0 \ldots 11$ & 7 \\
\hline $112-123^{a}$ & $\mathrm{Mg}^{(n+1)+}+\mathrm{e}^{-} \rightarrow \mathrm{Mg}^{n+}$ & $n=0 \ldots 11$ & 8 \\
\hline $124-126$ & $\mathrm{Mg}^{(n+1)+}+\mathrm{H} \rightarrow \mathrm{Mg}^{n+}+\mathrm{H}^{+}$ & $n=1 \ldots 3$ & 9 \\
\hline 127,128 & $\mathrm{Mg}^{n+}+\mathrm{H}^{+} \rightarrow \mathrm{Mg}^{(n+1)+}+\mathrm{H}$ & $n=0,1$ & 9 \\
\hline 129,130 & $\mathrm{Mg}^{(n+1)+}+\mathrm{He} \rightarrow \mathrm{Mg}^{n+}+\mathrm{He}^{+}$ & $n=2,3$ & 9 \\
\hline 131-144 & $\mathrm{Si}^{n+}+\mathrm{e}^{-} \rightarrow \mathrm{Si}^{(n+1)+}+2 \mathrm{e}^{-}$ & $n=0 \ldots 13$ & 7 \\
\hline $145-158^{a}$ & $\mathrm{Si}^{(n+1)+}+\mathrm{e}^{-} \rightarrow \mathrm{Si}^{n+}$ & $n=0 \ldots 13$ & 8 \\
\hline $159-161$ & $\mathrm{Si}^{(n+1)+}+\mathrm{H} \rightarrow \mathrm{Si}^{n+}+\mathrm{H}^{+}$ & $n=1 \ldots 3$ & 9 \\
\hline 162,163 & $\mathrm{Si}^{n+}+\mathrm{H}^{+} \rightarrow \mathrm{Si}^{(n+1)+}+\mathrm{H}$ & $n=0,1$ & 9 \\
\hline 164,165 & $\mathrm{Si}^{(n+1)+}+\mathrm{He} \rightarrow \mathrm{Si}^{n+}+\mathrm{He}^{+}$ & $n=2,3$ & 9 \\
\hline 166 & $\mathrm{Si}+\mathrm{He}^{+} \rightarrow \mathrm{Si}^{+}+\mathrm{He}$ & & 9 \\
\hline 167-192 & $\mathrm{Fe}^{n+}+\mathrm{e}^{-} \rightarrow \mathrm{Fe}^{(n+1)+}+2 \mathrm{e}^{-}$ & $n=0 \ldots 25$ & 7 \\
\hline $193-218^{a}$ & $\mathrm{Fe}^{(n+1)+}+\mathrm{e}^{-} \rightarrow \mathrm{Fe}^{n+}$ & $n=0 \ldots 25$ & $8,10,11$ \\
\hline 219-221 & $\mathrm{Fe}^{(n+1)+}+\mathrm{H} \rightarrow \mathrm{Fe}^{n+}+\mathrm{H}^{+}$ & $n=1 \ldots 3$ & 9 \\
\hline 222,223 & $\mathrm{Fe}^{n+}+\mathrm{H}^{+} \rightarrow \mathrm{Fe}^{(n+1)+}+\mathrm{H}$ & $n=0,1$ & 9 \\
\hline 224,225 & $\mathrm{Fe}^{(n+1)+}+\mathrm{He} \rightarrow \mathrm{Fe}^{n+}+\mathrm{He}^{+}$ & $n=2,3$ & 9 \\
\hline 300 & $\mathrm{H}+\mathrm{e}^{-} \rightarrow \mathrm{H}^{-}$ & & 12 \\
\hline 301 & $\mathrm{H}^{-}+\mathrm{H} \rightarrow \mathrm{H}_{2}+\mathrm{e}^{-}$ & & 13 \\
\hline 302 & $\mathrm{H}+\mathrm{H}^{+} \rightarrow \mathrm{H}_{2}^{+}$ & & 14 \\
\hline 303 & $\mathrm{H}+\mathrm{H}_{2}^{+} \rightarrow \mathrm{H}_{2}^{2}+\mathrm{H}^{+}$ & & 15 \\
\hline 304 & $\mathrm{H}^{+}+\mathrm{H}_{2} \rightarrow \mathrm{H}_{2}^{+}+\mathrm{H}$ & & 16 \\
\hline 305 & $\mathrm{H}_{2}+\mathrm{e}^{-} \rightarrow 2 \mathrm{H}+\mathrm{e}^{-}$ & & 17 \\
\hline 306 & $\mathrm{H}_{2}+\mathrm{H} \rightarrow 3 \mathrm{H}$ & & 18,19 \\
\hline 307 & $\mathrm{H}^{-}+\mathrm{e}^{-} \rightarrow \mathrm{H}+2 \mathrm{e}^{-}$ & & 1 \\
\hline 308 & $\mathrm{H}^{-}+\mathrm{H} \rightarrow 2 \mathrm{H}+\mathrm{e}^{-}$ & & 1 \\
\hline 309 & $\mathrm{H}^{-}+\mathrm{H}^{+} \rightarrow 2 \mathrm{H}$ & & 20 \\
\hline 310 & $\mathrm{H}^{-}+\mathrm{H}^{+} \rightarrow \mathrm{H}_{2}^{+}+\mathrm{e}^{-}$ & & 21 \\
\hline 311 & $\mathrm{H}_{2}^{+}+\mathrm{e}^{-} \rightarrow 2 \mathrm{H}$ & & 22 \\
\hline 312 & $\mathrm{H}_{2}^{+}+\mathrm{H}^{-} \rightarrow \mathrm{H}+\mathrm{H}_{2}$ & & 23 \\
\hline 313 & $3 \stackrel{\mathrm{H}}{\mathrm{H}} \rightarrow \mathrm{H}_{2}+\mathrm{H}$ & & 24,25 \\
\hline 314 & $2 \mathrm{H}+\mathrm{H}_{2} \rightarrow 2 \mathrm{H}_{2}$ & & 26 \\
\hline 315 & $2 \mathrm{H}_{2} \rightarrow \mathrm{H}_{2}+2 \mathrm{H}$ & & 27,28 \\
\hline 316 & $\mathrm{He}^{+}+\mathrm{H}^{-} \rightarrow \mathrm{He}+\mathrm{H}$ & & 29 \\
\hline 317 & $2 \mathrm{H}+\mathrm{He} \rightarrow \mathrm{H}_{2}+\mathrm{He}$ & & 30 \\
\hline 318 & $\mathrm{H}_{2}+\mathrm{He} \rightarrow 2 \mathrm{H}+\mathrm{He}$ & & 31 \\
\hline 319 & $\mathrm{H}_{2}+\mathrm{He}^{+} \rightarrow \mathrm{H}_{2}^{+}+\mathrm{He}$ & & 32 \\
\hline
\end{tabular}


Table A.1. continued.

\begin{tabular}{|c|c|c|c|}
\hline No. & Reaction & Notes & Ref. \\
\hline 320 & $\mathrm{H}_{2}+\mathrm{He}^{+} \rightarrow \mathrm{H}^{+}+\mathrm{H}+\mathrm{He}$ & & 32 \\
\hline 321 & $\mathrm{H}_{2}+\mathrm{H}^{+} \rightarrow \mathrm{H}_{3}^{+}$ & & 33 \\
\hline 322 & $\mathrm{H}_{3}^{+}+\mathrm{e}^{-} \rightarrow \mathrm{H}_{2}^{3}+\mathrm{H}$ & & 34 \\
\hline 323 & $\mathrm{H}_{3}^{+}+\mathrm{e}^{-} \rightarrow 3 \mathrm{H}$ & & 34 \\
\hline 324 & $\mathrm{H}_{2}^{+}+\mathrm{H}_{2} \rightarrow \mathrm{H}_{3}^{+}+\mathrm{H}$ & & 35 \\
\hline 325 & $\mathrm{H}_{3}^{+}+\mathrm{H} \rightarrow \mathrm{H}_{2}^{+}+\mathrm{H}_{2}$ & & 36 \\
\hline 326 & $\mathrm{H}_{2}^{+}+\mathrm{H}^{-} \rightarrow 3 \mathrm{H}$ & & 23 \\
\hline 327 & $\mathrm{H}_{2}^{2}+\mathrm{e}^{-} \rightarrow \mathrm{H}+\mathrm{H}^{-}$ & & 37 \\
\hline 328 & $\mathrm{He}+\mathrm{H}^{-} \rightarrow \mathrm{He}+\mathrm{H}+\mathrm{e}^{-}$ & & 38 \\
\hline 350 & $\mathrm{D}+\mathrm{e}^{-} \rightarrow \mathrm{D}^{+}+2 \mathrm{e}^{-}$ & & 1 \\
\hline 351 & $\mathrm{D}^{+}+\mathrm{e}^{-} \rightarrow \mathrm{D}$ & & 2 \\
\hline 352 & $\mathrm{D}+\mathrm{He}^{+} \rightarrow \mathrm{D}^{+}+\mathrm{He}$ & & 5 \\
\hline 353 & $\mathrm{D}^{+}+\mathrm{He} \rightarrow \mathrm{D}+\mathrm{He}^{+}$ & & 6 \\
\hline 354 & $\mathrm{D}^{+}+\mathrm{H} \rightarrow \mathrm{D}+\mathrm{H}^{+}$ & & 39 \\
\hline 355 & $\mathrm{D}+\mathrm{H}^{+} \rightarrow \mathrm{D}^{+}+\mathrm{H}$ & & 39 \\
\hline 356 & $\mathrm{D}^{+}+\mathrm{H}_{2} \rightarrow \mathrm{HD}+\mathrm{H}^{+}$ & & 40 \\
\hline 357 & $\mathrm{HD}+\mathrm{H}^{+} \rightarrow \mathrm{H}_{2}+\mathrm{D}^{+}$ & & 40 \\
\hline 358 & $\mathrm{H}_{2}+\mathrm{D} \rightarrow \mathrm{HD}+\mathrm{H}$ & & 41,42 \\
\hline 359 & $\mathrm{HD}+\mathrm{H} \rightarrow \mathrm{D}+\mathrm{H}_{2}$ & & 43 \\
\hline 400 & $\mathrm{C}^{-}+\mathrm{H}^{+} \rightarrow \mathrm{C}+\mathrm{H}$ & & 29 \\
\hline 401 & $\mathrm{C}^{-}+\mathrm{H} \rightarrow \mathrm{CH}+\mathrm{e}^{-}$ & & 29 \\
\hline 402 & $\mathrm{C}^{-}+\mathrm{H}_{2} \rightarrow \mathrm{CH}_{2}+\mathrm{e}^{-}$ & & 29 \\
\hline 403 & $\mathrm{C}^{-}+\mathrm{O} \rightarrow \mathrm{CO}+\mathrm{e}^{-}$ & & 29 \\
\hline 404 & $\mathrm{O}^{-}+\mathrm{H}^{+} \rightarrow \mathrm{O}+\mathrm{H}$ & & 29 \\
\hline 405 & $\mathrm{O}^{-}+\mathrm{H} \rightarrow \mathrm{OH}+\mathrm{e}^{-}$ & & 29 \\
\hline 406 & $\mathrm{O}^{-}+\mathrm{H}_{2} \rightarrow \mathrm{H}_{2} \mathrm{O}+\mathrm{e}^{-}$ & & 29 \\
\hline 407 & $\mathrm{O}^{-}+\mathrm{C} \rightarrow \mathrm{CO}+\mathrm{e}^{-}$ & & 29 \\
\hline 408 & $\mathrm{C}+\mathrm{e}^{-} \rightarrow \mathrm{C}^{-}$ & & 44 \\
\hline 409 & $\mathrm{C}+\mathrm{H}^{-} \rightarrow \mathrm{CH}+\mathrm{e}^{-}$ & & 29 \\
\hline 410 & $\mathrm{C}+\mathrm{H} \rightarrow \mathrm{CH}$ & & 45 \\
\hline 411 & $\mathrm{C}+\mathrm{H}_{2} \rightarrow \mathrm{CH}_{2}$ & & 45 \\
\hline 412 & $\mathrm{C}+\mathrm{H}_{2} \rightarrow \mathrm{CH}+\mathrm{H}$ & & 46 \\
\hline 413 & $\mathrm{C}+\mathrm{H}_{2}^{+} \rightarrow \mathrm{CH}^{+}+\mathrm{H}$ & & 29 \\
\hline 414 & $\mathrm{C}+\mathrm{H}_{3}^{+} \rightarrow \mathrm{CH}^{+}+\mathrm{H}_{2}$ & & 29 \\
\hline 415 & $2 \mathrm{C} \rightarrow \mathrm{C}_{2}$ & & 47 \\
\hline 416 & $\mathrm{C}+\mathrm{O} \rightarrow \mathrm{CO}$ & & 48,49 \\
\hline 417 & $\mathrm{C}^{+}+\mathrm{H} \rightarrow \mathrm{CH}^{+}$ & & 50 \\
\hline 418 & $\mathrm{C}^{+}+\mathrm{H}_{2} \rightarrow \mathrm{CH}^{+}+\mathrm{H}$ & & 51 \\
\hline 419 & $\mathrm{C}^{+}+\mathrm{H}_{2} \rightarrow \mathrm{CH}_{2}^{+}$ & & 29 \\
\hline 420 & $\mathrm{C}^{+}+\mathrm{O} \rightarrow \mathrm{CO}^{+2}$ & & 48 \\
\hline 421 & $\mathrm{O}+\mathrm{e}^{-} \rightarrow \mathrm{O}^{-}$ & & 29 \\
\hline 422 & $\mathrm{O}+\mathrm{H}^{-} \rightarrow \mathrm{OH}+\mathrm{e}^{-}$ & & 29 \\
\hline 423 & $\mathrm{O}+\mathrm{H} \rightarrow \mathrm{OH}$ & & 29 \\
\hline 424 & $\mathrm{O}+\mathrm{H}_{2} \rightarrow \mathrm{OH}+\mathrm{H}$ & & 52 \\
\hline 425 & $\mathrm{O}+\mathrm{H}_{2}^{+} \rightarrow \mathrm{OH}^{+}+\mathrm{H}$ & & 29 \\
\hline 426 & $\mathrm{O}+\mathrm{H}_{3}^{+} \rightarrow \mathrm{OH}^{+}+\mathrm{H}_{2}$ & & 53 \\
\hline 427 & $2 \mathrm{O} \rightarrow \mathrm{O}_{2}$ & & 45 \\
\hline 428 & $\mathrm{O}^{+}+\mathrm{H}_{2} \rightarrow \mathrm{OH}^{+}+\mathrm{H}$ & & 54,55 \\
\hline 429 & $\mathrm{C}_{2}+\mathrm{He}^{+} \rightarrow \mathrm{C}^{+}+\mathrm{C}+\mathrm{He}$ & & 29 \\
\hline 430 & $\mathrm{C}_{2}+\mathrm{O} \rightarrow \mathrm{CO}+\mathrm{C}$ & & 56,57 \\
\hline 431 & $\mathrm{C}_{2}+\mathrm{O}^{+} \rightarrow \mathrm{CO}^{+}+\mathrm{C}$ & & 29 \\
\hline 432 & $\mathrm{O}_{2}+\mathrm{H} \rightarrow \mathrm{OH}+\mathrm{O}$ & & 29 \\
\hline 433 & $\mathrm{O}_{2}+\mathrm{H}^{+} \rightarrow \mathrm{O}_{2}^{+}+\mathrm{H}$ & & 58 \\
\hline 434 & $\mathrm{O}_{2}+\mathrm{He}^{+} \rightarrow \mathrm{O}_{2}^{+}+\mathrm{He}$ & & 59,60 \\
\hline 435 & $\mathrm{O}_{2}+\mathrm{He}^{+} \rightarrow \mathrm{O}^{2}+\mathrm{O}+\mathrm{He}$ & & 59,60 \\
\hline 436 & $\mathrm{O}_{2}+\mathrm{H}_{2} \rightarrow 2 \mathrm{OH}$ & & 61 \\
\hline 437 & $\mathrm{O}_{2}+\mathrm{C} \rightarrow \mathrm{CO}+\mathrm{O}$ & & 62 \\
\hline 438 & $\mathrm{O}_{2}+\mathrm{C}^{+} \rightarrow \mathrm{CO}+\mathrm{O}^{+}$ & & 63,64 \\
\hline
\end{tabular}

Table A.1. continued.

\begin{tabular}{|c|c|c|c|}
\hline No. & Reaction & Notes & Ref. \\
\hline 439 & $\mathrm{O}_{2}+\mathrm{C}^{+} \rightarrow \mathrm{CO}^{+}+\mathrm{O}$ & & 63,64 \\
\hline 440 & $\mathrm{O}_{2}+\mathrm{CH}_{2}^{+} \rightarrow \mathrm{HCO}^{+}+\mathrm{OH}$ & & 63,64 \\
\hline 441 & $\mathrm{O}_{2}^{+}+\mathrm{e}^{-} \stackrel{2}{\rightarrow} 2 \mathrm{O}$ & & 65 \\
\hline 442 & $\mathrm{O}_{2}^{+}+\mathrm{C} \rightarrow \mathrm{O}_{2}+\mathrm{C}^{+}$ & & 29 \\
\hline 443 & $\mathrm{O}_{2}^{+}+\mathrm{C} \rightarrow \mathrm{CO}^{+}+\mathrm{O}$ & & 29 \\
\hline 444 & $\mathrm{CH}+\mathrm{H} \rightarrow \mathrm{C}+\mathrm{H}_{2}$ & & 66 \\
\hline 445 & $\mathrm{CH}+\mathrm{H}_{2} \rightarrow \mathrm{CH}_{2}+\mathrm{H}$ & & 29 \\
\hline 446 & $\mathrm{CH}+\mathrm{C} \rightarrow \mathrm{C}_{2}+\mathrm{H}$ & & 62 \\
\hline 447 & $\mathrm{CH}+\mathrm{O} \rightarrow \mathrm{CO}+\mathrm{H}$ & & 67,68 \\
\hline 448 & $\mathrm{CH}+\mathrm{O} \rightarrow \mathrm{HCO}^{+}+\mathrm{e}^{-}$ & & 69 \\
\hline 449 & $\mathrm{CH}+\mathrm{H}^{+} \rightarrow \mathrm{CH}^{+}+\mathrm{H}$ & & 29 \\
\hline 450 & $\mathrm{CH}^{+}+\mathrm{e}^{-} \rightarrow \mathrm{C}+\mathrm{H}$ & & 70 \\
\hline 451 & $\mathrm{CH}^{+}+\mathrm{H} \rightarrow \mathrm{C}^{+}+\mathrm{H}_{2}$ & & 71 \\
\hline 452 & $\mathrm{CH}^{+}+\mathrm{H}_{2} \rightarrow \mathrm{CH}_{2}^{+}+\mathrm{H}$ & & 71 \\
\hline 453 & $\mathrm{CH}^{+}+\mathrm{O} \rightarrow \mathrm{CO}^{+}+\mathrm{H}$ & & 72 \\
\hline 454 & $\mathrm{CH}_{2}+\mathrm{H} \rightarrow \mathrm{CH}+\mathrm{H}_{2}$ & & 29 \\
\hline 455 & $\mathrm{CH}_{2}+\mathrm{O} \rightarrow \mathrm{CO}+2 \mathrm{H}$ & & 73 \\
\hline 456 & $\mathrm{CH}_{2}+\mathrm{O} \rightarrow \mathrm{CO}+\mathrm{H}_{2}$ & & 29 \\
\hline 457 & $\mathrm{CH}_{2}+\mathrm{H}^{+} \rightarrow \mathrm{CH}^{+}+\mathrm{H}_{2}$ & & 29 \\
\hline 458 & $\mathrm{CH}_{2}+\mathrm{H}^{+} \rightarrow \mathrm{CH}_{2}^{+}+\mathrm{H}$ & & 29 \\
\hline 459 & $\mathrm{CH}_{2}+\mathrm{He}^{+} \rightarrow \mathrm{C}^{+2}+\mathrm{H}_{2}+\mathrm{He}$ & & 29 \\
\hline 460 & $\mathrm{CH}_{2}^{+}+\mathrm{e}^{-} \rightarrow \mathrm{CH}+\mathrm{H}$ & & 74 \\
\hline 461 & $\mathrm{CH}_{2}^{+}+\mathrm{e}^{-} \rightarrow \mathrm{C}+2 \mathrm{H}$ & & 74 \\
\hline 462 & $\mathrm{CH}_{2}^{+}+\mathrm{e}^{-} \rightarrow \mathrm{C}+\mathrm{H}_{2}$ & & 74 \\
\hline 463 & $\mathrm{CH}_{2}^{+}+\mathrm{H} \rightarrow \mathrm{CH}^{+}+\mathrm{H}_{2}$ & & 29 \\
\hline 464 & $\mathrm{CH}_{2}^{+}+\mathrm{H}_{2} \rightarrow \mathrm{CH}_{3}^{+}+\mathrm{H}$ & & 63,64 \\
\hline 465 & $\mathrm{CH}_{2}^{+}+\mathrm{O} \rightarrow \mathrm{HCO}^{+}+\mathrm{H}$ & & 45 \\
\hline 466 & $\mathrm{CH}_{3}^{+}+\mathrm{e}^{-} \rightarrow \mathrm{CH}_{2}+\mathrm{H}$ & & 75 \\
\hline 467 & $\mathrm{CH}_{3}^{+}+\mathrm{e}^{-} \rightarrow \mathrm{CH}+\mathrm{H}_{2}$ & & 75 \\
\hline 468 & $\mathrm{CH}_{3}^{+}+\mathrm{e}^{-} \rightarrow \mathrm{CH}+2 \mathrm{H}$ & & 29 \\
\hline 469 & $\mathrm{CH}_{3}^{+}+\mathrm{H} \rightarrow \mathrm{CH}_{2}^{+}+\mathrm{H}_{2}$ & & 29 \\
\hline 470 & $\mathrm{CH}_{3}^{+}+\mathrm{O} \rightarrow \mathrm{HCO}^{+}+\mathrm{H}_{2}$ & & 76 \\
\hline 471 & $\mathrm{OH}^{3}+\mathrm{H}^{-} \rightarrow \mathrm{H}_{2} \mathrm{O}+\mathrm{e}^{-}$ & & 29 \\
\hline 472 & $\mathrm{OH}+\mathrm{H} \rightarrow \mathrm{O}+2 \mathrm{H}$ & & 29 \\
\hline 473 & $\mathrm{OH}+\mathrm{H} \rightarrow \mathrm{H}_{2} \mathrm{O}$ & & 77 \\
\hline 474 & $\mathrm{OH}+\mathrm{H} \rightarrow \mathrm{O}+\mathrm{H}_{2}$ & & 78 \\
\hline 475 & $\mathrm{OH}+\mathrm{H}_{2} \rightarrow \mathrm{H}_{2} \mathrm{O}+\mathrm{H}$ & & 79 \\
\hline 476 & $\mathrm{OH}+\mathrm{C} \rightarrow \mathrm{CO}+\mathrm{H}$ & & 62 \\
\hline 477 & $\mathrm{OH}+\mathrm{O} \rightarrow \mathrm{O}_{2}+\mathrm{H}$ & & 29 \\
\hline 478 & $2 \mathrm{OH} \rightarrow \mathrm{O}+\mathrm{H}_{2} \mathrm{O}$ & & 62 \\
\hline 479 & $\mathrm{OH}+\mathrm{H}^{+} \rightarrow \mathrm{OH}^{+}+\mathrm{H}$ & & 29 \\
\hline 480 & $\mathrm{OH}+\mathrm{He}^{+} \rightarrow \mathrm{O}^{+}+\mathrm{H}+\mathrm{He}$ & & 29 \\
\hline 481 & $\mathrm{OH}+\mathrm{H}_{3}^{+} \rightarrow \mathrm{H}_{2} \mathrm{O}^{+}+\mathrm{H}_{2}$ & & 29 \\
\hline 482 & $\mathrm{OH}+\mathrm{C}^{+} \rightarrow \mathrm{CO}^{+}+\mathrm{H}$ & & 80 \\
\hline 483 & $\mathrm{OH}+\mathrm{C}^{+} \rightarrow \mathrm{CO}+\mathrm{H}^{+}$ & & 80 \\
\hline 484 & $\mathrm{OH}^{+}+\mathrm{e}^{-} \rightarrow \mathrm{O}+\mathrm{H}$ & & 81 \\
\hline 485 & $\mathrm{OH}^{+}+\mathrm{H}_{2} \rightarrow \mathrm{H}_{2} \mathrm{O}^{+}+\mathrm{H}$ & & 82 \\
\hline 486 & $\mathrm{H}_{2} \mathrm{O}+\mathrm{H} \rightarrow \mathrm{OH}+\mathrm{H}_{2}$ & & 83 \\
\hline 487 & $\mathrm{H}_{2} \mathrm{O}+\mathrm{H}^{+} \rightarrow \mathrm{H}_{2} \mathrm{O}^{+}+\mathrm{H}$ & & 58 \\
\hline 488 & $\mathrm{H}_{2} \mathrm{O}+\mathrm{He}^{+} \rightarrow \mathrm{OH}+\mathrm{H}^{+}+\mathrm{He}$ & & 84,85 \\
\hline 489 & $\mathrm{H}_{2} \mathrm{O}+\mathrm{He}^{+} \rightarrow \mathrm{OH}^{+}+\mathrm{H}+\mathrm{He}$ & & 84,85 \\
\hline 490 & $\mathrm{H}_{2} \mathrm{O}+\mathrm{He}^{+} \rightarrow \mathrm{H}_{2} \mathrm{O}^{+}+\mathrm{He}$ & & 84,85 \\
\hline 491 & $\mathrm{H}_{2} \mathrm{O}+\mathrm{H}_{3}^{+} \rightarrow \mathrm{H}_{3} \mathrm{O}^{+}+\mathrm{H}_{2}$ & & 86,87 \\
\hline 492 & $\mathrm{H}_{2} \mathrm{O}+\mathrm{C}^{+} \rightarrow \mathrm{HCO}^{+}+\mathrm{H}$ & & 88,89 \\
\hline 493 & $\mathrm{H}_{2} \mathrm{O}+\mathrm{C}^{+} \rightarrow \mathrm{HOC}^{+}+\mathrm{H}$ & & 90 \\
\hline 494 & $\mathrm{H}_{2} \mathrm{O}^{+}+\mathrm{e}^{-} \rightarrow \mathrm{O}+2 \mathrm{H}$ & & 91 \\
\hline 495 & $\mathrm{H}_{2} \mathrm{O}^{+}+\mathrm{e}^{-} \rightarrow \mathrm{O}+\mathrm{H}_{2}$ & & 91 \\
\hline 496 & $\mathrm{H}_{2} \mathrm{O}^{+}+\mathrm{e}^{-} \rightarrow \mathrm{OH}+\mathrm{H}$ & & 91 \\
\hline
\end{tabular}


Table A.1. continued.

\begin{tabular}{|c|c|c|c|}
\hline No. & Reaction & Notes & Ref. \\
\hline 497 & $\mathrm{H}_{2} \mathrm{O}^{+}+\mathrm{H}_{2} \rightarrow \mathrm{H}_{3} \mathrm{O}^{+}+\mathrm{H}$ & & 92 \\
\hline 498 & $\mathrm{H}_{3} \mathrm{O}^{+}+\mathrm{e}^{-} \rightarrow \mathrm{H}_{2} \mathrm{O}+\mathrm{H}$ & & 93 \\
\hline 499 & $\mathrm{H}_{3} \mathrm{O}^{+}+\mathrm{e}^{-} \rightarrow \mathrm{OH}+\mathrm{H}_{2}$ & & 93 \\
\hline 500 & $\mathrm{H}_{3} \mathrm{O}^{+}+\mathrm{e}^{-} \rightarrow \mathrm{OH}+2 \mathrm{H}$ & & 93 \\
\hline 501 & $\mathrm{H}_{3} \mathrm{O}^{+}+\mathrm{e}^{-} \rightarrow \mathrm{O}+\mathrm{H}+\mathrm{H}_{2}$ & & 94 \\
\hline 502 & $\mathrm{H}_{3} \mathrm{O}^{+}+\mathrm{C} \rightarrow \mathrm{HCO}^{+}+\mathrm{H}_{2}$ & & 45 \\
\hline 503 & $\mathrm{CO}+\mathrm{H} \rightarrow \mathrm{C}+\mathrm{OH}$ & & 95 \\
\hline 504 & $\mathrm{CO}+\mathrm{H}_{3}^{+} \rightarrow \mathrm{HOC}^{+}+\mathrm{H}_{2}$ & & 96 \\
\hline 505 & $\mathrm{CO}+\mathrm{H}_{3}^{+} \rightarrow \mathrm{HCO}^{+}+\mathrm{H}_{2}$ & & 96 \\
\hline 506 & $\mathrm{CO}+\mathrm{He}^{+} \rightarrow \mathrm{C}^{+}+\mathrm{O}+\mathrm{He}$ & & 97 \\
\hline 507 & $\mathrm{CO}+\mathrm{He}^{+} \rightarrow \mathrm{O}^{+}+\mathrm{C}+\mathrm{He}$ & & 97 \\
\hline 508 & $\mathrm{CO}^{+}+\mathrm{e}^{-} \rightarrow \mathrm{C}+\mathrm{O}$ & & 98 \\
\hline 509 & $\mathrm{CO}^{+}+\mathrm{H} \rightarrow \mathrm{CO}+\mathrm{H}^{+}$ & & 99 \\
\hline 510 & $\mathrm{CO}^{+}+\mathrm{H}_{2} \rightarrow \mathrm{HCO}^{+}+\mathrm{H}$ & & 100 \\
\hline 511 & $\mathrm{CO}^{+}+\mathrm{H}_{2} \rightarrow \mathrm{HOC}^{+}+\mathrm{H}$ & & 100 \\
\hline 512 & $\mathrm{HCO}^{+}+\mathrm{e}^{-} \rightarrow \mathrm{OH}+\mathrm{C}$ & & 101 \\
\hline 513 & $\mathrm{HCO}^{+}+\mathrm{e}^{-} \rightarrow \mathrm{CO}+\mathrm{H}$ & & 101 \\
\hline 514 & $\mathrm{HCO}^{+}+\mathrm{C} \rightarrow \mathrm{CO}+\mathrm{CH}^{+}$ & & 45 \\
\hline 515 & $\mathrm{HCO}^{+}+\mathrm{H}_{2} \mathrm{O} \rightarrow \mathrm{CO}+\mathrm{H}_{3} \mathrm{O}^{+}$ & & 102 \\
\hline 516 & $\mathrm{HOC}^{+}+\mathrm{e}^{-} \rightarrow \mathrm{CO}+\mathrm{H}$ & & 29 \\
\hline 517 & $\mathrm{HOC}^{+}+\mathrm{H}_{2} \rightarrow \mathrm{HCO}^{+}+\mathrm{H}_{2}$ & & 103 \\
\hline 518 & $\mathrm{HOC}^{+}+\mathrm{CO} \rightarrow \mathrm{HCO}^{+}+\mathrm{CO}$ & & 104 \\
\hline 519 & $2 \mathrm{C}+\mathrm{He} \rightarrow \mathrm{C}_{2}+\mathrm{He}$ & & 105 \\
\hline 520 & $2 \mathrm{O}+\mathrm{He} \rightarrow \mathrm{O}_{2}+\mathrm{He}$ & & 106 \\
\hline 521 & $\mathrm{C}+\mathrm{O}+\mathrm{He} \rightarrow \mathrm{CO}+\mathrm{He}$ & & 97 \\
\hline 522 & $\mathrm{C}^{+}+\mathrm{O}+\mathrm{He} \rightarrow \mathrm{CO}^{+}+\mathrm{He}$ & & 97 \\
\hline 523 & $\mathrm{O}^{+}+\mathrm{C}+\mathrm{He} \rightarrow \mathrm{CO}^{+}+\mathrm{He}$ & & 97 \\
\hline 524 & $\mathrm{O}+\mathrm{H}+\mathrm{He} \rightarrow \mathrm{OH}+\mathrm{He}$ & & 78 \\
\hline 525 & $\mathrm{OH}+\mathrm{H}+\mathrm{He} \rightarrow \mathrm{H}_{2} \mathrm{O}+\mathrm{He}$ & & 67 \\
\hline
\end{tabular}

Table A.2. $\mathrm{NC}^{2} \mathrm{M}$ - CR-induced reactions.

\begin{tabular}{|c|c|c|c|c|}
\hline No. & Reaction & \multicolumn{2}{|l|}{$\zeta^{\mathrm{rel}}$} & Ref. \\
\hline 600 & $\mathrm{H} \stackrel{\text { cr }}{\rightarrow} \mathrm{H}^{+}+\mathrm{e}^{-}$ & \multicolumn{2}{|l|}{1.0} & 1 \\
\hline 601 & $\mathrm{D} \stackrel{\text { cr }}{\rightarrow} \mathrm{D}^{+}+\mathrm{e}^{-}$ & \multicolumn{2}{|l|}{1.0} & 1 \\
\hline 602 & $\mathrm{He} \stackrel{\text { cr }}{\rightarrow} \mathrm{He}^{+}+\mathrm{e}^{-}$ & \multicolumn{2}{|l|}{1.1} & 2 \\
\hline 603 & $\mathrm{C} \stackrel{\text { cr }}{\rightarrow} \mathrm{C}^{+}+\mathrm{e}^{-}$ & \multicolumn{2}{|l|}{3.8} & 2 \\
\hline 604 & $\mathrm{C}^{+} \stackrel{\text { cr }}{\rightarrow} \mathrm{C}^{++}+\mathrm{e}^{-}$ & \multicolumn{2}{|l|}{1.7} & 3,4 \\
\hline 605 & $\mathrm{~N} \stackrel{\text { cr }}{\rightarrow} \mathrm{N}^{+}+\mathrm{e}^{-}$ & \multicolumn{2}{|l|}{4.6} & 2 \\
\hline 606 & $\mathrm{~N}^{+} \stackrel{\mathrm{cr}}{\rightarrow} \mathrm{N}^{++}+\mathrm{e}^{-}$ & \multicolumn{2}{|l|}{1.8} & 3,4 \\
\hline 607 & $\mathrm{O} \stackrel{\text { cr }}{\rightarrow} \mathrm{O}^{+}+\mathrm{e}^{-}$ & \multicolumn{2}{|l|}{5.7} & 2 \\
\hline 608 & $\mathrm{O}^{+} \stackrel{\text { cr }}{\rightarrow} \mathrm{O}^{++}+\mathrm{e}^{-}$ & \multicolumn{2}{|l|}{1.9} & 3,4 \\
\hline 609 & $\mathrm{Ne} \stackrel{\text { cr }}{\rightarrow} \mathrm{Ne}^{+}+\mathrm{e}^{-}$ & \multicolumn{2}{|l|}{4.8} & 3,4 \\
\hline 610 & $\mathrm{Ne}^{+} \stackrel{\text { cr }}{\rightarrow} \mathrm{Ne}^{++}+\mathrm{e}^{-}$ & \multicolumn{2}{|l|}{2.3} & 3,4 \\
\hline 613 & $\mathrm{Si} \stackrel{\text { cr }}{\rightarrow} \mathrm{Si}^{+}+\mathrm{e}^{-}$ & \multicolumn{2}{|l|}{6.5} & 3,4 \\
\hline 614 & $\mathrm{Si}^{+} \stackrel{\text { cr }}{\rightarrow} \mathrm{Si}^{++}+\mathrm{e}^{-}$ & \multicolumn{2}{|l|}{2.5} & 3,4 \\
\hline 615 & $\mathrm{H}_{2} \stackrel{\mathrm{cr}}{\rightarrow} \mathrm{H}_{2}^{+}+\mathrm{e}^{-}$ & \multicolumn{2}{|l|}{2.0} & 2 \\
\hline 616 & $\mathrm{CO} \stackrel{\text { cr }}{\rightarrow} \mathrm{CO}^{+}+\mathrm{e}^{-}$ & \multicolumn{2}{|l|}{6.5} & 2 \\
\hline \multirow[t]{2}{*}{617} & $\mathrm{H}_{2} \stackrel{\text { cr }}{\rightarrow} 2 \mathrm{H}$ & \multicolumn{2}{|l|}{0.22} & 2 \\
\hline & & $q_{\mathrm{X}}$ & $p_{\mathrm{X}}$ & \\
\hline 650 & $\mathrm{C} \stackrel{\gamma_{\text {cr }}}{\rightarrow} \mathrm{C}^{+}+\mathrm{e}^{-}$ & 0 & 510 & 5,6 \\
\hline 651 & $\mathrm{Si} \stackrel{\gamma_{\mathrm{cr}}}{\rightarrow} \mathrm{Si}^{+}+\mathrm{e}^{-}$ & 14000 & 8350 & 5,6 \\
\hline 652 & $\mathrm{Fe} \stackrel{\gamma_{\mathrm{cr}}}{\rightarrow} \mathrm{Fe}^{+}+\mathrm{e}^{-}$ & 300 & 447 & 5,6 \\
\hline 653 & $\mathrm{C}_{2} \stackrel{\gamma_{\mathrm{cr}}}{\rightarrow} 2 \mathrm{C}$ & - & 237 & 5 \\
\hline 654 & $\mathrm{O}_{2} \stackrel{\gamma_{\mathrm{cr}}}{\rightarrow} 2 \mathrm{O}$ & 750 & 751 & 5,6 \\
\hline 655 & $\mathrm{O}_{2} \stackrel{\gamma_{\mathrm{cr}}}{\rightarrow} \mathrm{O}_{2}^{+}+\mathrm{e}^{-}$ & - & 117 & 5 \\
\hline 656 & $\mathrm{CH} \stackrel{\gamma_{\mathrm{cr}}}{\rightarrow} \mathrm{C}+\mathrm{H}$ & 350 & 730 & 5,6 \\
\hline 657 & $\mathrm{CH}^{+} \stackrel{\gamma_{\mathrm{cr}}}{\rightarrow} \mathrm{C}+\mathrm{H}^{+}$ & - & 176 & 5 \\
\hline 658 & $\mathrm{CH}_{2} \stackrel{\gamma_{\text {cr }}}{\rightarrow} \mathrm{CH}+\mathrm{H}$ & - & 250 & 5,2 \\
\hline 659 & $\mathrm{CH}_{2} \stackrel{\gamma_{\text {cr }}}{\rightarrow} \mathrm{CH}_{2}^{+}+\mathrm{e}^{-}$ & - & 250 & 5,2 \\
\hline 660 & $\mathrm{OH} \stackrel{\gamma_{\mathrm{cr}}}{\rightarrow} \mathrm{O}+\mathrm{H}$ & 1000 & 509 & 5,6 \\
\hline 661 & $\mathrm{H}_{2} \mathrm{O} \stackrel{\gamma_{\mathrm{cr}}}{\rightarrow} \mathrm{OH}+\mathrm{H}$ & 3700 & 971 & 5,6 \\
\hline 662 & $\mathrm{CO} \stackrel{\gamma_{\mathrm{cr}}}{\rightarrow} \mathrm{C}+\mathrm{O}$ & $\mathrm{Se}$ & & 7 \\
\hline
\end{tabular}

References. 1: Williams et al. (1998), 2: McElroy et al. (2013), 3: Langer (1978), 4: Lotz (1967), 5: Gredel et al. (1989), 6: Maloney et al. (1996) and 7: Gredel et al. (1987).

Table A.3. $\mathrm{NC}^{2} \mathrm{M}$ - dust-catalytic reactions.

\begin{tabular}{lll}
\hline \hline No. & Reaction $^{a}$ & Ref. \\
\hline 700 & $2 \mathrm{H}(+$ grain $) \stackrel{\text { dust }}{\rightarrow} \mathrm{H}_{2}(+$ grain $)$ & See Sect. 2.3 \\
701 & $\mathrm{H}^{+}(+$grain $) \stackrel{\text { dust }}{\rightarrow} \mathrm{H}\left(+\right.$ grain $\left.^{+}\right)$ & See Sect. 2.3 \\
702 & $\mathrm{He}^{+}(+$grain $) \stackrel{\text { dust }}{\rightarrow} \mathrm{He}\left(+\right.$ grain $\left.^{+}\right)$ & See Sect. 2.3 \\
703 & $\mathrm{C}^{+}(+$grain $) \stackrel{\text { dust }}{\rightarrow} \mathrm{C}\left(+\right.$ grain $\left.^{+}\right)$ & See Sect. 2.3 \\
704 & $\mathrm{Mg}^{+}(+$grain $) \stackrel{\text { dust }}{\rightarrow} \mathrm{Mg}\left(+\right.$ grain $\left.^{+}\right)$ & See Sect. 2.3 \\
705 & $\mathrm{Si}^{+}(+$grain $) \stackrel{\text { dust }}{\rightarrow} \mathrm{Si}\left(+\right.$ grain $\left.^{+}\right)$ & See Sect. 2.3 \\
706 & $\mathrm{Fe}^{+}(+$grain $) \stackrel{\text { dust }}{\rightarrow} \mathrm{Fe}\left(+\right.$ grain $\left.^{+}\right)$ & See Sect. 2.3 \\
\hline
\end{tabular}

Notes. ${ }^{(a)}$ grain ${ }^{+}$denotes a dust particle that has increased its charge by $+e$. 